\title{
Evaluation of sterling silver as a contacting material for the cathode chamber of the solid-oxide fuel cell
}

John Sakacsi

West Virginia University

Follow this and additional works at: https://researchrepository.wvu.edu/etd

\section{Recommended Citation}

Sakacsi, John, "Evaluation of sterling silver as a contacting material for the cathode chamber of the solidoxide fuel cell" (2006). Graduate Theses, Dissertations, and Problem Reports. 1743.

https://researchrepository.wvu.edu/etd/1743

This Thesis is protected by copyright and/or related rights. It has been brought to you by the The Research Repository @ WVU with permission from the rights-holder(s). You are free to use this Thesis in any way that is permitted by the copyright and related rights legislation that applies to your use. For other uses you must obtain permission from the rights-holder(s) directly, unless additional rights are indicated by a Creative Commons license in the record and/ or on the work itself. This Thesis has been accepted for inclusion in WVU Graduate Theses, Dissertations, and Problem Reports collection by an authorized administrator of The Research Repository @ WVU. For more information, please contact researchrepository@mail.wvu.edu. 


\title{
EVALUATION OF STERLING SILVER AS A CONTACTING MATERIAL FOR THE CATHODE CHAMBER OF THE SOLID-OXIDE FUEL CELL
}

\author{
John Sakacsi \\ Thesis submitted to the College of Engineering and Mineral Resources \\ At West Virginia University in partial fulfillment of the \\ Requirements for the degree of \\ Master of Science \\ In \\ Mechanical Engineering \\ Bruce S. Kang, Ph.D, Chair \\ Lane Wilson, Ph.D \\ Xingbo Liu, Ph.D \\ Jacky Prucz, Ph.D \\ Department of Mechanical and Aerospace Engineering
}

Morgantown, West Virginia

2006

Keywords: Solid-oxide fuel cell, Silver, Copper-oxide, Oxidation, Microstructure analysis, Scanning electron microscopy 


\begin{abstract}
Evaluation of sterling silver as contacting material in the cathode chamber of the solidoxide fuel cell
\end{abstract}

\title{
John Sakacsi
}

In recent years the operating temperature of the SOFC has been reduced to $\sim 800 \mathrm{C}$, making it possible to use silver in the fabrication of the cell stack. Silver is attractive due to its high conductivity, ductility and the fact that it does not form non-conductive scales that could reduce the performance of the fuel cell stack. This research focuses on the development and testing of candidate materials, utilizing silver, in a simulated cathode environment using a simple tube furnace and air supply. Test specimens were fabricated using simple powder metallurgy techniques or by the oxidation of specific alloys to create desired characteristics. Samples are examined using scanning electron microscopy to evaluate microstructure, as well as energy dispersive spectroscopy to evaluate material composition.

Sterling silver was chosen as the material of primary interest due to its favorable composition and low-cost. Sterling silver is a $7.5 \% \mathrm{Cu}-\mathrm{Ag}$ alloy by weight. When exposed in an oxidizing environment the copper in the alloy will oxidize forming copper-oxide which is meant to protect the silver from air exposure and reduce the rate of silver evaporation. The sterling silver samples were compared to pure silver samples in terms of evaporation rate and surface microstructure changes.

Testing showed that the sterling silver samples exhibited a higher rate of evaporation than the pure silver samples tested under the same conditions. Scanning electron microscopy showed a much higher rate of silver faceting at the surface of the pure silver samples than the sterling silver samples indicating that the evaporation rate could be influenced by the silver surface orientation. Microstructure analysis also indicated that copper-oxide may be too volatile for use as a protective oxide due to its high volatility causing particle migration and agglomeration. 


\section{ACKNOWLEDGEMENTS}

I would like to start by thanking my research advisor and committee chair, Dr. Bruce Kang, for all of his guidance and encouragement throughout my master degree, as well as Dr. Lane Wilson, for all of his help and patience throughout this project and for continuing to have faith in me, even at times when I struggled to make progress. I would also like to express my appreciation to Dr. Xingbo Liu and Dr. Jacky Prucz for serving on my committee and for all of their help in the course of both my undergraduate and graduate work at West Virginia University.

I would also like to thank Ms. Diane Berry for all of her help with my scanning electron microscopy work at NIOSH.

I would also like to thank all of my friends for making the last couple of years some of the most memorable of my life.

Finally, I would like to thank my parents for always supporting my decisions in life and allowing me to choose my own path no matter where it takes me. I appreciate all of the sacrifices you have made for me over the years. 


\section{TABLE OF CONTENTS}

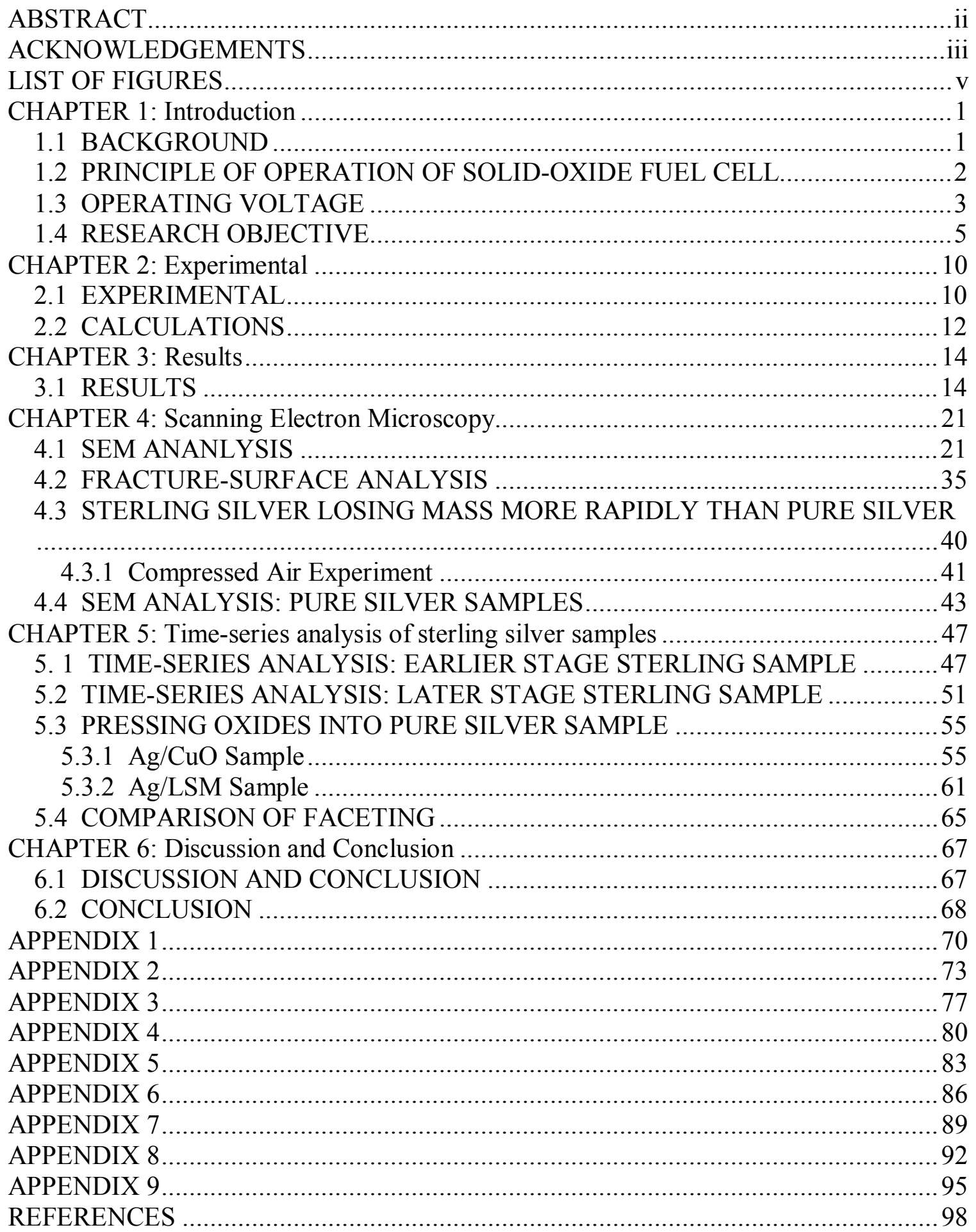




\section{LIST OF FIGURES}

Figure 1: Illustration of chemical reaction in a solid-oxide fuel cell ............................ 3

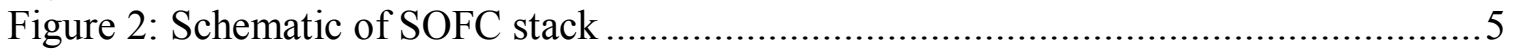

Figure 3: Schematic of contact area between interconnect and LSM cathode .................. 6

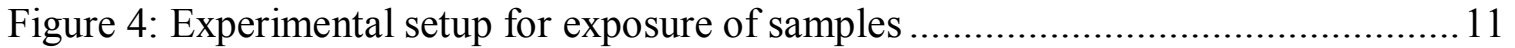

Figure 5: Sample holder for exposure experiment ................................................... 11

Figure 6: Thickness reduction of sterling silver.................................................... 14

Figure 7: Accumulating thickness reduction of sterling silver ............................... 15

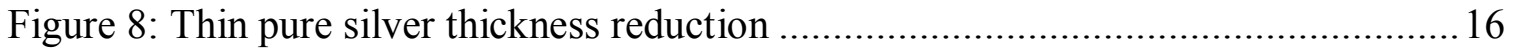

Figure 9: Accumulating thickness reduction of pure silver ....................................... 17

Figure 10: Thickness reduction of thick pure silver sample .......................................... 18

Figure 11: Accumulating thickness reduction of pure silver...................................... 19

Figure 12: Comparison of thickness reduction for all types of samples ........................ 20

Figure 13: Unexposed sterling silver surface SEM micrograph and backscatter image...21

Figure 14: EDS of sterling silver surface ........................................................22

Figure 15: Sterling silver surface and backscatter after $12 \mathrm{hrs}$ exposure with no air flow23

Figure 16: sterling silver surface and x-ray spectrum after 12 hour exposure ................23

Figure 17: Sterling silver surface and x-ray spectrum after 1-week of exposure ............24

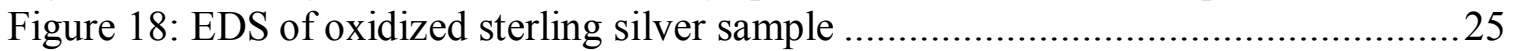

Figure 19: Sterling silver surface and x-ray spectrum after 1-day exposure..................26

Figure 20: Sterling silver surface and x-ray spectrum after 2-day exposure...................27

Figure 21: Sterling silver surface and x-ray spectrum after 3-day exposure ...................28

Figure 22: Sterling silver surface and x-ray spectrum after 1 -week exposure ................28

Figure 23: Sterling silver surface and x-ray spectrum after 2-week exposure ...............29

Figure 24: Sterling silver surface and x-ray spectrum after 4-week exposure .................30

Figure 25: Sterling silver surface and x-ray spectrum after 5-week exposure .................30

Figure 26: Sterling silver surface and x-ray spectrum after 7 -week exposure ................. 31

Figure 27: Sterling silver surface and x-ray spectrum after 10 -week exposure ................32

Figure 28: Sterling silver surface and x-ray spectrum after 13-week exposure ..............33

Figure 29: Sterling silver surface and x-ray spectrum after 17-week exposure ...............33

Figure 30: Sterling silver surface and x-ray spectrum after 27-week exposure ...............34

Figure 31: SEM micrograph and backscatter of sterling silver cross-section (1-week

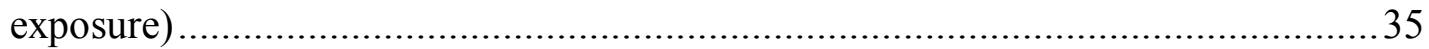

Figure 32: SEM micrograph and x-ray spectrum of sterling silver cross-section (1-week

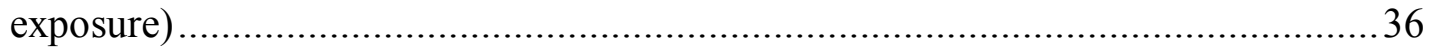

Figure 33: SEM micrograph and backscatter of sterling silver cross-section (2-week

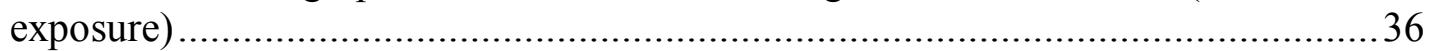

Figure 34: SEM micrograph and backscatter of sterling silver cross-section (4-week

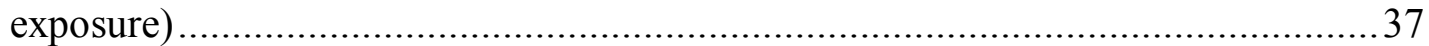

Figure 35: SEM micrograph and backscatter of sterling silver cross-section (5-week

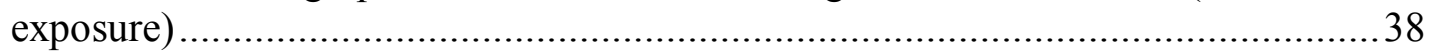

Figure 36: SEM micrograph and backscatter of sterling silver cross-section (7-week

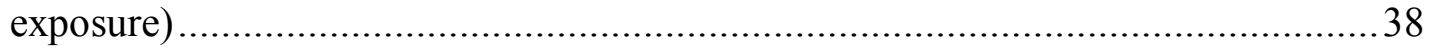


Figure 37: SEM micrograph and backscatter of sterling silver cross-section (10-week

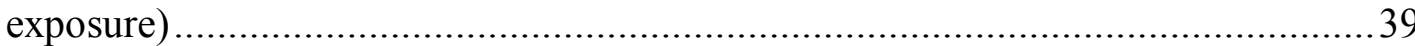

Figure 38: SEM micrograph and backscatter of sterling silver cross-section (13-week exposure)

Figure 39: micrograph and backscatter of sterling silver cross-section (17-week exposure)

Figure 40: SEM micrographs of sterling silver surface before and after exposure to compressed air (3-day sample)...

Figure 41: SEM micrographs of sterling silver surface before and after exposure to

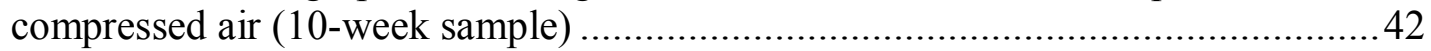

Figure 42: SEM micrograph of pure silver surface (no exposure).................................43

Figure 43: Pure silver (no exposure), SEM image and x-ray spectrum ............................44

Figure 44: Pure silver surfaces for high-temperature exposure .....................................45

Figure 45: Time-series analysis for early-stage sterling sample, low-magnification .......48

Figure 47: Time-series analysis for later sterling silver sample, low-magnification........52

Figure 48: Time-series analysis for later sterling silver sample, high-magnification........54

Figure 49: micrograph and backscatter of pressed $\mathrm{Ag} / \mathrm{CuO}$ sample surface....................56

Figure 50: SEM micrograph and $\mathrm{x}$-ray spectrum of pressed $\mathrm{Ag} / \mathrm{CuO}$ sample .................56

Figure 51: Time-series, pressed $\mathrm{Ag} / \mathrm{CuO}$ sample, low-magnification .............................58

Figure 52: Time-series, pressed $\mathrm{Ag} / \mathrm{CuO}$ sample, low-magnification .............................60

Figure 53: Ag/LSM sample surface with x-ray spectrums ...........................................61

Figure 54: Time-series, pressed Ag/LSM sample, low-magnification .............................62

Figure 55: Time-series, pressed Ag/LSM sample, high-magnification ...........................64

Figure 56: Comparison of faceting of sterling silver sample and pure silver sample (2-

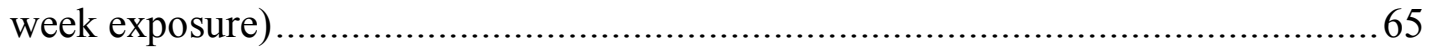

Figure 57: Comparison of faceting of sterling silver sample and pure silver sample (7-

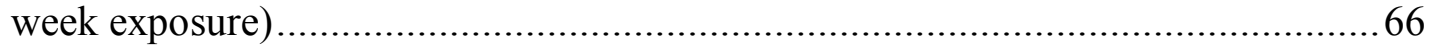




\subsection{BACKGROUND}

\section{CHAPTER 1: Introduction}

The solid-oxide fuel cell uses a dense oxygen-ion conducting compound as the electrolyte, making it simpler in concept than many other types of fuel cell systems, such as the phosphoric acid or molten carbonate fuel cells ${ }^{1}$.

Typical operating temperatures for the SOFC are between $800 \mathrm{C}$ and $1000 \mathrm{C}$. There are some advantages to operating at such a high temperature. The waste heat generated by the SOFC can be used for room heating or the generation of electricity by steam turbine regeneration ${ }^{21}$.

Several designs have been developed regarding the SOFC, including the planar, tubular and monolithic designs. The planar design is of interest for this research. For this type of cell geometry, the components of the cell are fabricated as thin, flat plates. The dense electrolyte is typically fabricated by tape-casting, powder sintering, or chemical vapor deposition (CVD). The porous electrodes are applied by slurry methods, screen printing, or plasma spraying. The advantage of this design is high power density, however, this design has problems with regards to fabricating acceptable seals at the edges that can facilitate the high operating temperatures of the $\mathrm{SOFC}^{2}$.

The major components of the SOFC are the cathode, anode, electrolyte, and interconnect.

The anode is typically a cermet made of yttria-stabilized zirconia (YSZ) and nickel. Nickel is chosen for its high electronic conductivity as well as stability under chemically reducing conditions ${ }^{1}$. Also, the YSZ skeleton formed around the nickel particles prevents sintering of the nickel as well as decreases the thermal expansion of the cermet, more in line with other cell components ${ }^{2}$. 
The cathode is typically made up of electronically conducting oxides or mixed electronically conducting and ion-conducting ceramics, usually strontium-doped lanthanum manganite (LSM) ${ }^{1}$. The cathode must meet several requirements such as high electronic conductivity, thermal expansion in line with other cell components, compatibility and minimum reactivity with the electrolyte and the interconnection with which the air electrode comes into contact, and sufficient porosity to facilitate transport of molecular oxygen from the gas phase to the electrode/electrolyte interface components $^{2}$.

The electrolyte of the SOFC is made of yttria-stabilized zirconia (YSZ), typically the zirconia is doped with 8 to $10 \mathrm{~mol} \%$ yttria. At temperature above $800 \mathrm{C}$ zirconia becomes a conductor of oxygen ions ${ }^{1}$.

The interconnect is used to connect adjacent cells in a fuel cell stack. Typically metals have been used to fabricate the interconnect, however these tend to be very expensive for high-temperature fuel cell applications. Metal interconnects also tend to form non-conductive-oxide coatings, which can reduce the stack efficiency by increasing the ohmic resistance of the system ${ }^{1}$.

\subsection{PRINCIPLE OF OPERATION OF SOLID-OXIDE FUEL CELL}

The electrochemical reactions occurring in SOFC's utilizing $\mathrm{H}_{2}$ and $\mathrm{O}_{2}$ are based on the following equations ${ }^{1}$ : 


$$
\begin{aligned}
& \mathrm{H}_{2}+\mathrm{O}_{2} \rightarrow \mathrm{H}_{2} \mathrm{O}+2 e^{-} \text {, at the anode } \\
& 1 / 2 \mathrm{O}_{2}+2 e^{-} \rightarrow \mathrm{O}^{2-} \text {, at the cathode } \\
& \mathrm{H}_{2}+1 / 2 \mathrm{O}_{2} \rightarrow \mathrm{H}_{2} \mathrm{O} \text {, overall cell reaction }
\end{aligned}
$$

The oxygen supplied at the anode reacts with incoming electrons from the external circuit to form oxygen ions, which migrate to the anode through the oxygen-ionconducting electrolyte. At the anode, oxygen ions combine with $\mathrm{H}_{2}$ to form $\mathrm{H}_{2} \mathrm{O}$, liberating electrons ${ }^{2}$. This process is illustrated in the schematic drawing below.

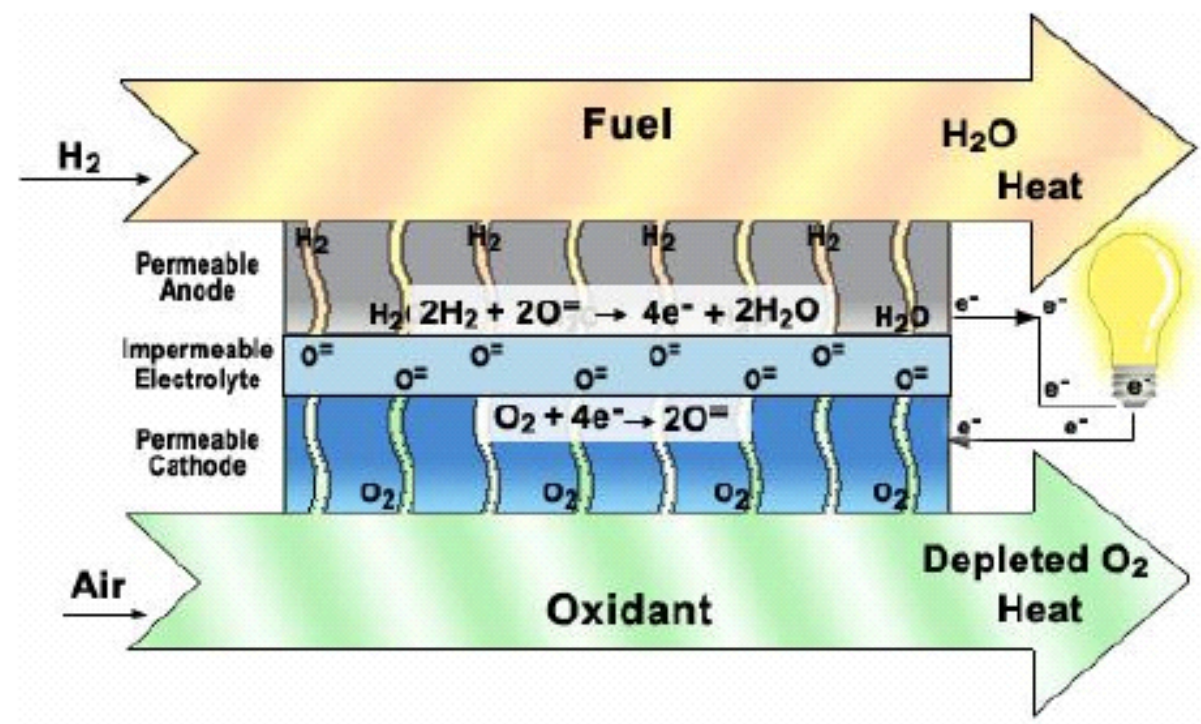

Figure 1: Illustration of chemical reaction in a solid-oxide fuel cell

\subsection{OPERATING VOLTAGE}

The operating voltage of the SOFC is equal to the open-circuit voltage minus the various losses associated with fuel cell operation. The four major types of losses are ohmic losses, activation losses, fuel crossover losses, and mass transport or concentration $\operatorname{losses}^{2}$ (Fuel Cell Systems Explained, 2 ${ }^{\text {nd }}$ Edition, James Larminie and Andrew Dicks). 
Ohmic losses are those losses associated with the resistance to the flow of electrons through the components of the cell (interconnect, electrolyte, electrodes, etc.) and is the main concern for this research. Voltage lost to driving the chemical reactions at the surface of the electrodes are termed activation losses. Fuel crossover losses are associated with fuel that is wasted passing through the electrolyte. Mass transport and concentration losses are due to the change in concentration of reactants at the surface of the electrodes as the fuel is used ${ }^{1}$. The following equation illustrates how one would calculate the cell operating voltage:

$$
V_{o p}=V_{o c v}-\Delta V_{o h m}-\Delta V_{a c t}-\Delta V_{\text {trans }}
$$

Equation 1: Calculation of cell operating voltage

$$
\Delta V_{o h m}=I R
$$

\section{Equation 2: Calculation of Ohmic Loss}

In the above equation $R$ is the resistance of the cell components. This resistance can increase or decrease based on the materials used in the fabrication of the cell stack, as well as the geometry of the cell components (i.e. thickness). An increase in resistance of the materials will lead to a decrease in the operating voltage of the stack, reducing cell performance. Therefore it is very important that low-resistance materials be used to fabricate the cell and its components.

In current planar SOFC designs the ohmic resistance is kept low by fabricating cell components as thin as possible. 


\subsection{RESEARCH OBJECTIVE}

The objective of this research project is to determine a suitable material to be used as a contact layer between the cathode and interconnect in the solid-oxide fuel cell (SOFC). The area of concern is shown in the schematic below illustrating a two-cell fuel cell stack.

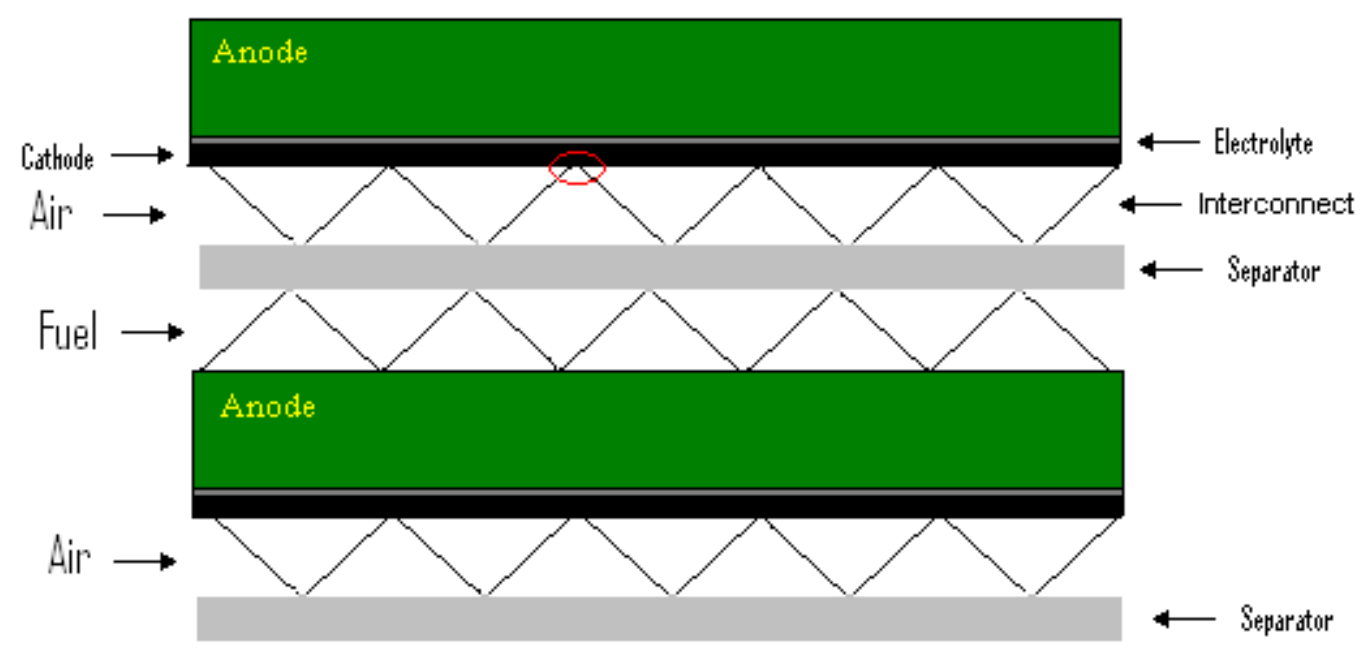

Figure 2: Schematic of SOFC stack

The major area of interest of this research is the contact areas in the cathode channel and their ability to maintain the electrical connections between cells during operation. Also, oxidation could be in issue due to the high temperature air stream in the cathode channel. Oxidation could prove detrimental to the cell if non-conducting oxide scales form, cutting off the electrical path between the cells.

A closer look at the contacting area is shown in the following figure. 


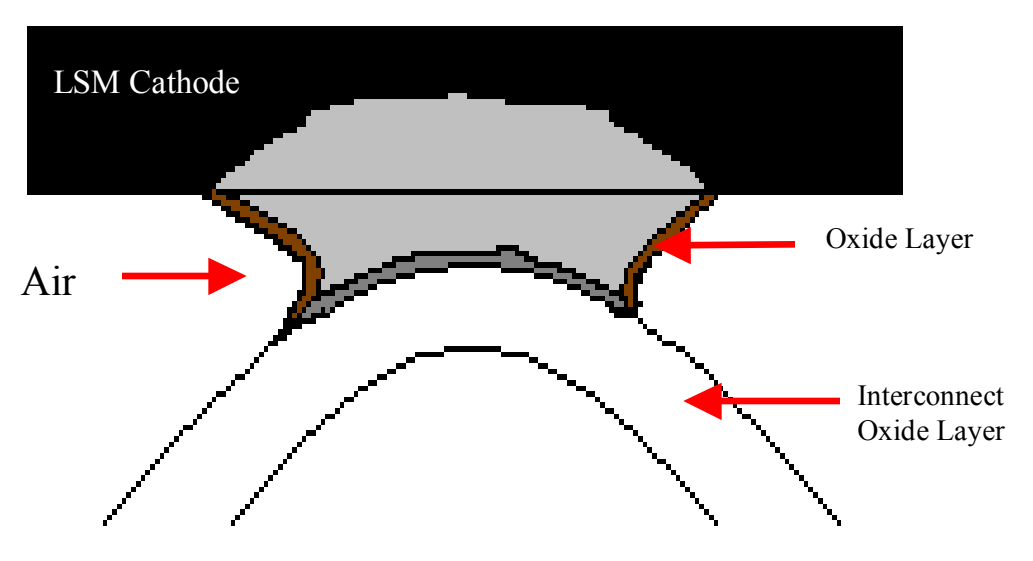

Figure 3: Schematic of contact area between interconnect and LSM cathode

The cathode chamber is an oxidizing environment, making it difficult to use many metals as they tend form a non-conductive scale which could reduce the efficiency of the stack by cutting off the electron path through the cells. Another major concern with regards to the maintenance of the conduction path is the evaporation of the conducting material. Ideally, the chosen material will be able to grow a protective skin that will protect the conducting metal, while not cutting off the electron path and reducing the efficiency of the fuel cell stack.

Research into the area of contacting materials has already been undertaken at the Pacific Northwest National Laboratory (PNNL) ${ }^{9}$. Various materials were examined with respect to area specific resistance (ASR), including platinum, LSM, LSC (strontiumdoped lanthanum cobalite), LSF (strontium-doped lanthanum ferrite) as well as operation with no contacting material at all. Testing was conducted at $800 \mathrm{C}$ with the materials applied between Crofer22 APU coupons and LSF cathodes. It was found the platinum performed best, but is cost prohibitive, while operating with no contacting material gave 
the worst performance. Of the other materials LSM showed the slowest rate of increase in ASR. Silver was not examined in this study.

Silver has more recently become a viable option due to the lowering of SOFC operating temperatures to around $800 \mathrm{C}$ from $1000 \mathrm{C}$. Silver also possesses the highest room temperature conductivity of any metal ${ }^{12}$. Good ductility, and low cost relative to other SOFC materials make silver an attractive choice for use in SOFC systems. Also, silver does not form a non-conducting scale when exposed to an oxidizing environment such as that of the cathode chamber in the SOFC. The various properties of silver are shown in the table below.

\begin{tabular}{|l|l|}
\hline \multicolumn{2}{|c|}{ Properties of Silver } \\
\hline Atomic Mass & $107.86 \mathrm{~g} / \mathrm{mol}$ \\
\hline Conductivity & \\
\hline Thermal & $4.29 \mathrm{~W} / \mathrm{cmK}$ \\
\hline Electrical & $0.6310 \mathrm{e} 6 / \mathrm{cm} \Omega$ \\
\hline Density & $10.48 \mathrm{~g} / \mathrm{cc}$ \\
\hline Moduli & \\
\hline Young's & $83 \mathrm{GPa}$ \\
\hline Bulk & $100 \mathrm{Gpa}$ \\
\hline Rigidity & $30 \mathrm{GPa}$ \\
\hline Melting Point & $961 \mathrm{C}$ \\
\hline Boiling Point & $2163 \mathrm{C}$ \\
\hline Elec. Resistivity & $1.467 \mathrm{e}-8 \Omega-\mathrm{m}$ \\
\hline Hardness & \\
\hline Vickers & $251 \mathrm{MN} / \mathrm{m}$ \\
\hline Brinell & $24.5 \mathrm{MN} / \mathrm{m}$ \\
\hline Mohs & 2.5 \\
\hline
\end{tabular}

Table 1: Properties of silver 
Research into silver evaporation has been conducted in Julich, Germany by Muelenberg et $\mathrm{al}^{11}$. Silver meshes were exposed to air flow at $800 \mathrm{C}$ as well as exposed in inert gas for a duration of 500 hours. The results show that silver evaporation rate is much greater in air than inert gas. It was also found that silver can maintain a low ASR over long-term exposure to cathode conditions as no poorly conducting corrosion materials form on silver ${ }^{11}$. This examination did not attempt to use any types of oxides to inhibit the rate of silver evaporation.

Pure silver is often too soft for most uses. Sterling silver was the material of main consideration for this study due to its favorable composition and low cost. Sterling silver is silver-copper alloy. The composition of sterling silver is $7.5 \mathrm{wt} \%$ copper-silver. The addition of copper causes the silver to become less pliable and stronger in its structure, improving strength and durability, allowing the fabrication of items that will retain their shape after use. A common use of sterling silver is the manufacture of silverware and jewelry.

Silver-Copper alloys form copper-oxide both in the external scales and within the alloy upon oxidation ${ }^{4}$. Copper only forms two thermodynamically stable oxides, $\mathrm{CuO}$, copper-oxide and $\mathrm{Cu}_{2} \mathrm{O}$, cuprous oxide ${ }^{7}$. When exposed to oxygen, copper will naturally oxidize, forming $\mathrm{Cu}_{2} \mathrm{O}$, cuprous oxide, which is red in color, however, this takes extensive periods of time. This process can be accelerated by heating copper at high temperatures or at high oxygen pressure. With further heating, $\mathrm{Cu}_{2} \mathrm{O}$ will form $\mathrm{CuO}$, a black solid which melts at about $1345 C^{8}$. Upon exposure, the copper becomes copperoxide $(\mathrm{CuO})$, which is meant to protect the silver from evaporation, increasing the life 
expectancy of the cermet and allowing the SOFC to operate longer. The target operation time is 40,000 hours.

Copper-oxide may have some issues regarding electron conductivity when used as a cathode material, however it should have appropriate oxygen-ion conductivity ${ }^{6}$. 


\section{CHAPTER 2: Experimental}

\subsection{EXPERIMENTAL}

\subsubsection{Sample Preparation}

The samples for this experiment were small sterling silver plates. The average size of the sterling samples was $11 \mathrm{~mm}$ X $6 \mathrm{~mm} X 0.7 \mathrm{~mm}$. The samples were first cut from a bar of sterling silver using an Isocut slow speed saw, followed by cold pressing in a bench vise using a $13 \mathrm{~mm}$ die set. Afterward, the samples were cut again to the shape of a rectangle, and sample dimensions were measured using calipers. The samples were then cleaned using acetone and their masses recorded using an electronic scale.

Pure silver samples were also fabricated for comparison with their sterling silver counterparts. Two types of pure silver samples were used. One sample-type very thin ( $\sim 50 \mu \mathrm{m}$ in thickness) and one thick $(\sim 700 \mu \mathrm{m}$, similar to the sterling samples). These pure silver samples were cut, measured, cleaned in acetone and weighed in the same manner as the sterling silver samples.

The experimental procedure for this research involved creating an environment similar to what the material would be exposed to in a real functioning fuel cell. This was accomplished using a simple tube furnace setup, illustrated in the following schematic: 


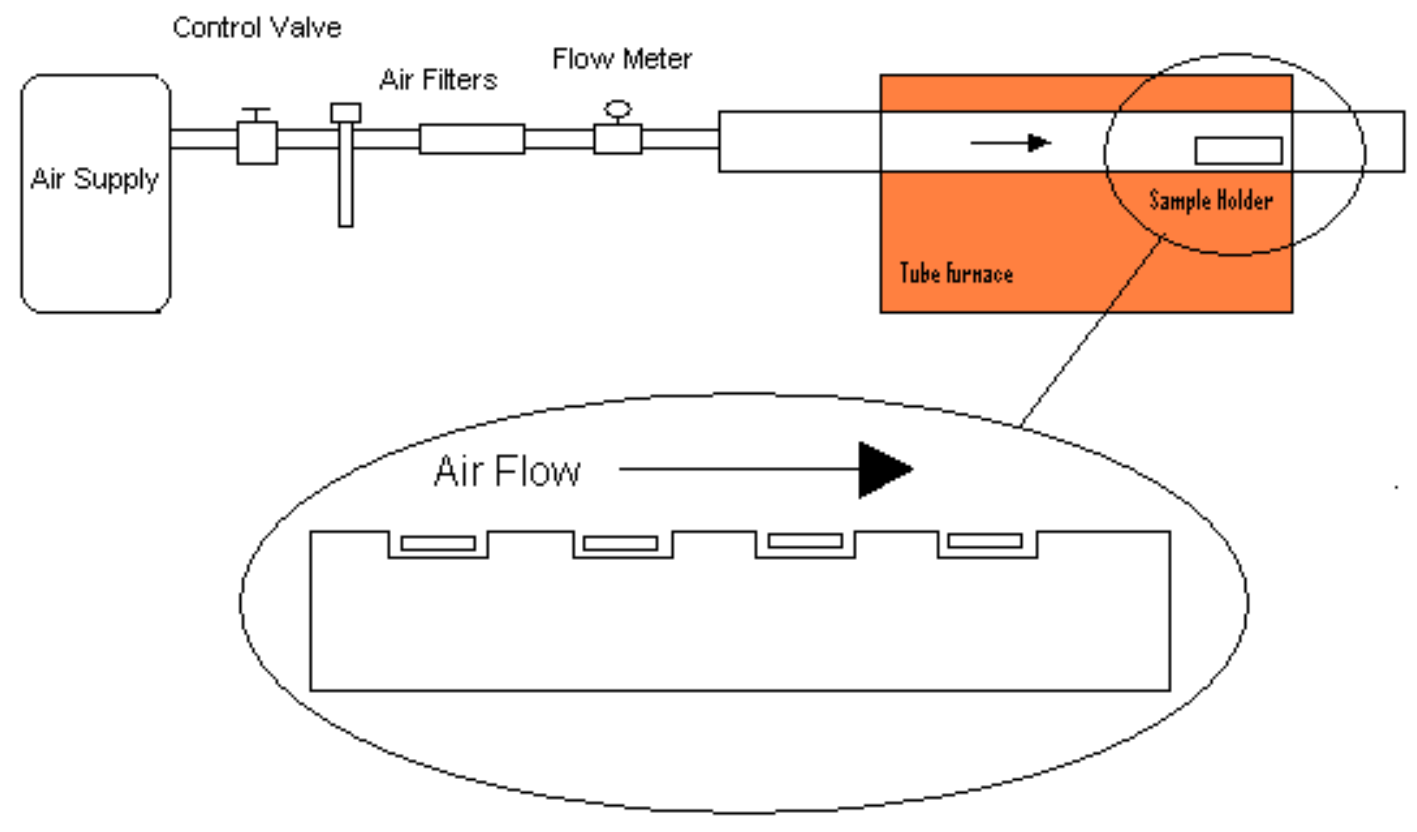

Figure 4: Experimental setup for exposure of samples

This setup allows sample to be placed inside the furnace which is maintained at $800 \mathrm{C}$, while air is flowed through the tube. Ceramic inserts were cut and placed between the sample to reduce the possibility of cross-contamination of the samples. A schematic drawing of the sample holder is shown in the following figure:

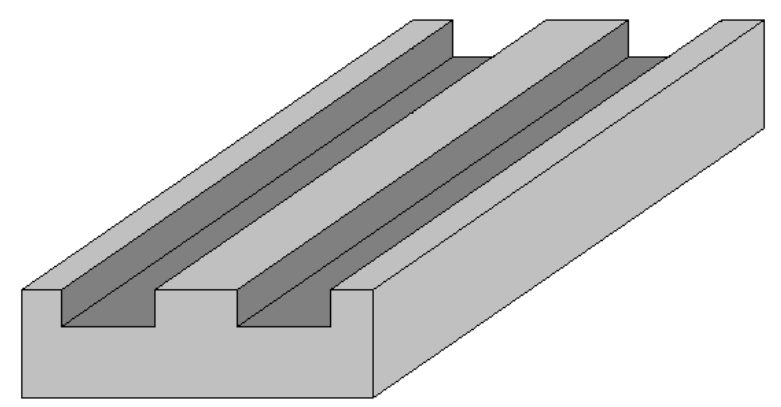

Figure 5: Sample holder for exposure experiment 
A simple valve was used to control the air supplied to the system, which is measured using a Sierra Smart-Trak Series 100 flow meter. The flow rate of the air through the heated tube furnace was calculated to be $31 / \mathrm{min}$.

All samples were measured each week to examine any changes in mass that occurred due to exposure in the furnace. Each week a sample was also set aside for SEM evaluation of any changes in microstructure. The mass changes were then used to estimate reduction in thickness due to silver loss.

Sterling samples were also pre-marked for SEM analysis before exposure so as to allow examination of the same surface sight repeatedly to offer a more detailed understanding of the changes in the microstructure at the surface of the sample.

\subsection{CALCULATIONS}

In order to determine sample performance during cell operation some basic calculations were carried out based on the data obtained from the experiment. The main property of concern with was the thickness reduction of the sample during exposure. Thickness reduction eliminates variation in sample changes that may be due to a difference in sample dimensions, which is important in this case as all of the samples were fabricated in-house using raw materials. Thickness reduction was calculated using the following formula:

$$
\text { Thickness Re duction }=\left(\frac{\text { post evap mass }- \text { pre evap mass }}{(\text { Exposed Surface Area })(\text { Density of Silver })}\right)
$$

\section{Equation 3: Calculation of thickness reduction}


This formula assumes that there is negligible change in the length or the width of the sample and that the loss in mass of the sample is due entirely to a loss in silver, with negligible loss of the copper-oxide.

Each week every sample was weighed multiple times and the average was taken to be the mass, this process also provided the standard deviations used in the error calculation. 


\subsection{RESULTS}

The sterling samples all followed a similar trend; after an initial gain in mass due to the oxidation of the copper, the samples began to loss mass at a relatively steady rate over the course of several weeks. Based on this loss of mass, the thickness reduction of the silver at the surface of the sample was calculated using the equation above. The results over the duration of the testing with error bars are shown in the following bar chart:

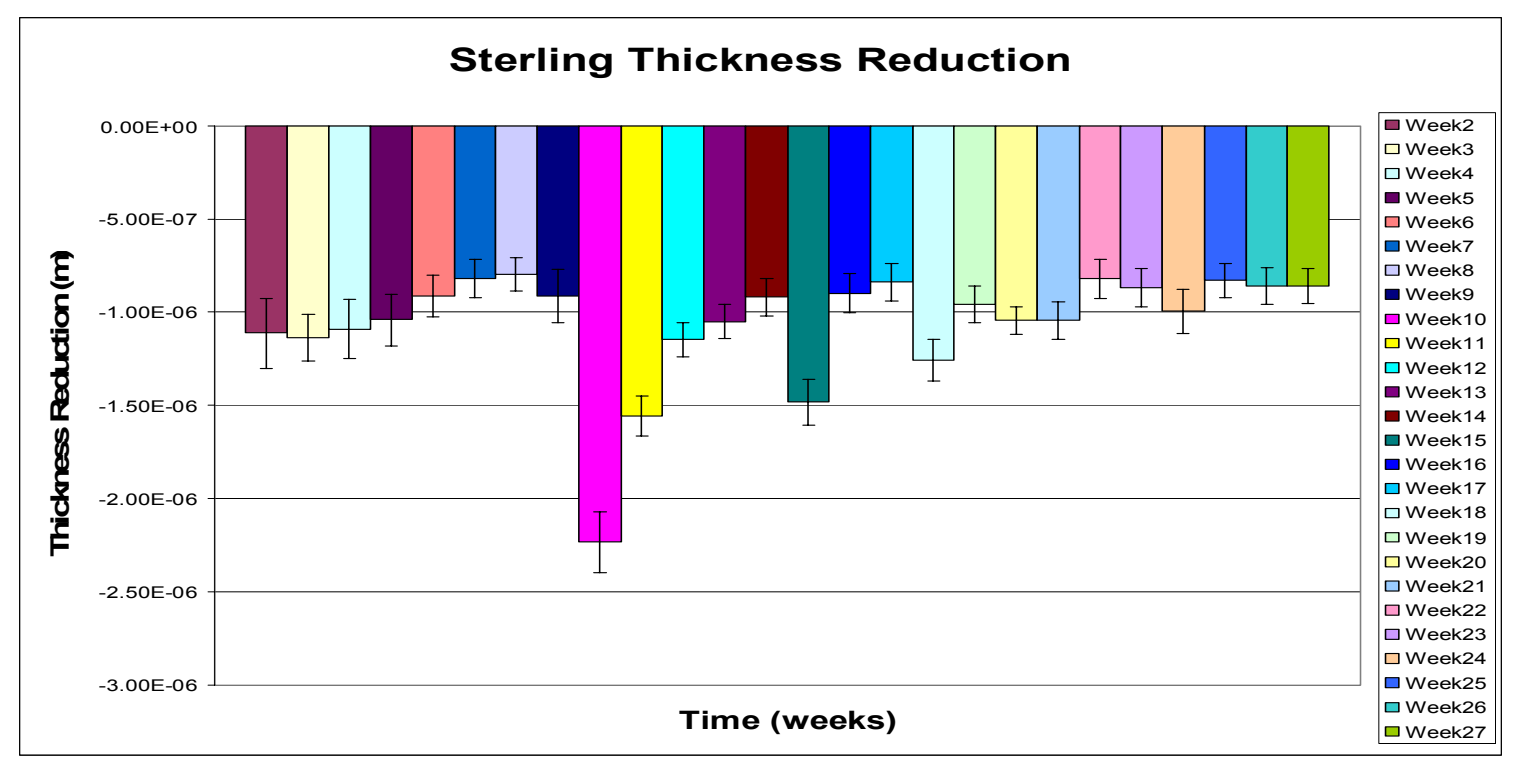

Figure 6: Thickness reduction of sterling silver

The accumulation of the thickness reduction was also plotted and is shown in the figure below for the sterling silver samples. 


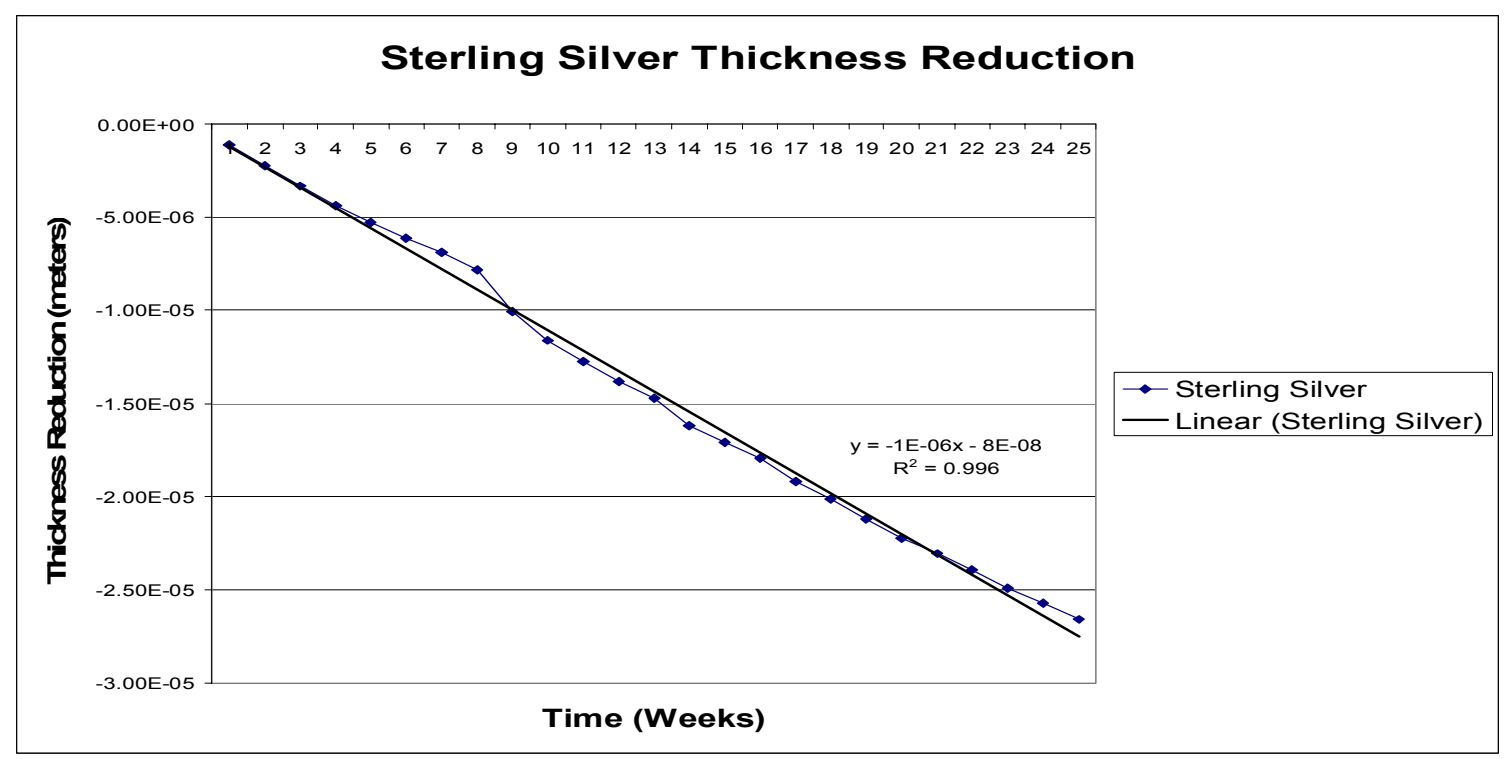

Figure 7: Accumulating thickness reduction of sterling silver

From the plot it appears that the reduction in thickness is roughly linear over the duration of the sample exposure. A linear trend of this line was used to approximate a rate of thickness reduction in the sample to allow prediction of the sample deterioration after the 40,000 hour target time. It appears that the sample loses about 1 micron in thickness each week, meaning that over the course of 240 weeks $(40,000 \mathrm{hrs})$, the sample would lose about $0.24 \mathrm{~mm}$ in thickness.

Pure silver samples were also placed in the furnace for evaluation of their thickness reduction during exposure. Two types of pure silver samples were examined in the high-temperature exposure experiment. A thin sample, roughly 50 microns thick, and a thicker sample, roughly 700 microns, were used. The thicker sample was similar in thickness to the sterling silver samples. The pure samples of different thickness were used to determine if there was a relationship between sample thickness and evaporation 
rate. The table below illustrates the variation in thickness reduction of the thin pure silver samples over the duration of the experiment.

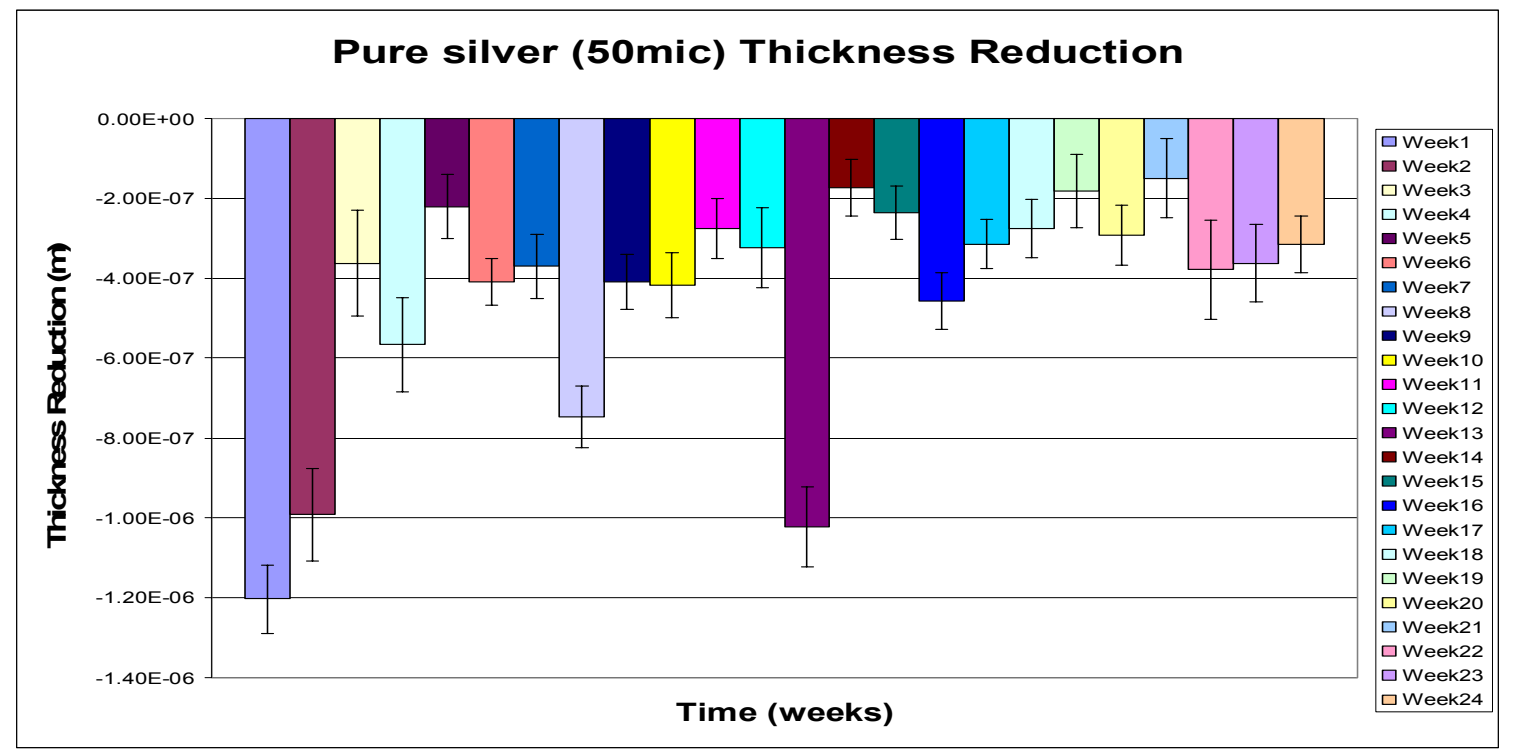

Figure 8: Thin pure silver thickness reduction

The data shows that the general trend for the pure silver samples is a reduction in the thickness reduction as the experiment proceeds. Also, though there is more variation in the loss from week to week than with the sterling silver samples, the values are still within the error bars for the most part, indicating that the loss rate is still relatively consistent. 


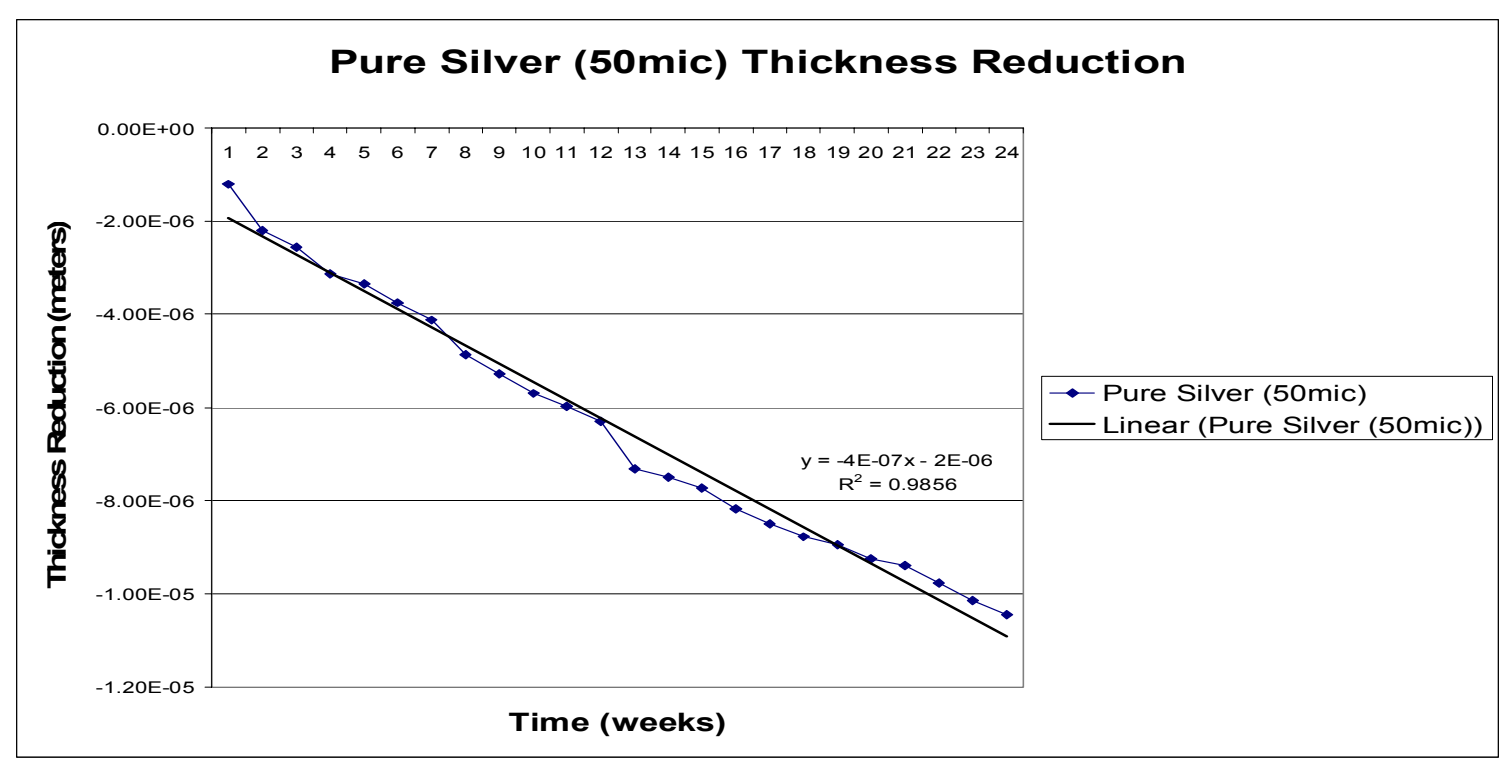

Figure 9: Accumulating thickness reduction of pure silver

Similar to the plot for sterling silver, it appears that the reduction in thickness of the pure silver specimen is also occurring at a relatively constant rate, however, the total reduction in thickness of the pure silver seems to be lower than that of the sterling silver. This result is surprising as it was initially expected that the copper-oxide in the sterling samples would act to slow their evaporation rate. The rate of loss for the thin pure silver sample is roughly 0.4 microns per week, less than half of that of the sterling silver samples.

The thicker pure silver sample exhibited a greater thickness reduction than its thinner counterpart, however, over time it appears that the trend is consistent with the thinner silver sample. The plot below illustrates the variation in thickness reduction by week of the thicker pure silver sample. 


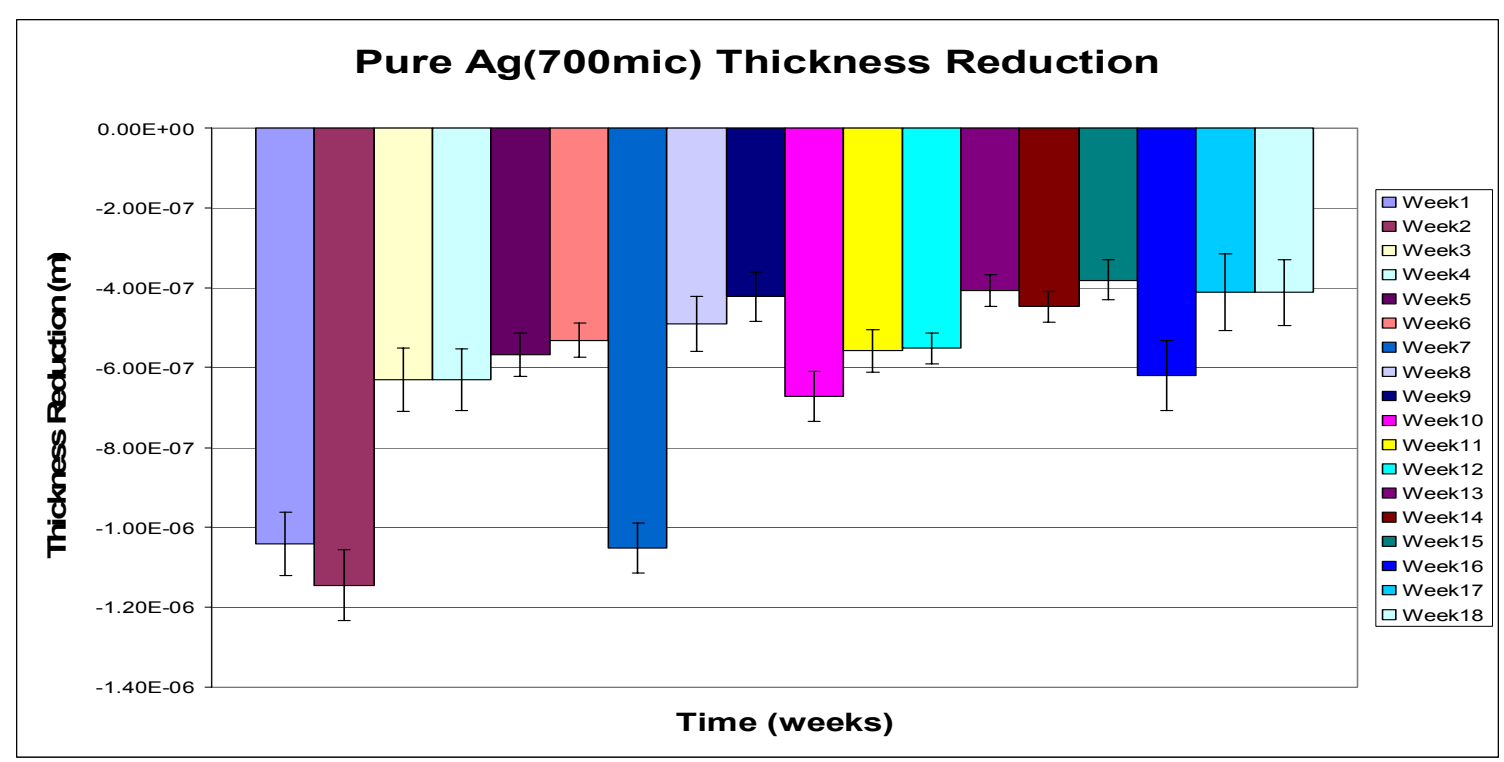

Figure 10: Thickness reduction of thick pure silver sample

The thickness reduction for the thicker pure silver sample shows a downward trend similar to the thin pure silver sample. The thickness reduction is decreasing over time as opposed to the constant thickness reduction illustrated by the sterling silver samples. The accumulating thickness reduction for the thicker sample was also plotted and is shown in the following figure. 


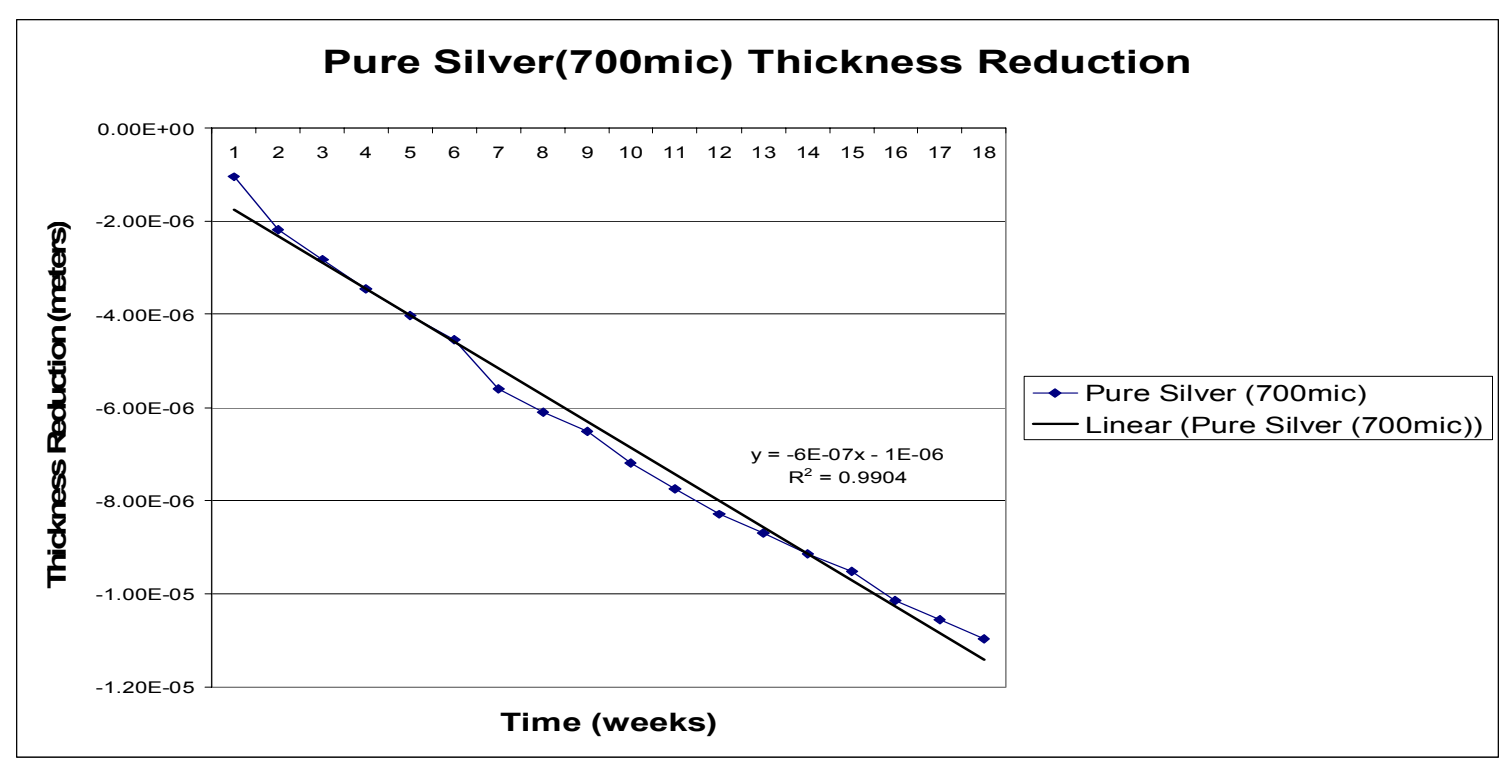

Figure 11: Accumulating thickness reduction of pure silver

The rate of loss per week of the 700 micron thickness pure silver sample is roughly 500 microns per week, similar to the rate of evaporation of the 50 micron thickness sample and a little more than half that of the sterling sample.

A comparison of all of the samples accumulating thickness reduction is shown in the following figure. 


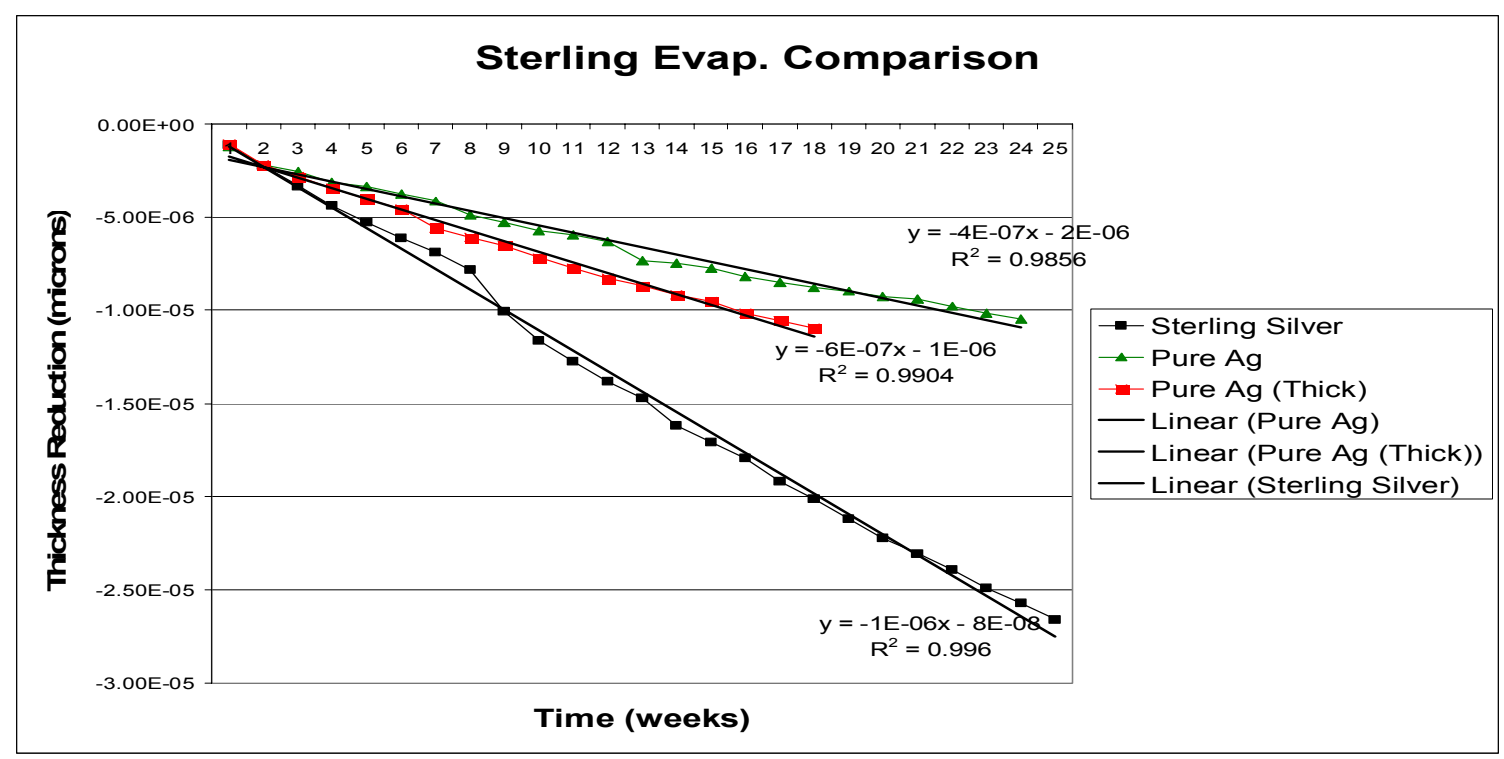

Figure 12: Comparison of thickness reduction for all types of samples

From this data it can be seen that the sterling silver samples lose mass more rapidly than the pure silver samples of differing thicknesses, which appear to exhibit similar behavior with regards to evaporation. This result is undesirable as it was hoped that the copper-oxide formed in the sterling silver samples would act to slow the rate of evaporation in the sample. Scanning electron microscopy of the sample surfaces was carried assist in determining possible causes of these results. 


\section{CHAPTER 4: Scanning Electron Microscopy}

\subsection{SEM ANANLYSIS}

In order to evaluate changes in microstructure of the samples during hightemperature exposure scanning-electron microscopy (SEM) was done daily for the first few days and then weekly to examine any changes that occur over the long-term. The images below illustrate some of the results. The first images illustrate the surface of a sterling sample before and after evaporation, also showing $\mathrm{x}$-ray spectrums of areas of the surface to verify the presence of silver and copper-oxide.

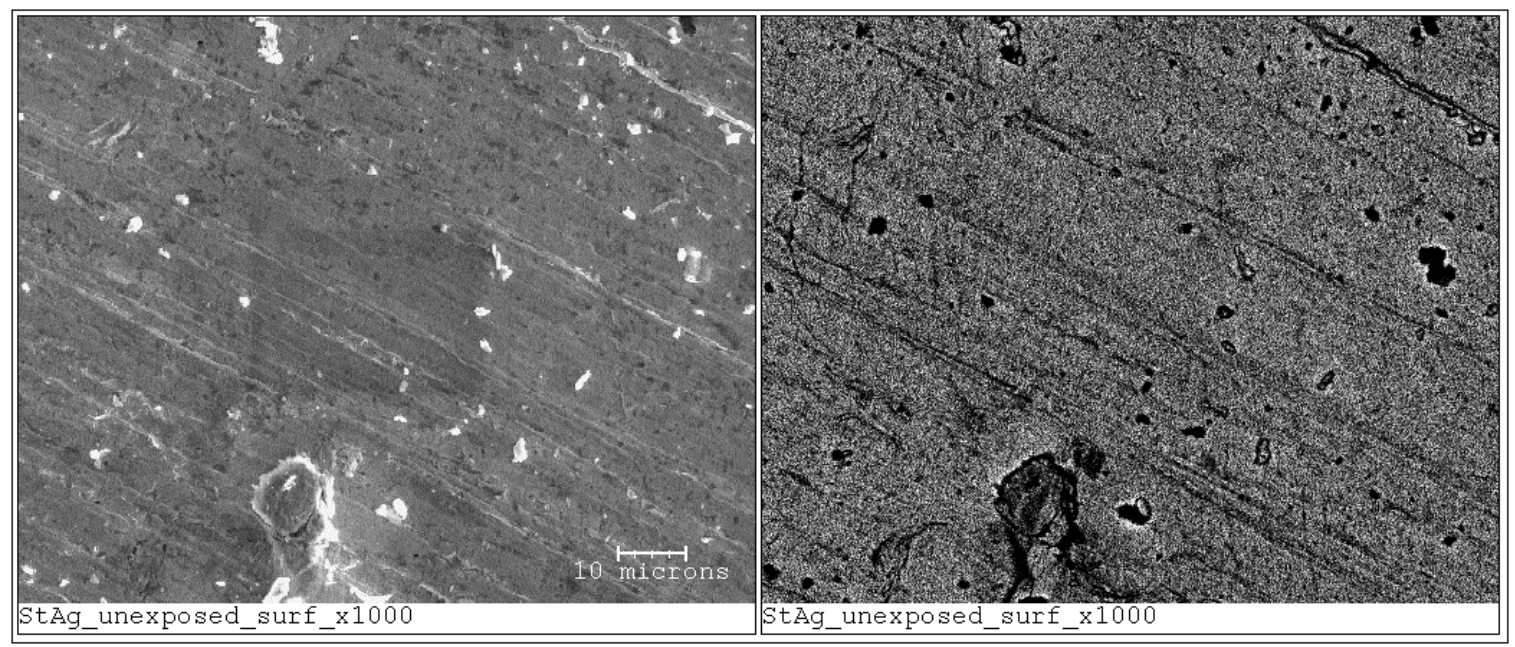

Figure 13: Unexposed sterling silver surface SEM micrograph and backscatter image

When using backscatter imaging, heavier elements appear lighter in color, while lighter elements appear darker in color. In the above image it can be seen that the majority of the surface of the sample is silver, which is lighter in color, while small dark particles indicate the presence of copper at the surface. 
The sample was also analyzed using EDS mapping. The elemental map of the surface is shown in the following figure.

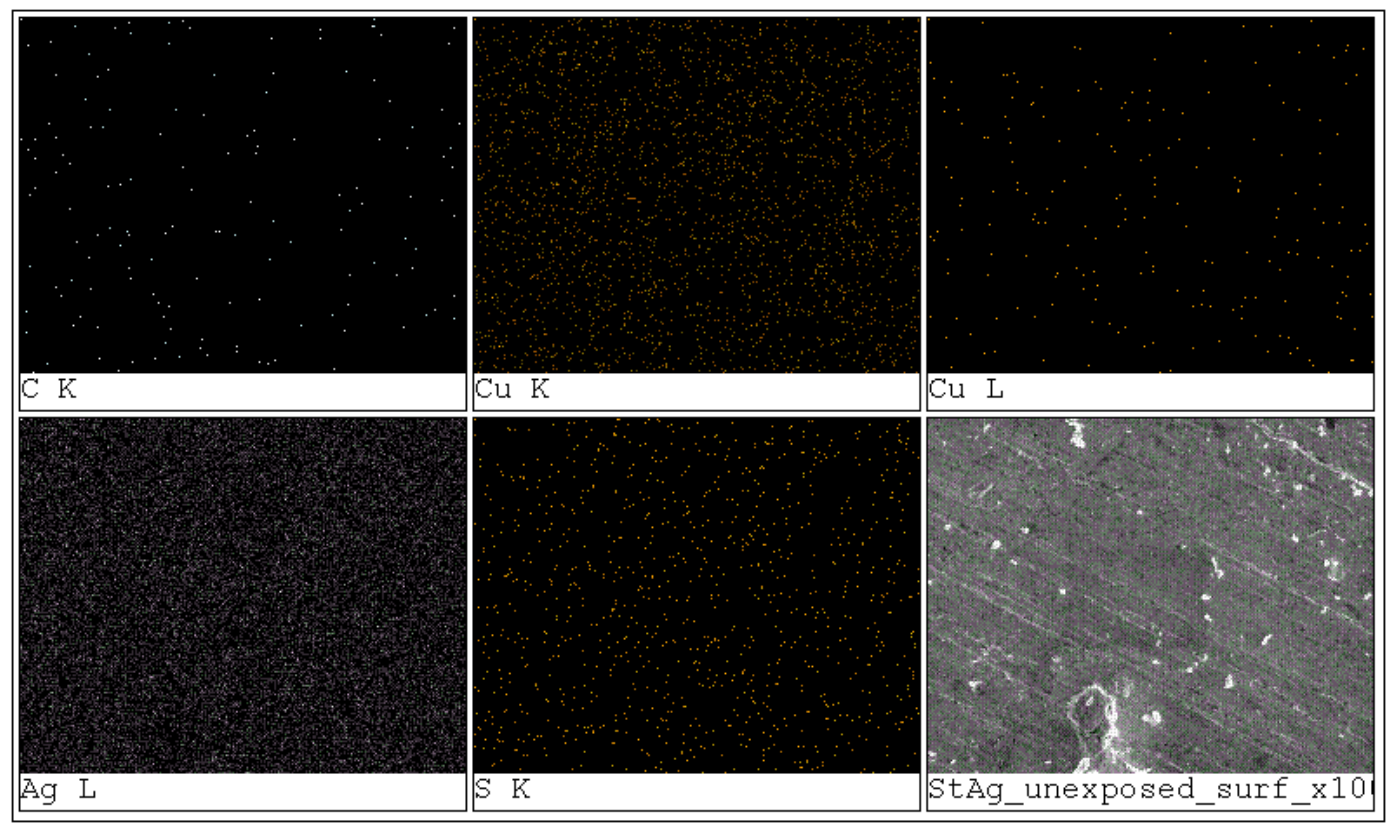

Figure 14: EDS of sterling silver surface

It can be seen in the EDS map that copper is abundant at the surface. This can also be seen in the backscatter as the dark spots in the image making it appear grainy. The images show that the copper is relatively evenly dispersed throughout the surface of the sterling sample, which is a desirable property of the sample.

Samples were oxidized in a box furnace with no air flow in order to determine the surface composition of a fully oxidized sample, which would theoretically provide the maximum copper-oxide coverage, and the most protection from evaporation to the silver. The sample was cycled a few hours at a time, its mass being measured between each cycle until the mass of the sample was no longer increasing. The mass of the sample no longer increased after roughly twelve hours in the box furnace. This point is assumed to 
be a state of full oxidation of the sample and the surface should illustrate maximum coverage of the silver by copper-oxide. The SEM micrograph with backscatter image is shown in the following figure.

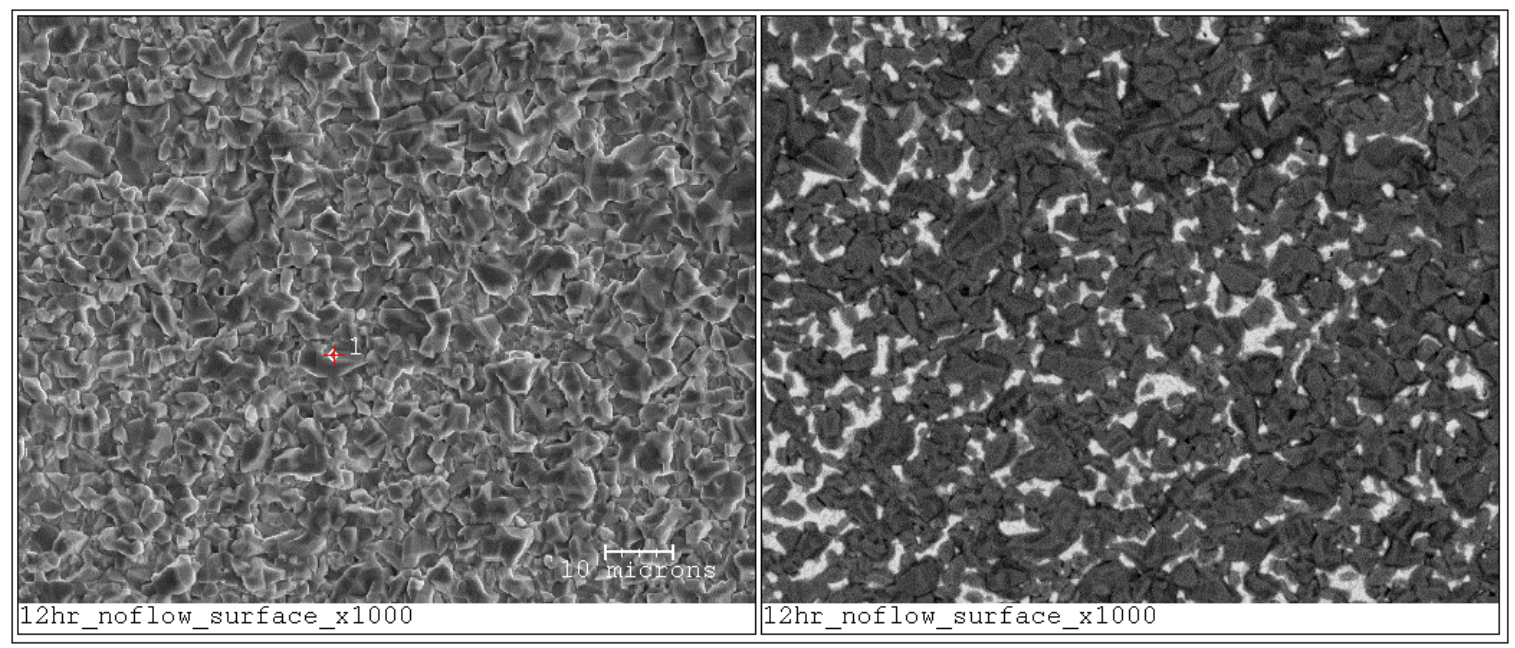

Figure 15: Sterling silver surface and backscatter after 12 hrs exposure with no air flow
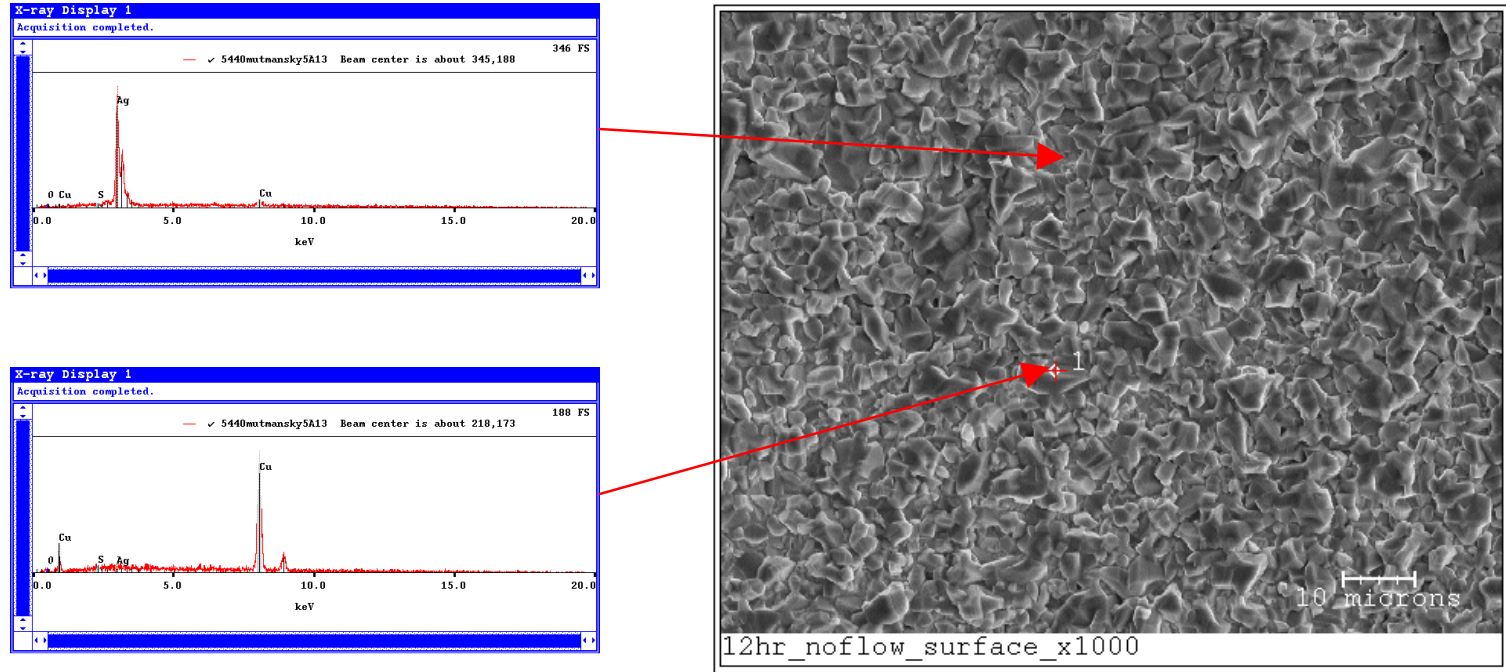

Figure 16: sterling silver surface and x-ray spectrum after 12 hour exposure 
After oxidation in the box furnace, the surface of the sample becomes relatively well covered with copper-oxide particles. It can be seen that the copper-oxide particles form as what appear to be "boulders" scattered throughout the surface. This orientation may be beneficial in the respect that it decreases the likelihood of spalation due to surface stresses caused by any thermal or mechanical stresses.

Samples exposed in the tube furnace were examined for changes in surface microstructure. The image below illustrates a typical surface image and x-ray spectrum used to identify silver and copper-oxide at the surface.

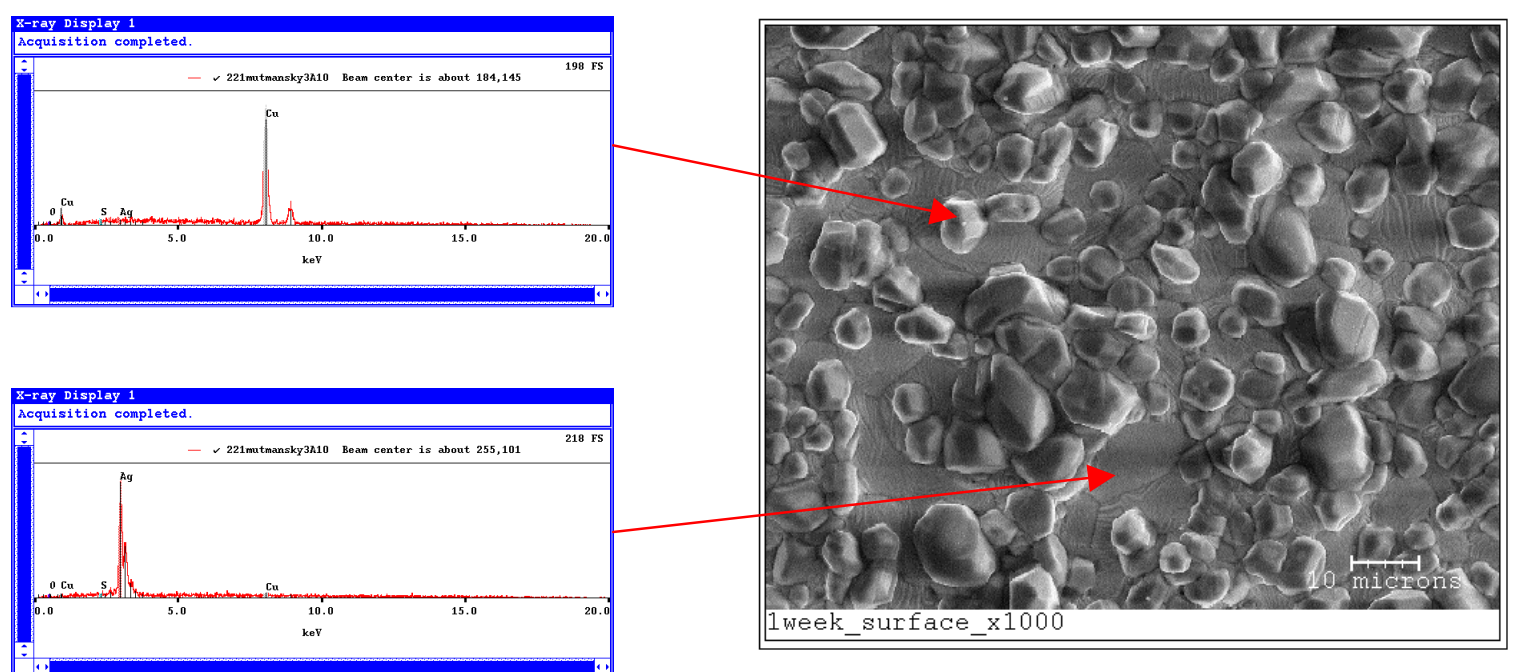

Figure 17: Sterling silver surface and x-ray spectrum after 1-week of exposure

From these images it is relatively easy to see the formation of copper-oxide at the surface of the sample, which is confirmed by the x-ray spectrum. The copper-oxide particles are much easier to identify after just one week of oxidation as compared to the unexposed sample, which only indicates a very small presence of copper at the sample surface. 
This sample was also analyzed using EDS for chemical composition. The elemental map of the surface is shown in the following figure.

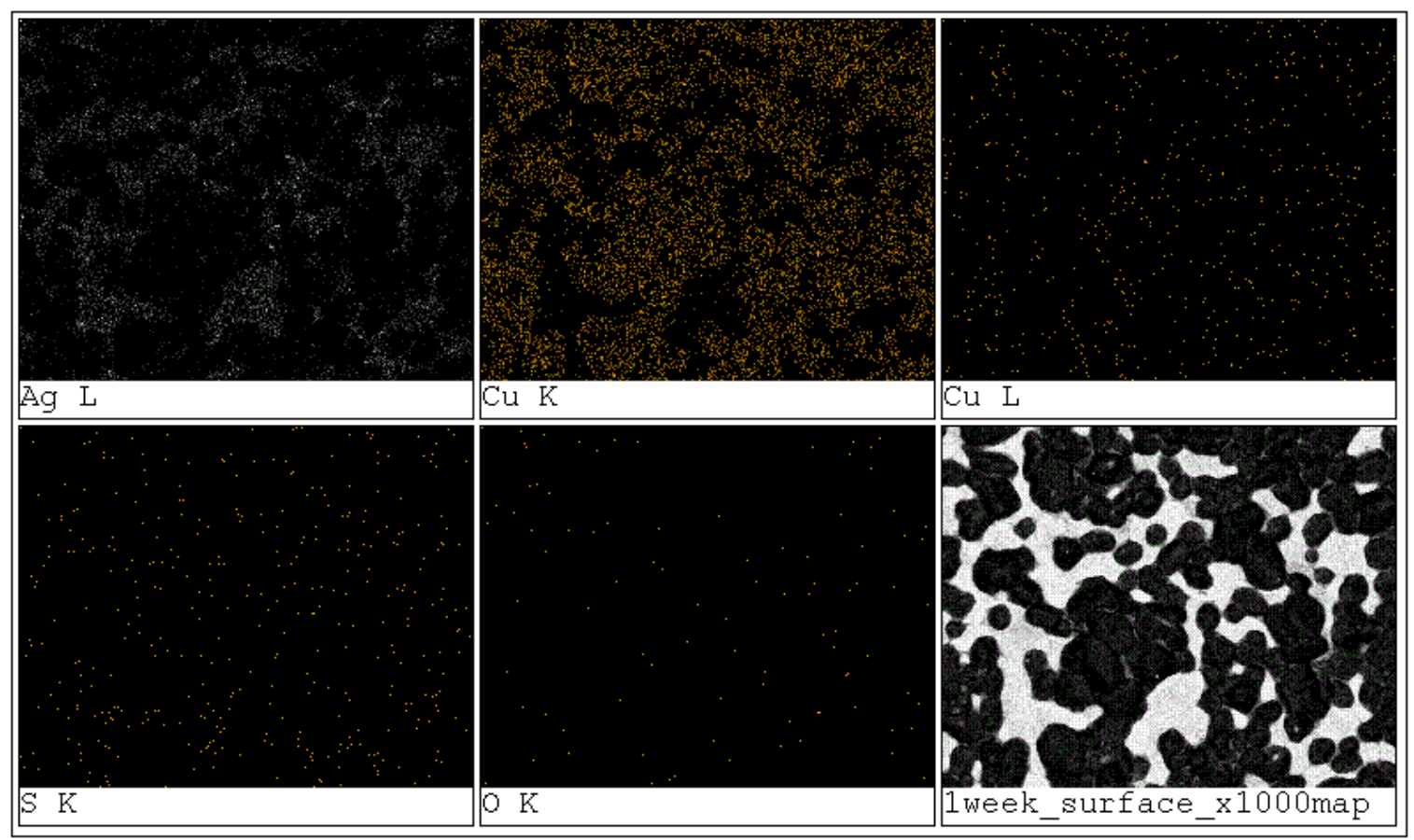

Figure 18: EDS of oxidized sterling silver sample

Backscatter imaging was used to identify the silver and copper at the surface of the sample after oxidation from this point onward. Backscatter imaging makes differentiation of silver and copper-oxide at the surface of the sample much more straightforward.

Several samples were subjected to high-temperature exposure. These samples were used to collect mass change data used to calculate thickness reductions. Samples were also periodically removed from the furnace to allow SEM analysis of any microstructure changes occurring at the surface. Scanning electron microscopy of the sterling sample surface is shown in the following figures. Each image shown in this set is a different sample, each having been exposed for differing durations. 


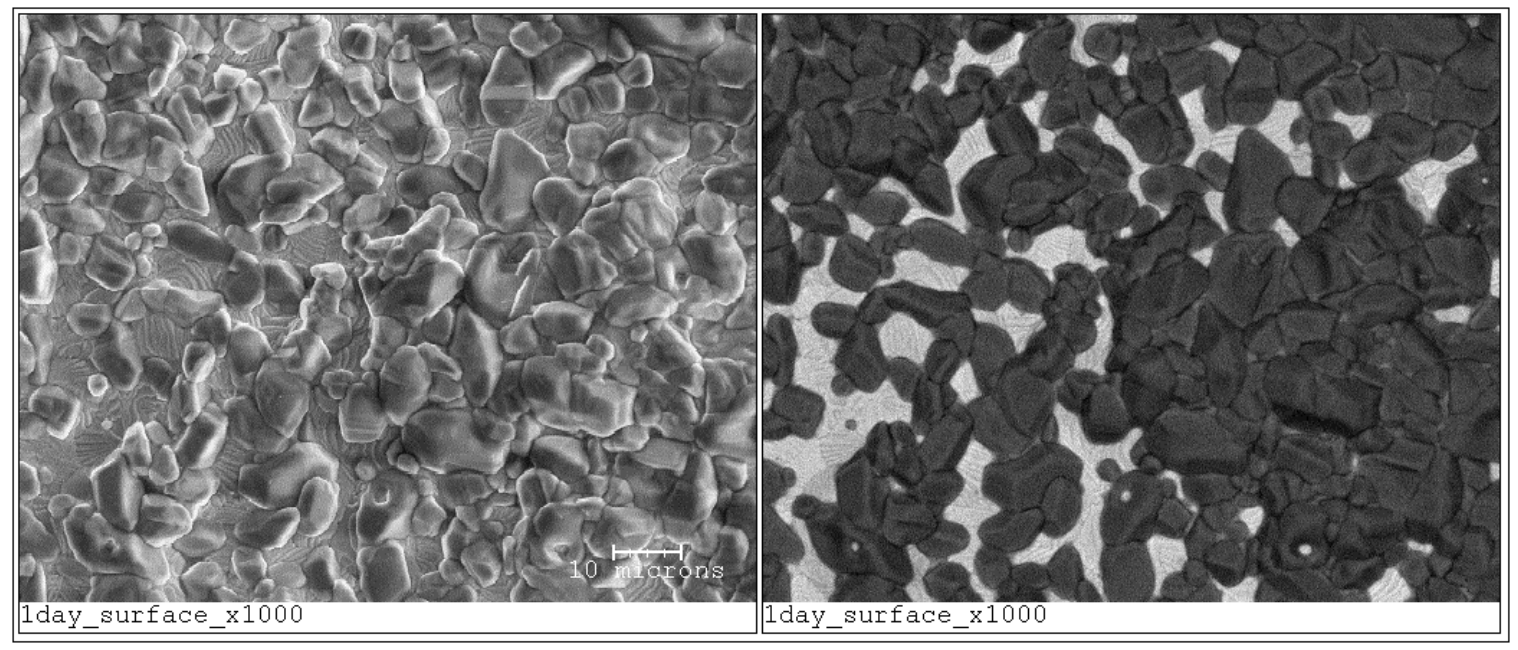

Figure 19: Sterling silver surface and x-ray spectrum after 1-day exposure

After one day of exposure copper-oxide particles can be seen to cover much of the surface of the sample and most of the particles appear to be in the range of $5-10 \mu \mathrm{m}$ in size. At this point it appears that there is relatively good coverage of the silver by the copper-oxide, calculation indicates that approximately $84 \%$ of the surface is covered; this copper-oxide could provide good protection against silver evaporation during cell operation. 


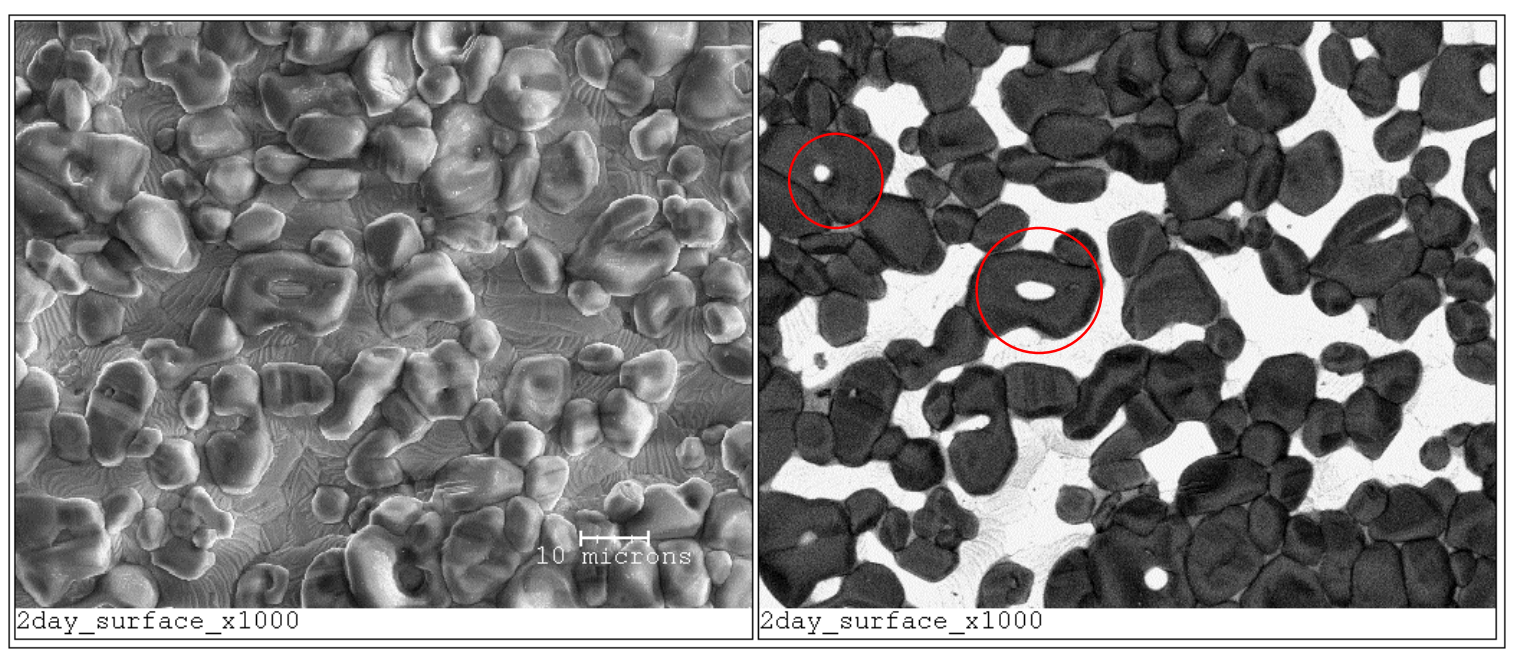

Figure 20: Sterling silver surface and x-ray spectrum after 2-day exposure

After two days of exposure, more silver is already visible at the surface of the sample and it also appears that some agglomeration of the copper-oxide is evident. The circled areas in the figure show what appear to be holes in large particles, however, these areas illustrate areas were several small copper-oxide particles are forming larger particles during high-temperature exposure. Calculations show that roughly $74 \%$ of the surface of the sample is covered by copper-oxide in this sample. 


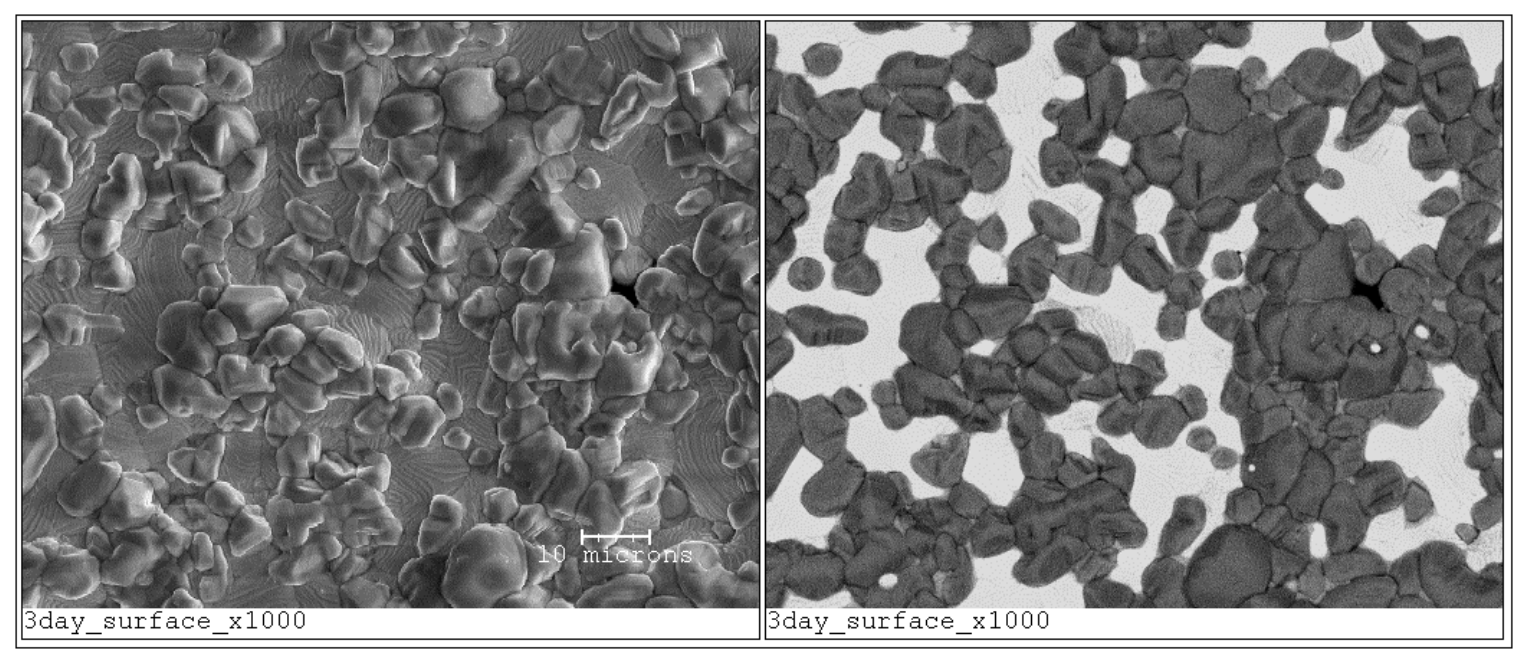

Figure 21: Sterling silver surface and x-ray spectrum after 3-day exposure

After three days of exposure, it can be seen that more silver is exposed at the surface of the sample, approximately $70 \%$ of the surface is covered by copper-oxide at this point based on calculation. Coarsening of the copper-oxide is also evident as particle sizes of $10 \mu \mathrm{m}$ and greater are visible in the micrograph, there is also an abundance of smaller particles still visible in the image.

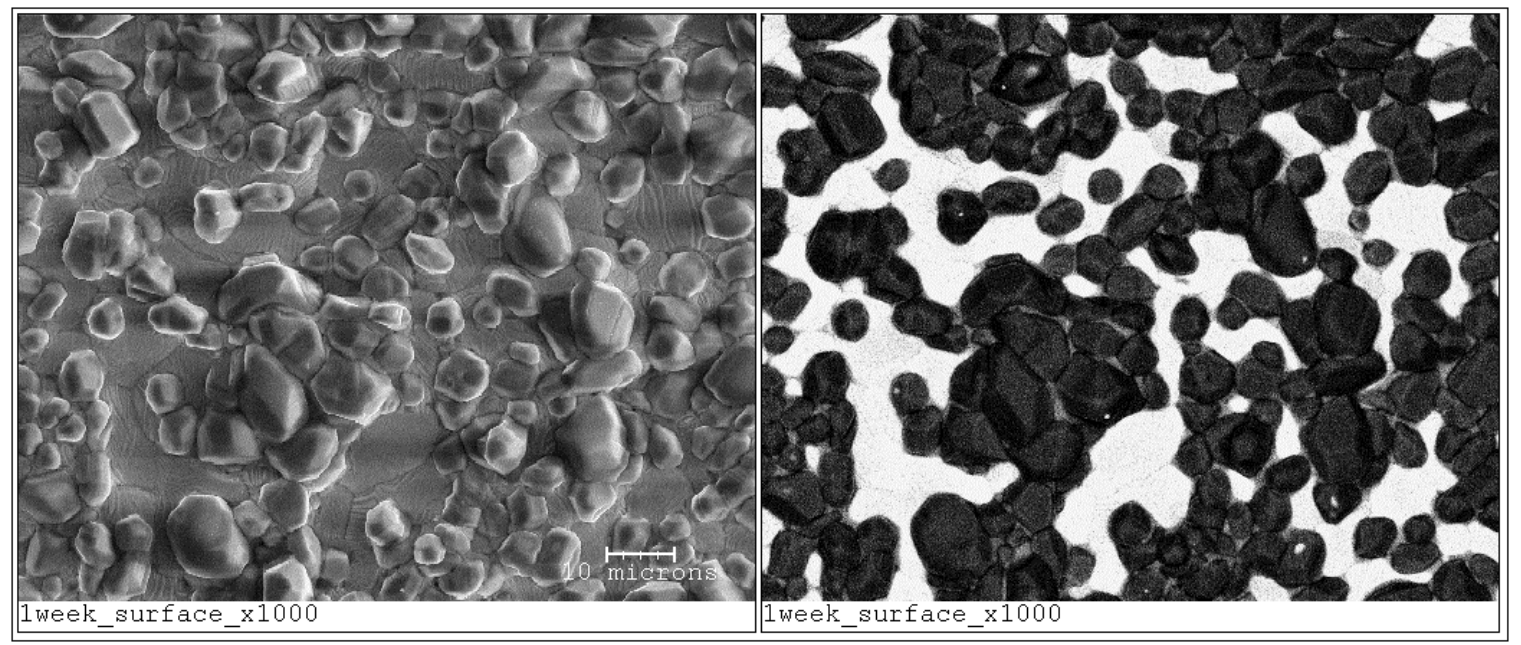

Figure 22: Sterling silver surface and x-ray spectrum after 1-week exposure 
There is very little noticeable change in the surface of the sterling silver sample from day three to the end of the first week of exposure. The copper-oxide is now covering approximately $72 \%$ of the sample surface. The largest particles at the surface are in the range of $\sim 10 \mu \mathrm{m}$ and the number of smaller particle is still consistent with previous images.

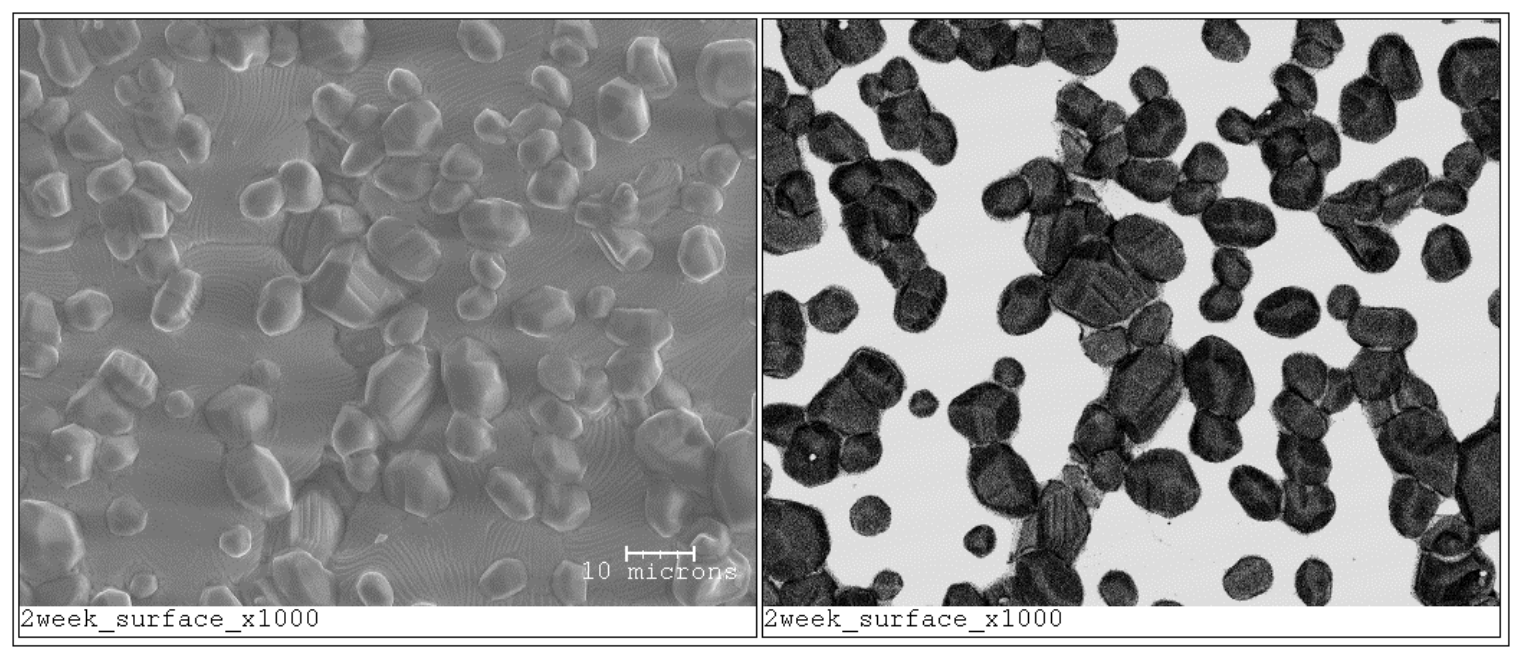

Figure 23: Sterling silver surface and x-ray spectrum after 2-week exposure

After the second week of exposure it appears that the maximum particle size has remained constant, however it does not appear that there are as many smaller particles as there had been after week one. It also appears that there is more silver exposed at the surface of the sample, approximately $55 \%$ of the surface is now covered by copper-oxide. 


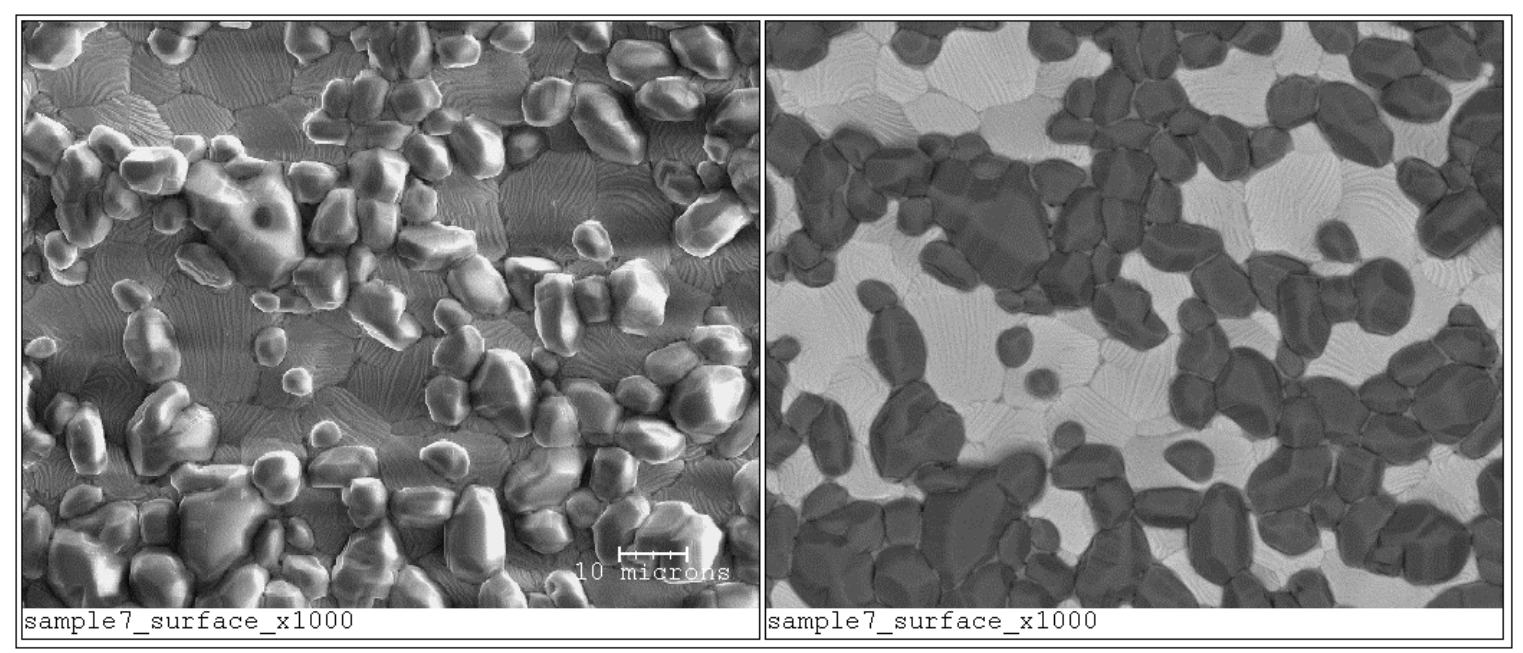

Figure 24: Sterling silver surface and x-ray spectrum after 4-week exposure

After four weeks of exposure it appears that the amount of exposed silver at the surface of the sample has remained relatively constant. Calculations found that approximately $64 \%$ of the surface was covered by copper-oxide in this sample. It does appear that more copper-oxide particles are visible, however they appear to be more crowded together than in earlier weeks of the experiment.

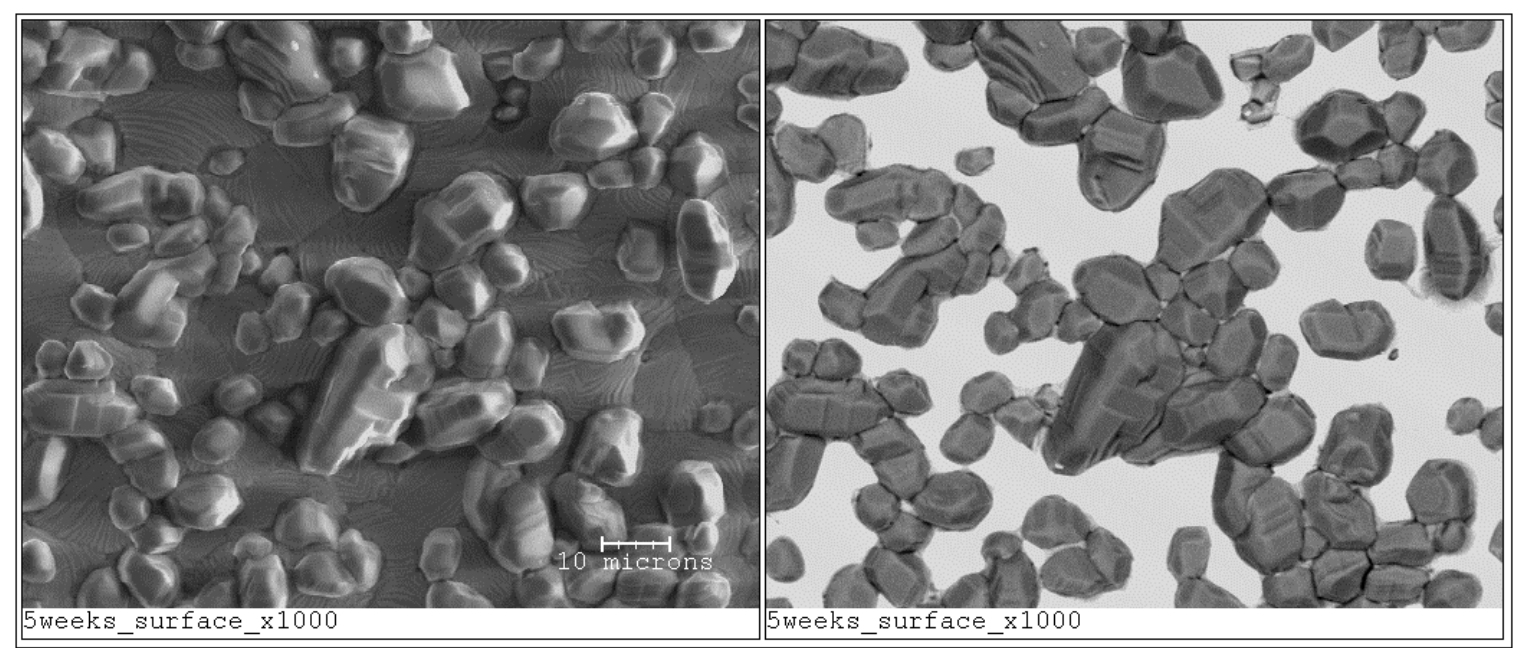

Figure 25: Sterling silver surface and x-ray spectrum after 5-week exposure 
After five weeks of exposure it appears that the maximum particle size has not changed much, however it does appear that there are fewer small particles than in previous weeks. The amount of exposed silver at the surface appears to be relatively consistent with previous weeks, based on calculations the surface of the sample has approximately $61 \%$ copper-oxide coverage.

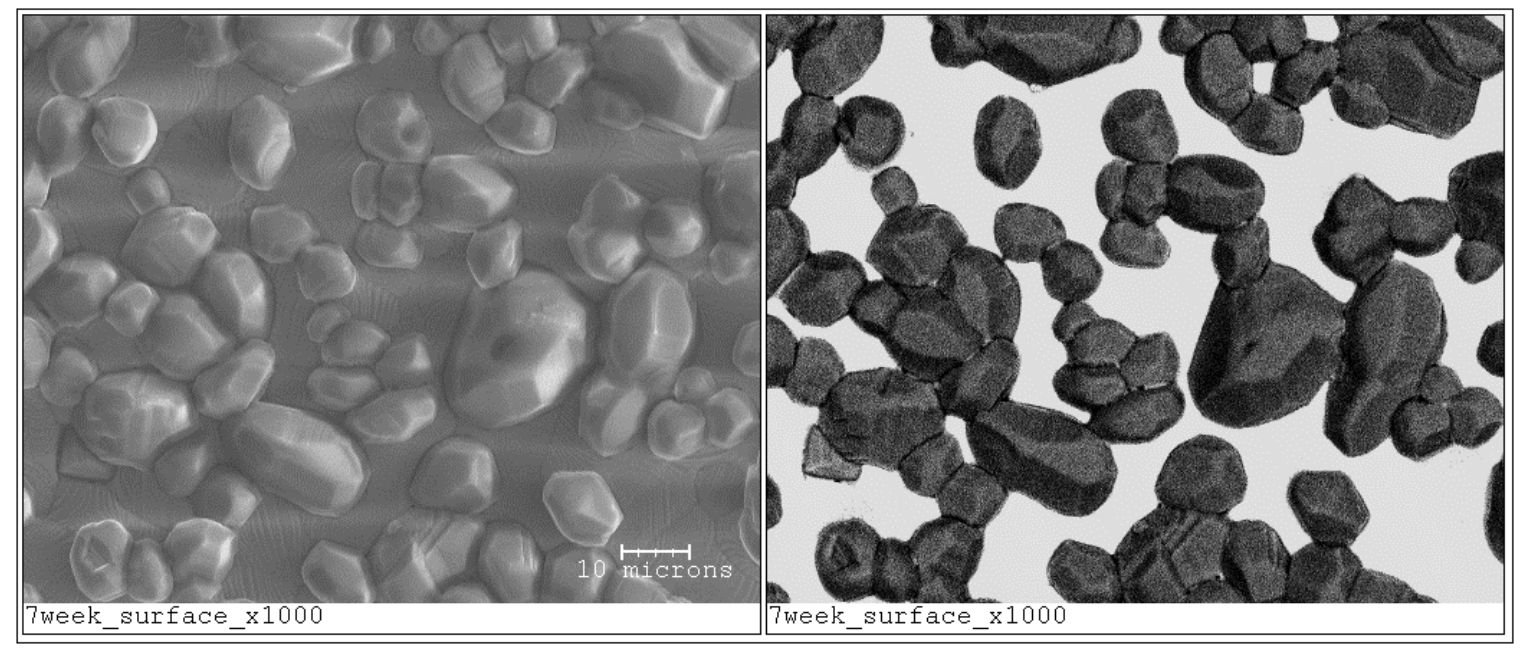

Figure 26: Sterling silver surface and x-ray spectrum after 7-week exposure

As there was little noticeable change in the particle surface from week to week at this point, samples were exposed for longer durations before examination. After seven weeks of exposure large particles have begun to dominate the surface of the sample. The surface is covered by $63 \%$ copper-oxide. It appears that in these first several weeks of exposure, the copper-oxide particles are going through a process of migration and agglomeration, which can be seen as larger and larger particles are dominating the surface of the sample and the percent of surface covered by copper-oxide decreases. 


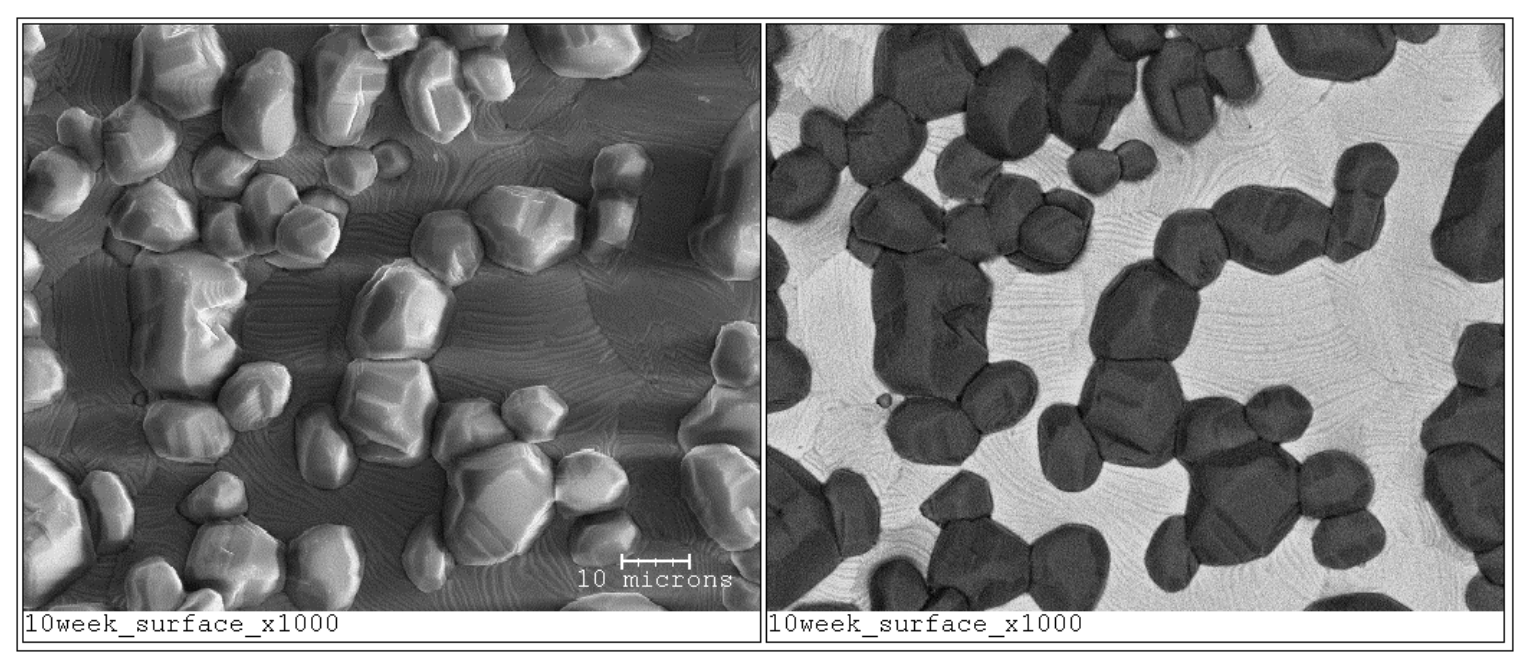

Figure 27: Sterling silver surface and x-ray spectrum after 10-week exposure

After ten weeks of exposure, it appears that the amount of silver exposed at the surface of the sample has increased as very few small particles remain. The maximum particle size of the particles at the surface has also increased as some of the particles can be seen to be approaching $20 \mu \mathrm{m}$ at this point. This change in surface microstructure is undesirable as the evaporation rate of the silver will increase with greater exposure. At this point the surface coverage of copper-oxide was calculated to be roughly $56 \%$. 


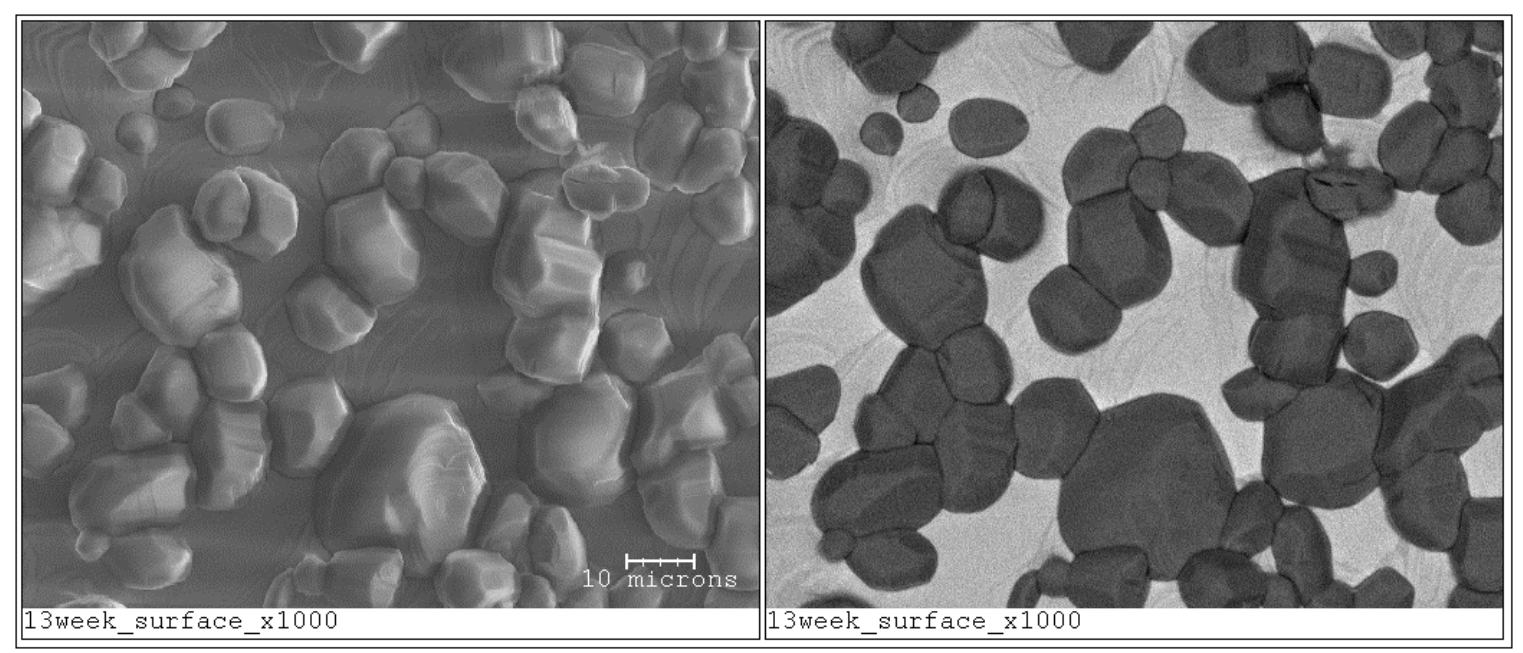

Figure 28: Sterling silver surface and x-ray spectrum after 13-week exposure

After 13 weeks the sample surface does not show much significant variation. The surface copper-oxide coverage is roughly $67 \%$. It appears that the distribution of small copper-oxide particles and larger particles is consistent with previous weeks.

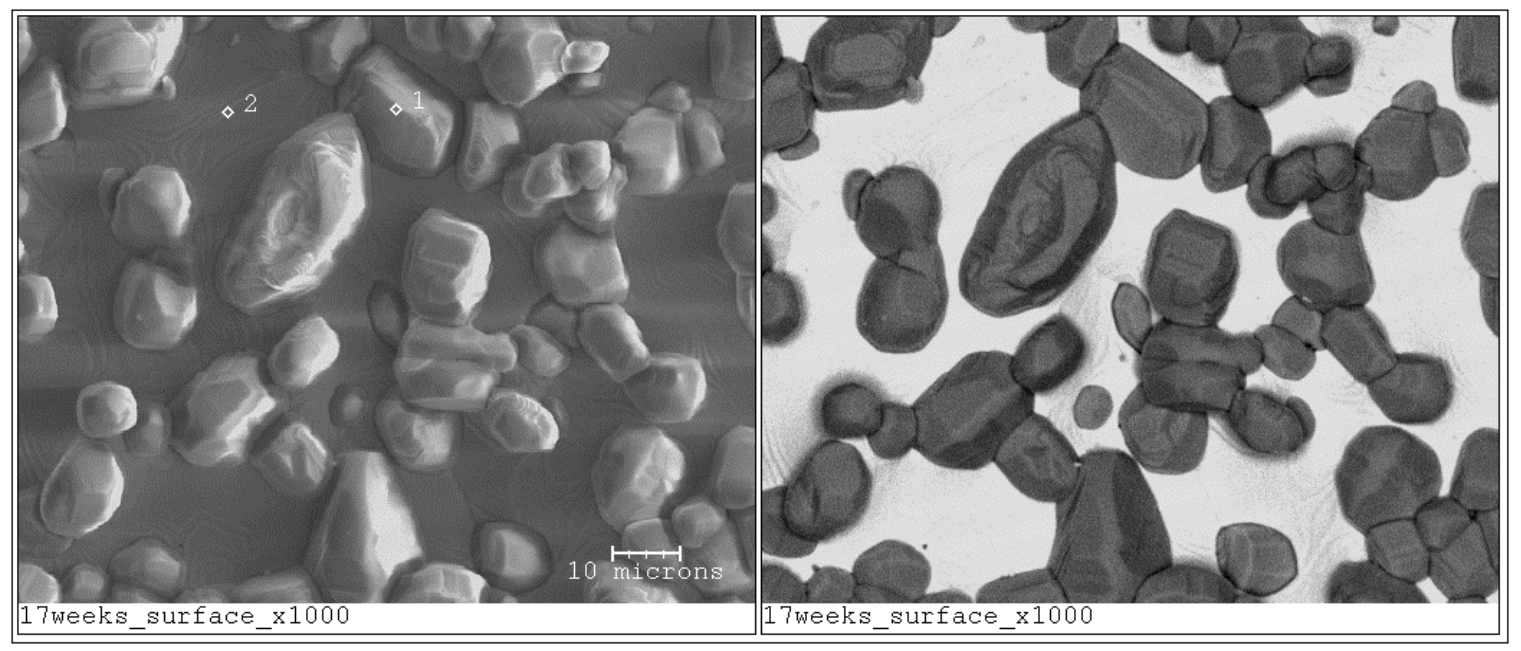

Figure 29: Sterling silver surface and x-ray spectrum after 17-week exposure

The copper-oxide coverage is roughly $61 \%$ after 17 weeks of high-temperature exposure, in-line with most of the results from previous weeks. 


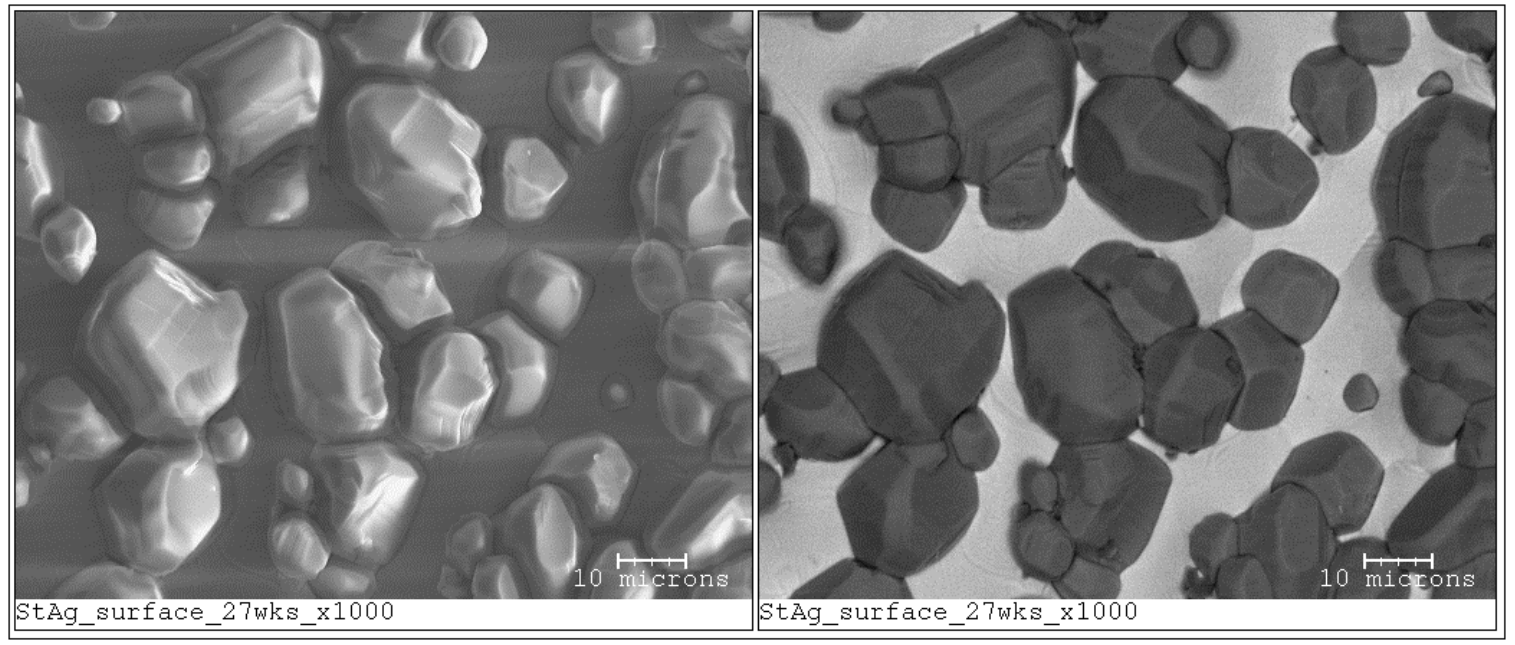

Figure 30: Sterling silver surface and x-ray spectrum after 27-week exposure

After 27 weeks of high-temperature exposure it appears that the surface of the sterling silver sample has experienced further coarsening of the copper-oxide particles. At this point, the surface can be seen to be dominated by very large particles, sometimes 20 microns across. The actual copper-oxide coverage at this point is about $70 \%$. This increase in coverage is likely due to the evaporating silver uncovering copper-oxide that would not have been visible in previous weeks.

It appears that after the first week of high-temperature exposure, there is not much variation in the sample surface regarding copper-oxide coverage. The percent coverage for the sample did show variation from $55 \%$ to $70 \%$. The range of copper-oxide coverage appears reasonable considering the number of samples and duration of the experiment. 


\subsection{FRACTURE-SURFACE ANALYSIS}

The fracture-surface of several of the samples was also evaluated to try and determine the thickness of the copper-oxide visible on the surface as well as examine copper-oxide particles below the surface of the sample.

The samples cross-sections were prepared by immersing the samples in liquid nitrogen and then breaking the frozen sample in half. This technique was employed to avoid possible smearing of silver that can occur during polishing which can distort the image.

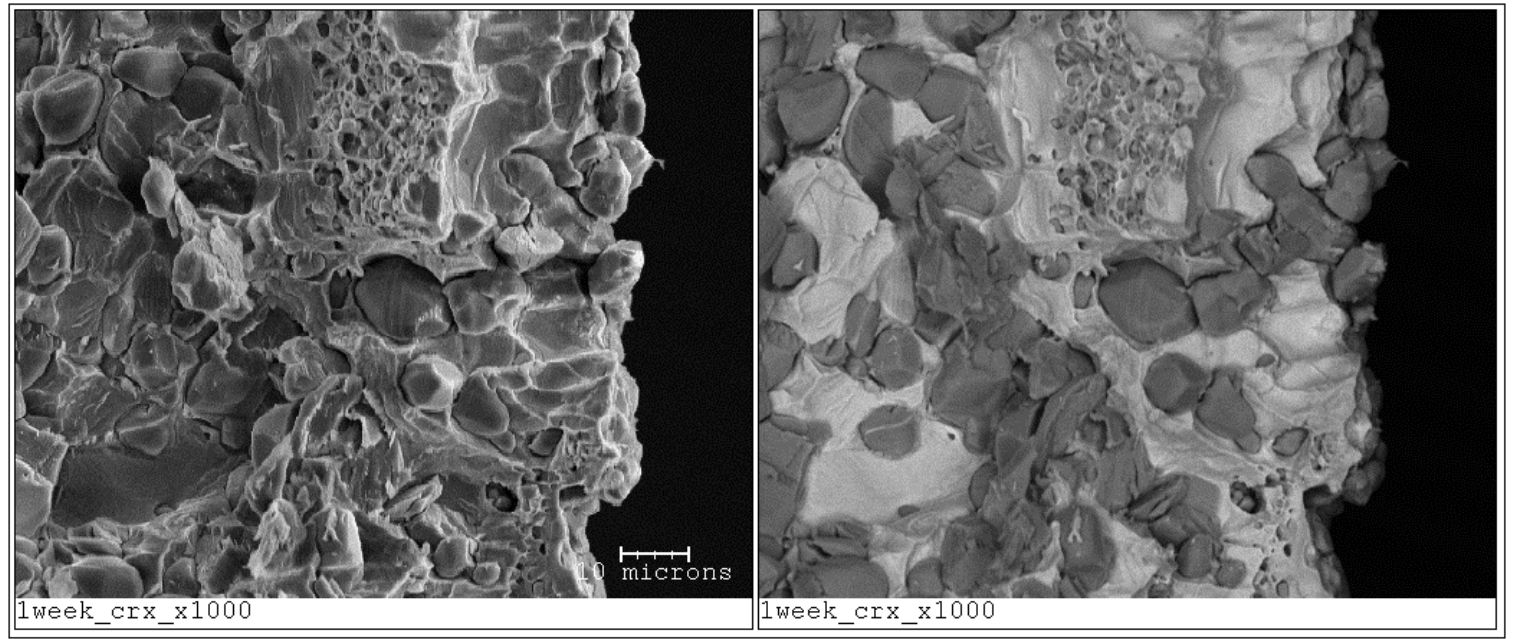

Figure 31: SEM micrograph and backscatter of sterling silver cross-section (1-week exposure) 

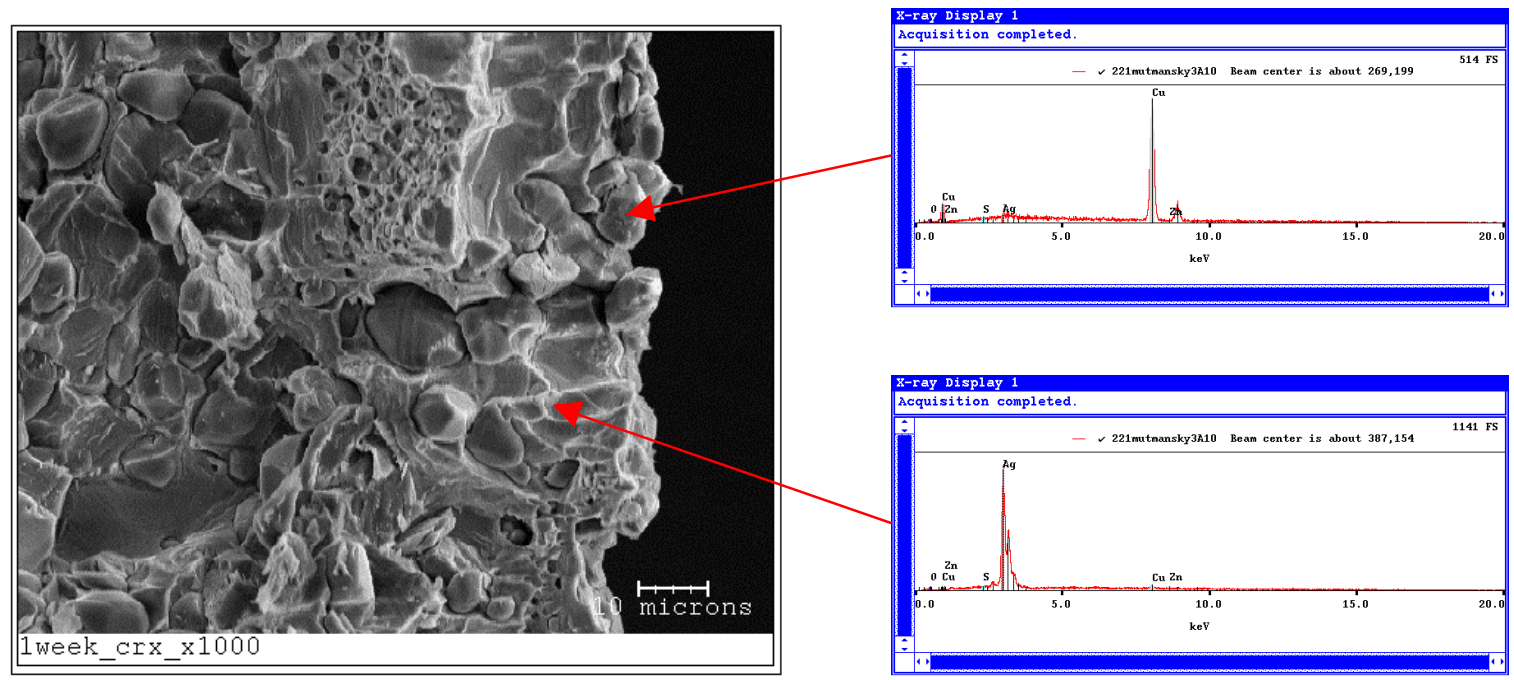

Figure 32: SEM micrograph and x-ray spectrum of sterling silver cross-section (1-week exposure)

In this cross-sectioned image of the sterling silver sample the surface layer can be seen at the right-hand edge of the image. The x-ray spectrum confirms the presence of copper-oxide at the surface of the sample. It can be seen that there is also an abundance of copper-oxide particles below the surface of the sample, many of which are much larger than the surface particles. These internal particles could serve an important role in terms of inhibiting the evaporation of silver during a very long-term exposure of the sample.

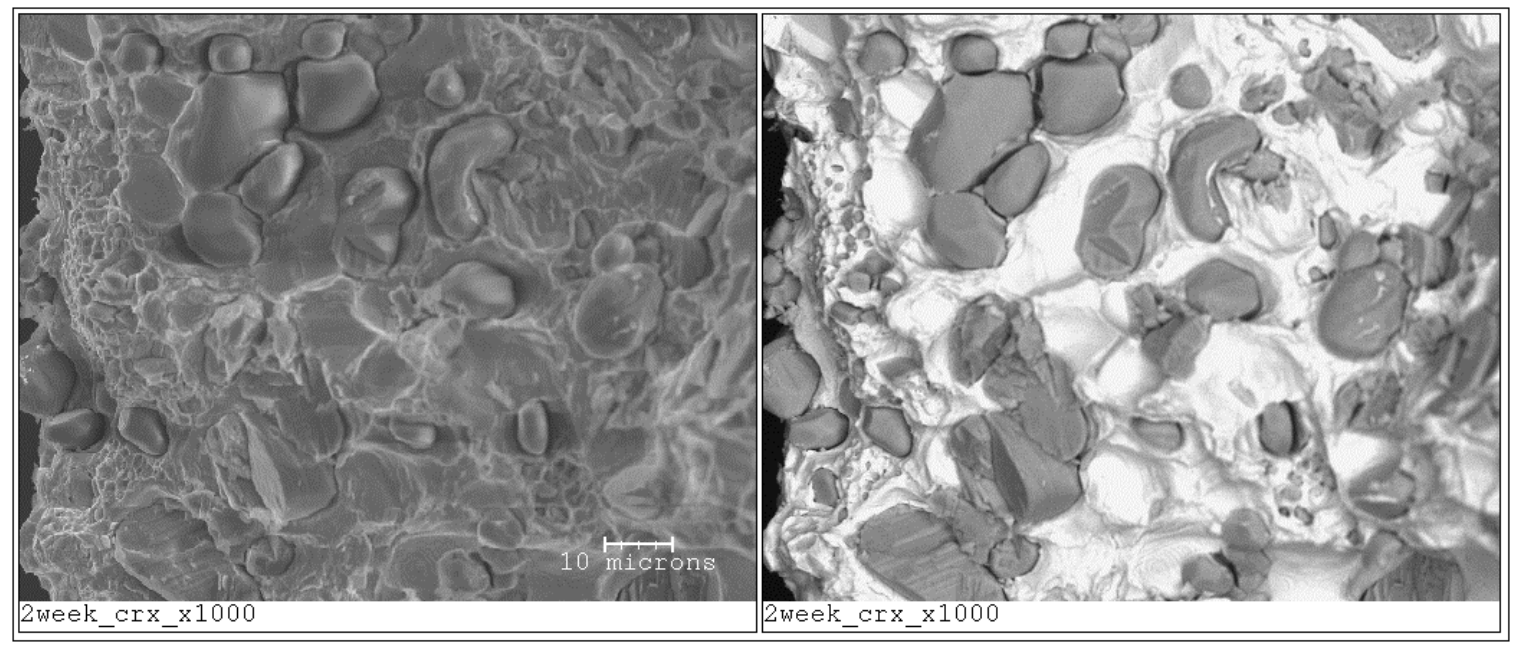

Figure 33: SEM micrograph and backscatter of sterling silver cross-section (2-week exposure) 
After two weeks of exposure large internal copper-oxide particles can still be seen in the sample. The surface of the sample shows no observable difference in terms of silver recession from that of the sample under exposure for one week.

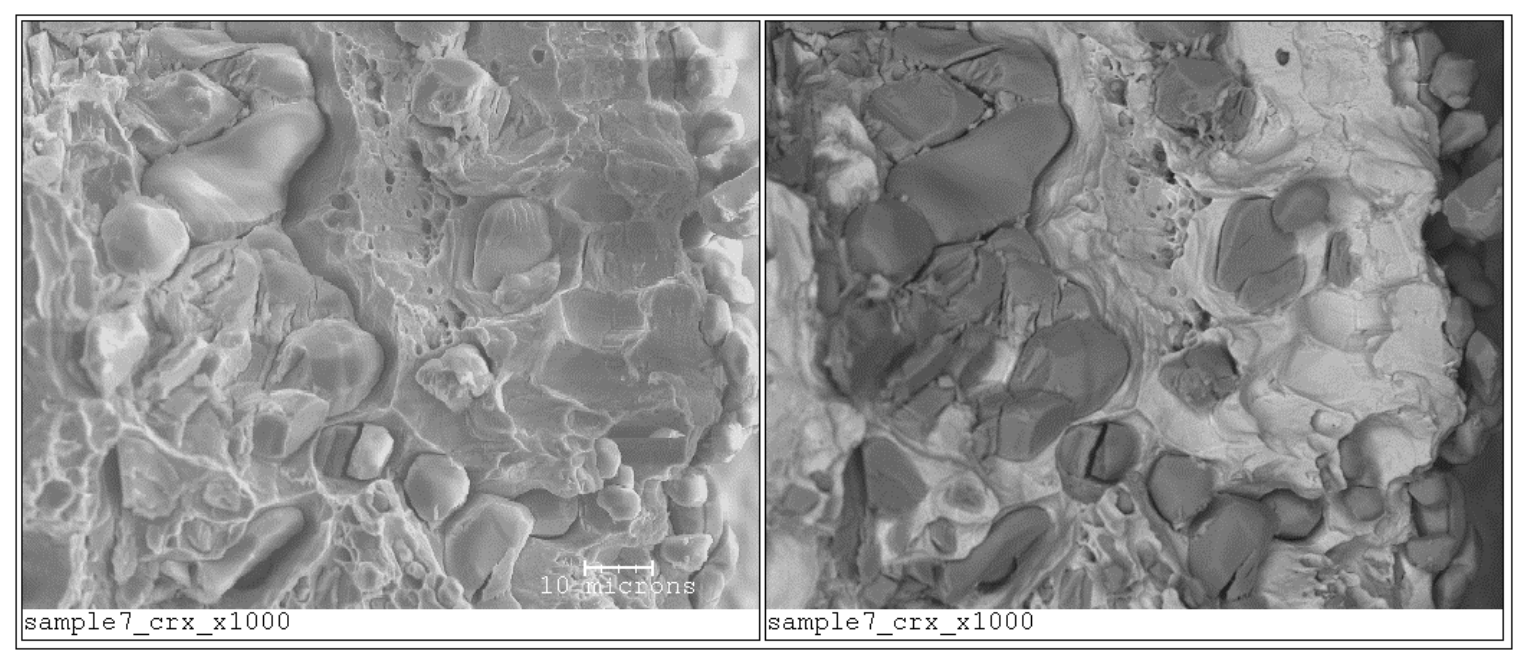

Figure 34: SEM micrograph and backscatter of sterling silver cross-section (4-week exposure)

After four weeks of exposure there is still no noticeable difference in the sample in regards to silver recession when viewed in cross-section, however large internal particles can still be seen approximately $20 \mu \mathrm{m}$ below the surface. 


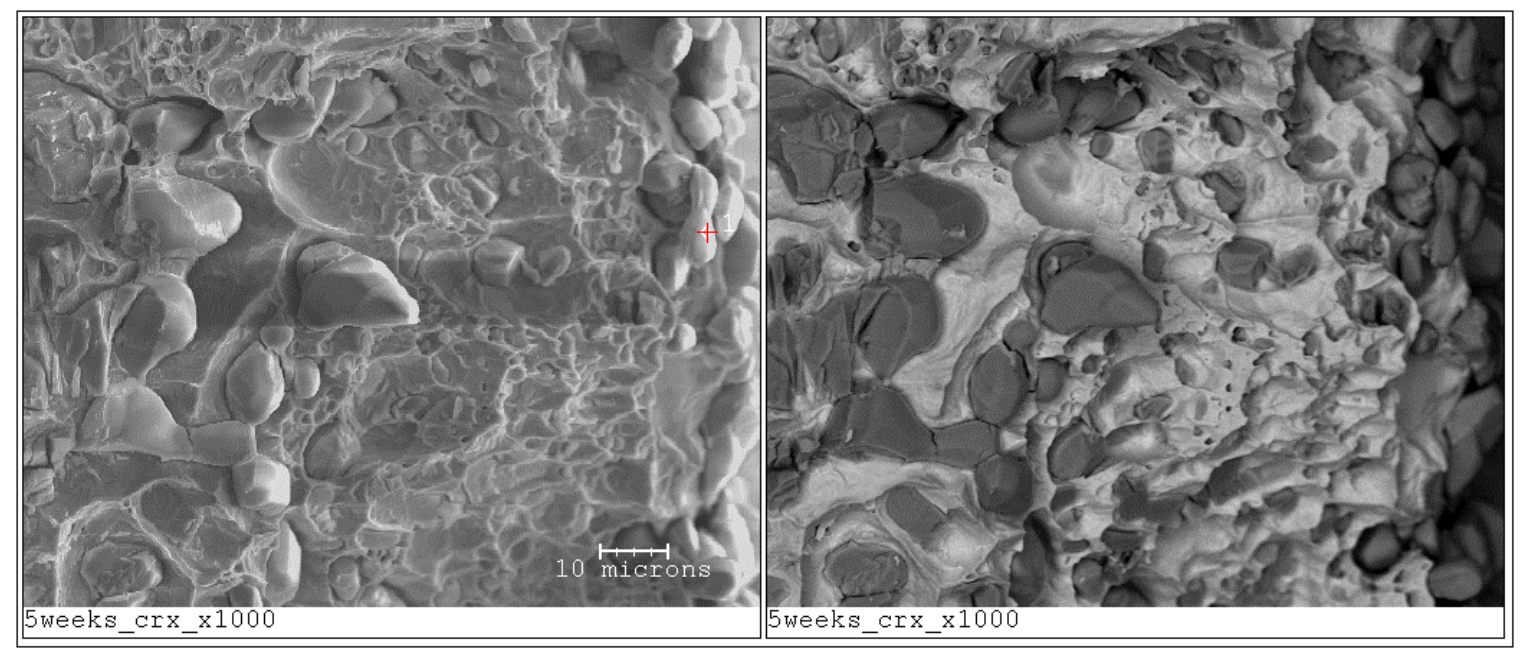

Figure 35: SEM micrograph and backscatter of sterling silver cross-section (5-week exposure)

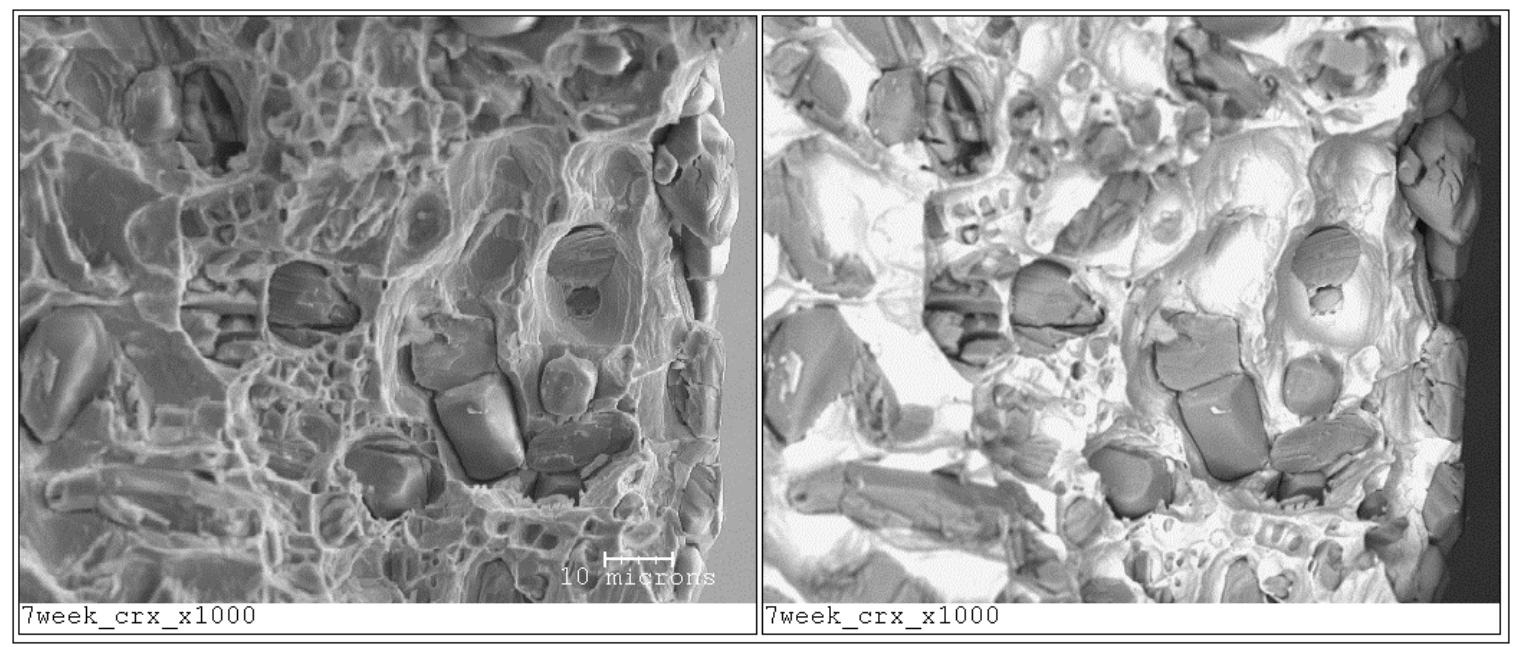

Figure 36: SEM micrograph and backscatter of sterling silver cross-section (7-week exposure)

It is evident from the surface images that the copper-oxide layer does not cover the entire sample, however, it appears that the thickness of the layer remains relatively constant soon after exposure. It also appears that the internal copper-oxide particle size remains relatively constant from week to week. From the cross-section imaging it does not appear that there is significant loss of silver deep inside the samples. 


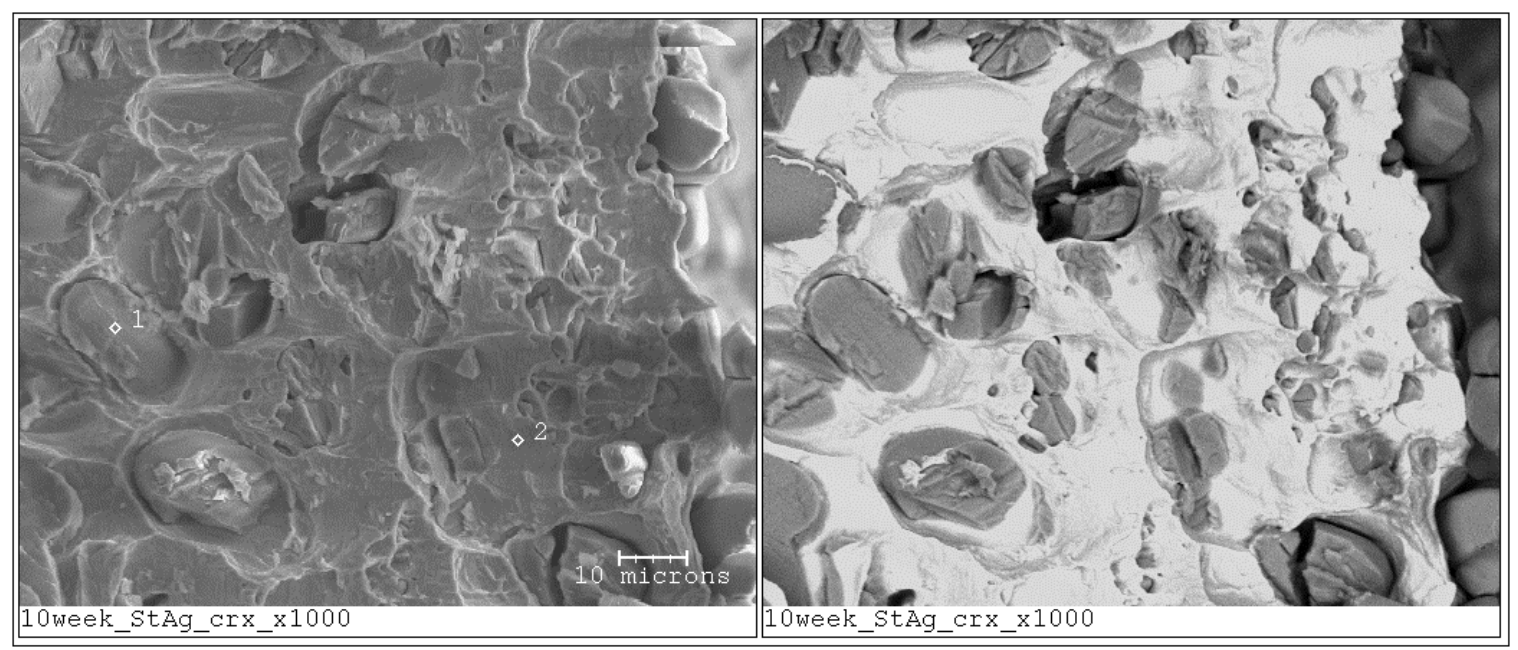

Figure 37: SEM micrograph and backscatter of sterling silver cross-section (10-week exposure)

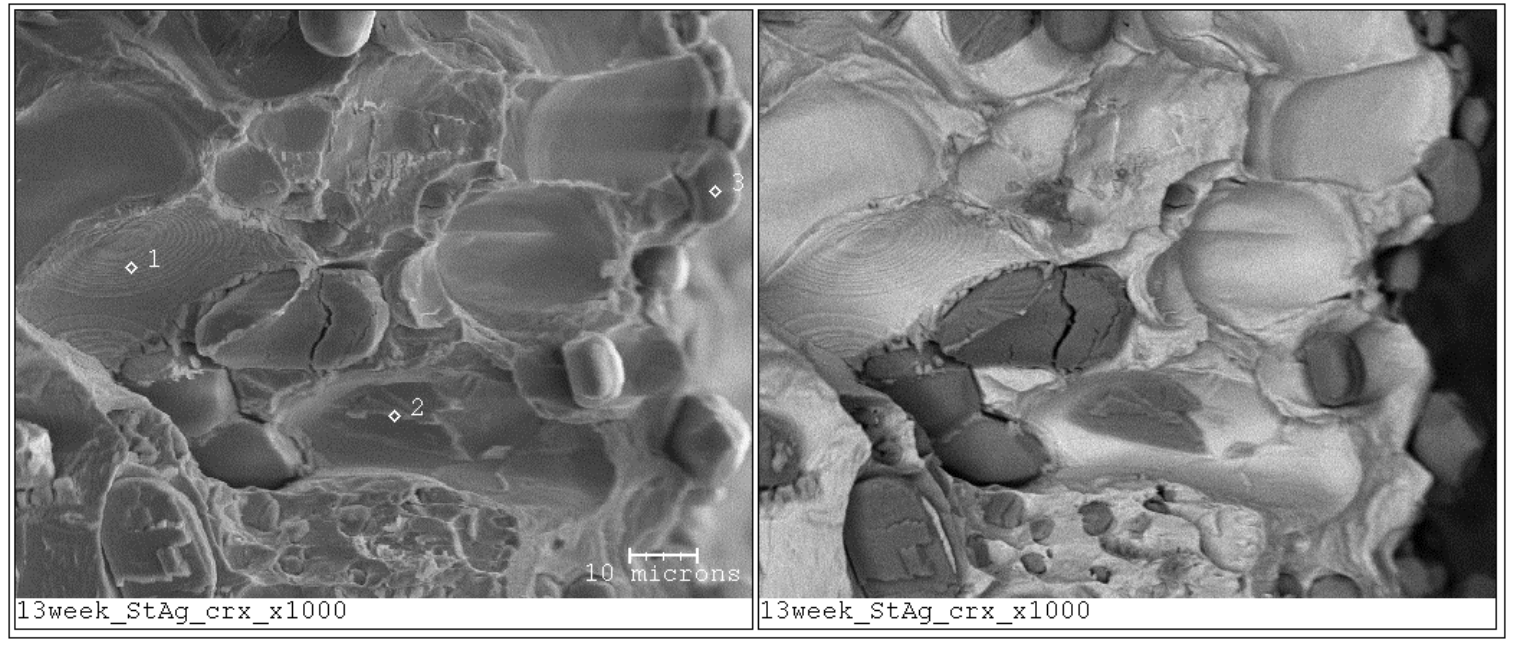

Figure 38: SEM micrograph and backscatter of sterling silver cross-section (13-week exposure) 


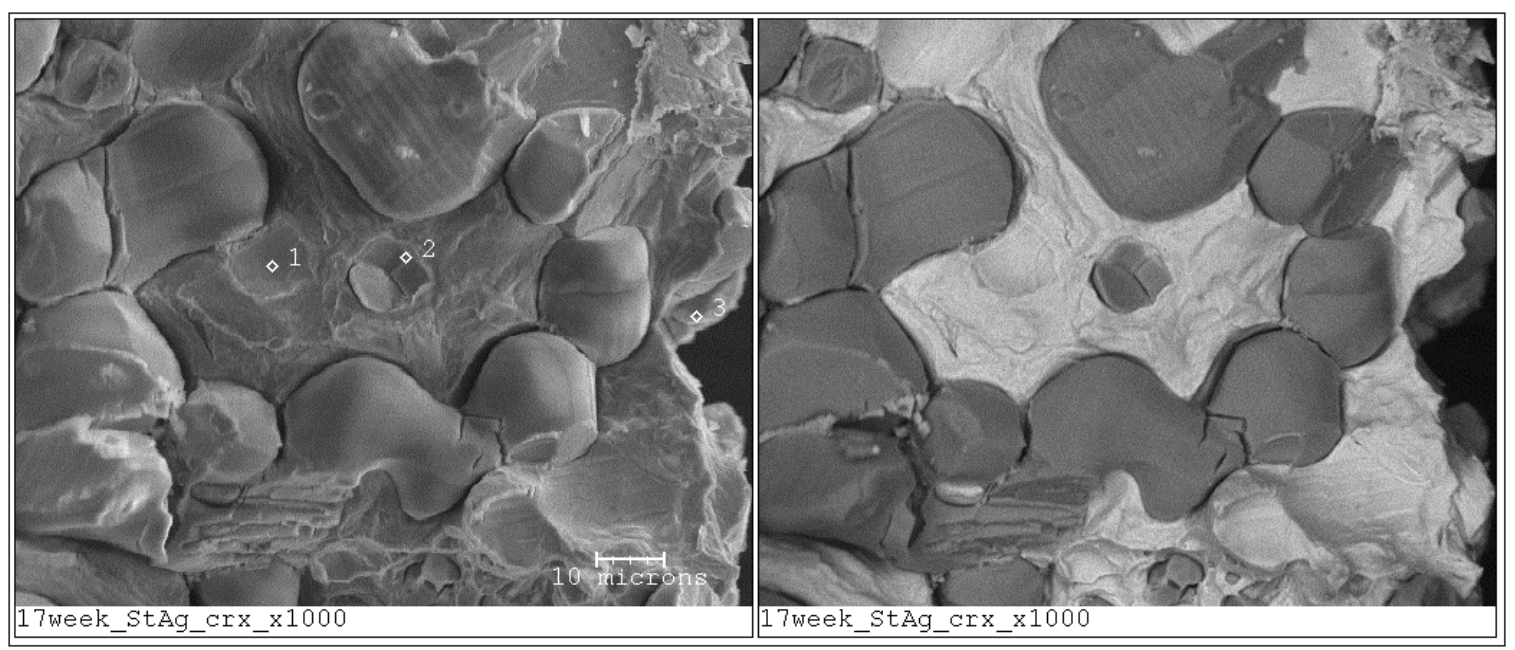

Figure 39: micrograph and backscatter of sterling silver cross-section (17-week exposure)

In the last several weeks of exposure there does not appear to be much difference in the copper-oxide particles at the surface with regards to thickness of the surface layer. It does appear that particle below the surface have become larger than seen in previous weeks, possibly due to agglomeration or coarsening.

\subsection{STERLING SILVER LOSING MASS MORE RAPIDLY THAN PURE SILVER}

After several weeks of exposure in the tube furnace it became evident, based on the loss of silver to evaporation, that the sterling silver samples were actually evaporating more rapidly than their pure silver counterparts. Initially it was thought that some of the mass loss of the sterling silver samples may be due to a loss of copper-oxide particles at the surface. A simple experiment was carried out to verify this. 


\subsubsection{Compressed Air Experiment}

When observing the changing surface microstructure of the sterling samples from over several weeks it appeared that the number of copper-oxide particles, particularly the smaller particles was appearing to decrease. This reduction in particles could be due to a number of factors. Possibly the smaller particles have grown together into the larger particles that remained visible on the surface, or possibly the smaller particles were carried away in the air. A simple experiment was conducted to test if the particles could be blown off of the surface.

Samples were marked so as to be imaged using SEM at a particular point. Afterward the sample was sprayed with a can of compressed air at room temperature and returned to the SEM for analysis at the original mark to see if any particles had been blown away. This procedure was carried out for a sample with abundant small particles (3-day oxidation) as well as a sample with fewer small particles due to longer exposure (10 week oxidation), the idea being that the silver had receded to the extent that the few remaining small particles would be easier to remove. The SEM imaging for these tests is shown in the following figure. 


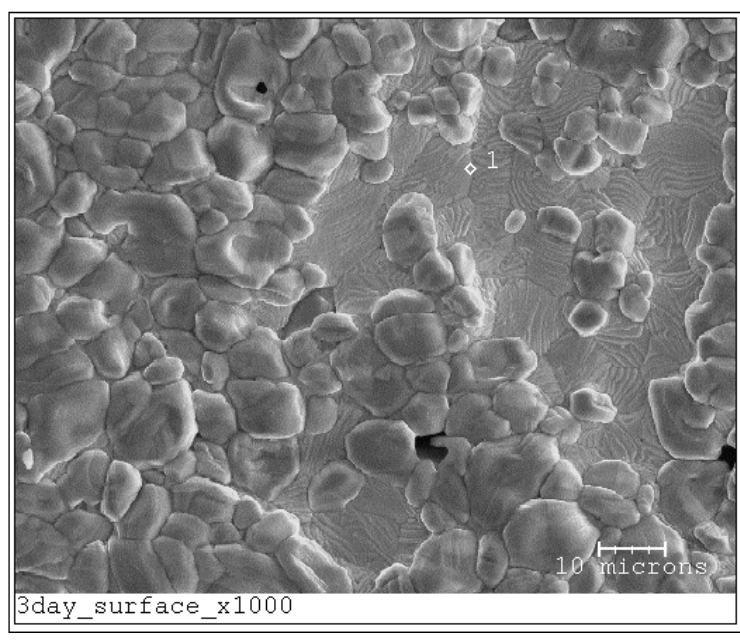

nกTกก

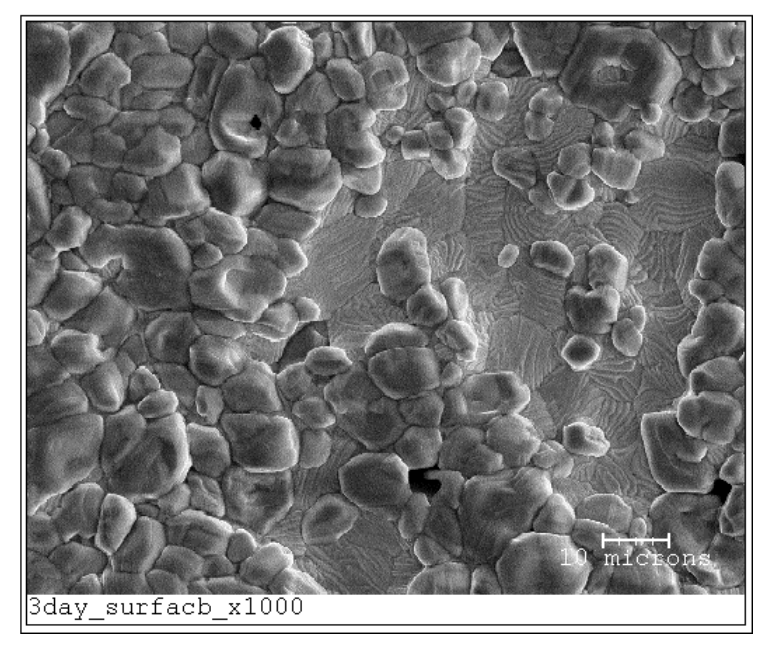

A птกก

Figure 40: SEM micrographs of sterling silver surface before and after exposure to compressed air (3-day sample)

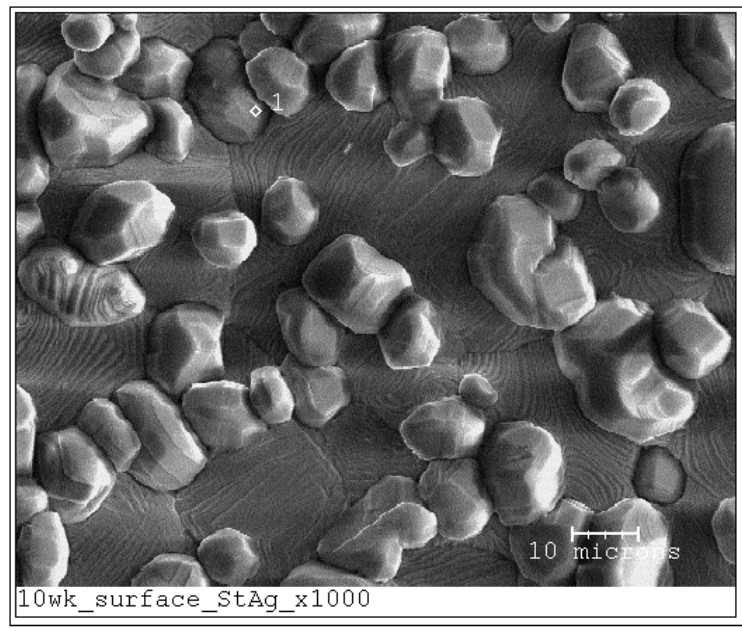

BEFORE

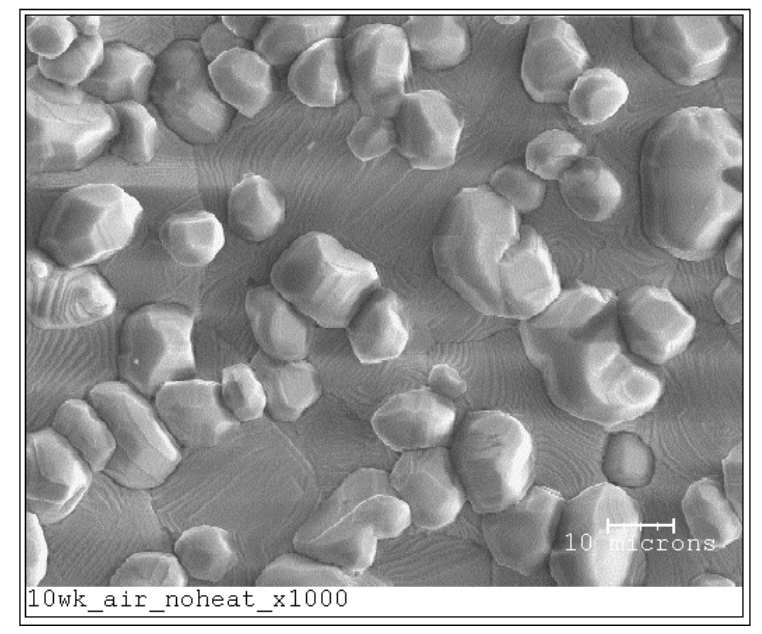

AFTER

Figure 41: SEM micrographs of sterling silver surface before and after exposure to compressed air (10-week sample)

In both cases the surface after exposure appears identical to the pre-exposed surface indicating that copper-oxide particles are not being carried away in the air flow. This may indicate that copper-oxide particles are not actually lost but simply migrate and 
agglomerate, making it appear that some of the particles are being lost from week to week.

\subsection{SEM ANALYSIS: PURE SILVER SAMPLES}

Pure silver samples were also exposed in the tube furnace under the same conditions as the sterling silver samples. The pure silver samples were simply purchased in sheets and cut to size. The samples dimensions were measured and their initial masses were determined using an electronic scale, the samples were then cleaned using acetone. Their masses were tracked from week to week and their corresponding thickness changes were calculated. A pure silver sample was also imaged using SEM weekly to evaluate changes in microstructure. The SEM of the pure silver samples are shown in the following figures:

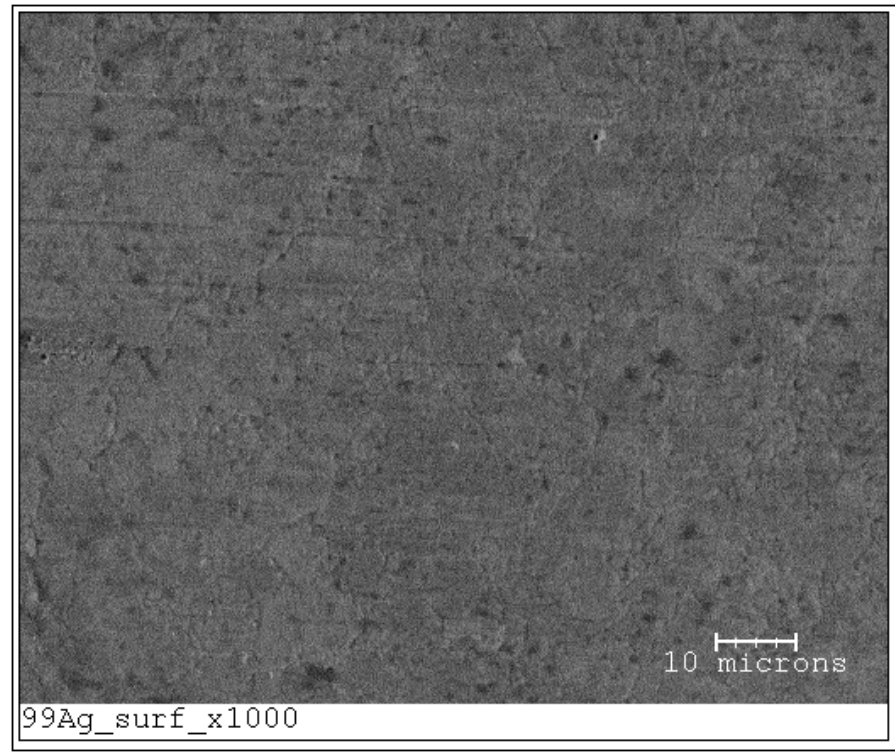

Figure 42: SEM micrograph of pure silver surface (no exposure) 


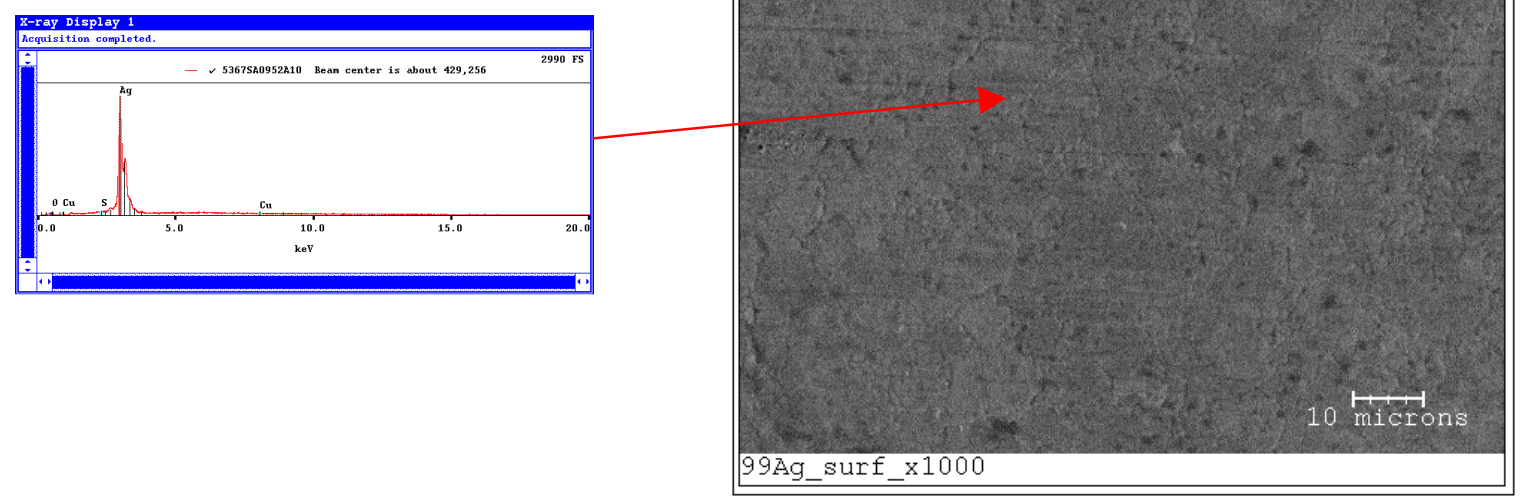

Figure 43: Pure silver (no exposure), SEM image and x-ray spectrum

The sample does not seem to show any unusual features at the surface. X-ray analysis showed only silver at the surface, no other elements were found.

The following series of SEM micrographs illustrate the changing surface of the pure silver surface throughout the duration of their high-temperature exposure. The full size images are shown in the appendix. 


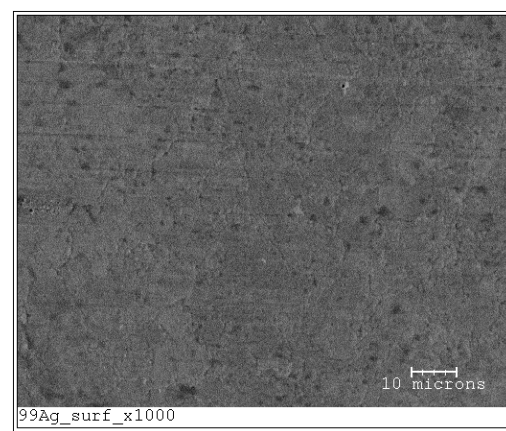

Pre-exposed
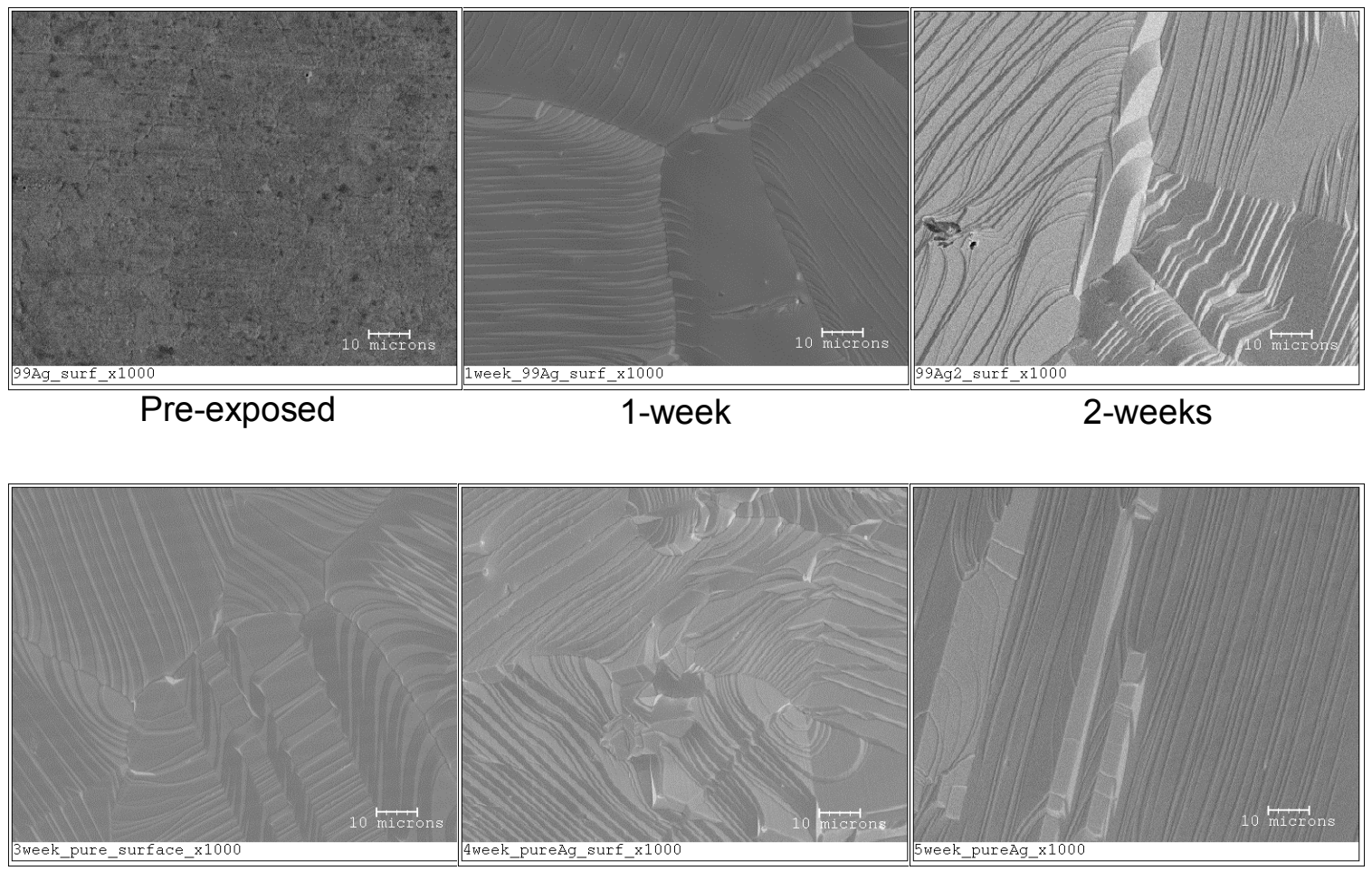

3-weeks

4-weeks

5-weeks
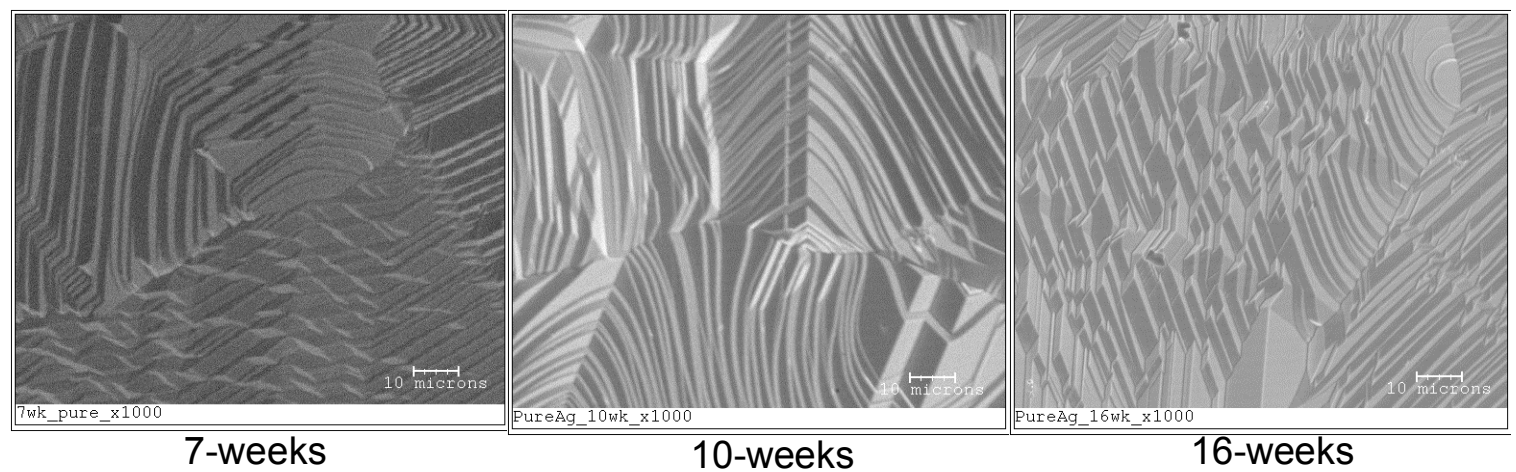

7-weeks 10-weeks 16-weeks

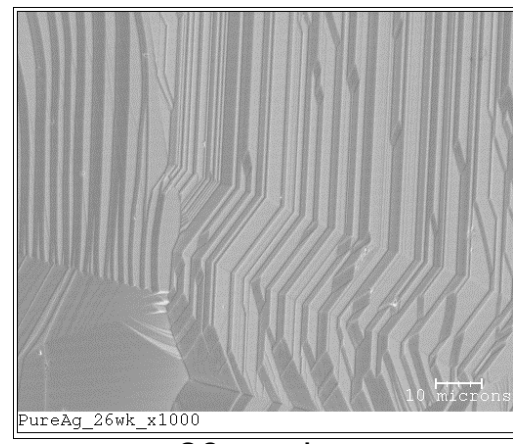

26-weeks

Figure 44: Pure silver surfaces for high-temperature exposure 
After one week, the only microstructure changes evident at the surface of the sample is faceting. At this point, the faceting does not appear very dramatic.

After two weeks the faceting on the pure silver sample has become more pronounced than previously. At this point, the collected data was indicating that the pure silver sample was losing mass more slowly than the sterling silver sample. It is likely that through continued faceting, the silver is able to reach an orientation that is less favorable to evaporation and thus causes a reduction in the rate of mass loss and thickness reduction of the sample.

Over the course of the experiment the only very clear change that can be seen in the surface of the pure silver samples is faceting. There does appear to be an increase in the amount of steps visible in later weeks of the experiment, but it does not appear from the SEM micrographs that the faceting is any more pronounced after the second week of high-temperature exposure. It appears that faceting is much more pronounced in the pure silver samples than in the sterling samples. It is possible that the copper-oxide particles inhibit faceting of the silver in the sterling samples and allow them to maintain a higher rate of evaporation, because the silver is never able to reach the orientation least favorable to evaporation. 
CHAPTER 5: Time-series analysis of sterling silver samples

Time-series analysis of the sterling sample involves examining the same area of a sample using SEM repeatedly over the course of long-term high-temperature exposure in order to better understand microstructure changes taking place at the surface.

\section{1 TIME-SERIES ANALYSIS: EARLIER STAGE STERLING SAMPLE}

These samples were pre-marked so that the area of interest could be relocated in the SEM. The samples were imaged every two weeks in order to allow noticeable changes to occur to assist in the analysis.

Before SEM analysis began, the sample was oxidized in a box furnace for twelve hours in order to get good copper-oxide coverage at the surface. The time-series of images is illustrated in the following figure. All images are backscatter to help differentiate copper-oxide particles from surrounding silver. The SEM micrographs and backscatter images are shown in the appendix in larger format. 

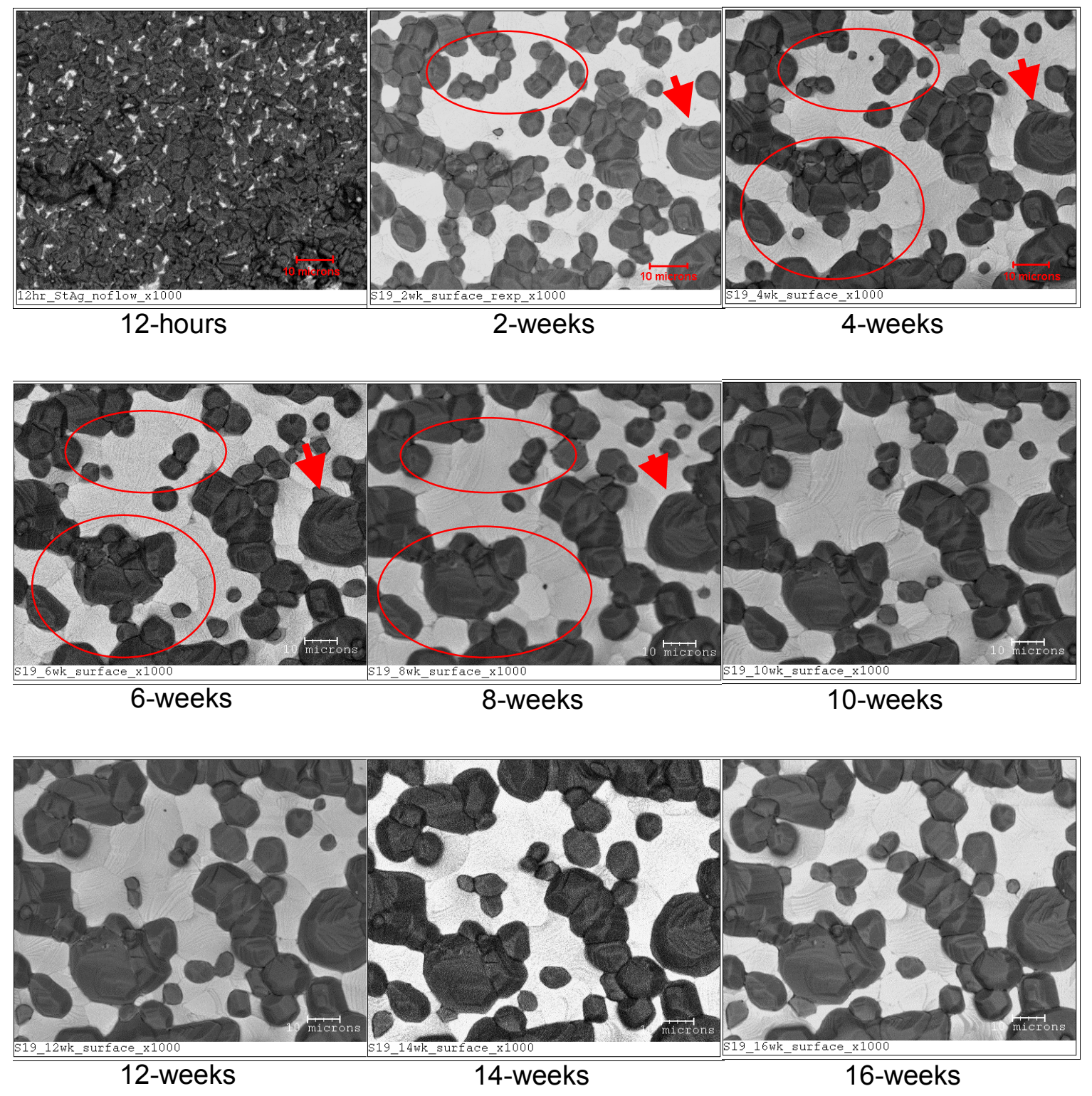

Figure 45: Time-series analysis for early-stage sterling sample, low-magnification

After the initial period of oxidation in the box furnace, the sample surface can be seen to be almost completely covered by copper-oxide. After oxidation in the box furnace the copper-oxide coverage at the surface is calculated to be $\sim 94 \%$. 
After two weeks of high-temperature exposure, it is clear that the copper-oxide has coarsened into much larger surface particles than were visible after just 12-hours of oxidation. Also, much more silver is also exposed at the surface of the sample, which may be an undesirable effect. After two weeks the copper-oxide coverage at the surface is calculated to be $\sim 59 \%$.

Through continued high-temperature exposure of the sample the major surface changes are migration and agglomeration of the surface copper-oxide particles. The group of particles in the lower right of the images can be seen to agglomerate from a group of small particles into a single much larger particle. The large particle on the righthand side of the image can also be seen to increase in size, likely due to a combination of particle coarsening and recession of the silver at the edges of the particle. The calculated surface copper-oxide coverage is $\sim 62 \%$ by week 16 of exposure. The increase in copperoxide coverage is likely indicative of silver recession exposing slightly more of the copper-oxide particles during exposure, making it more visible at the surface.

Over the course of the experiment it appears that the copper-oxide particles continue to coarsen. It also appears that other particles begin to appear, most likely due to continued silver recession. Over time it is likely that these newly exposed particles will migrate and agglomerate with other surface particles.

Higher magnification images of the sample surface were also used to for timeseries analysis. The higher magnification images focus on the upper-left corner of the images shown in the previous figures. These images illustrate the faceting of the silver surrounding the copper-oxide particles as well as offer a closer look at the interface 
between silver and copper-oxide at the sample surface. The SEM micrographs and backscatter images are shown in a larger format in the appendices.
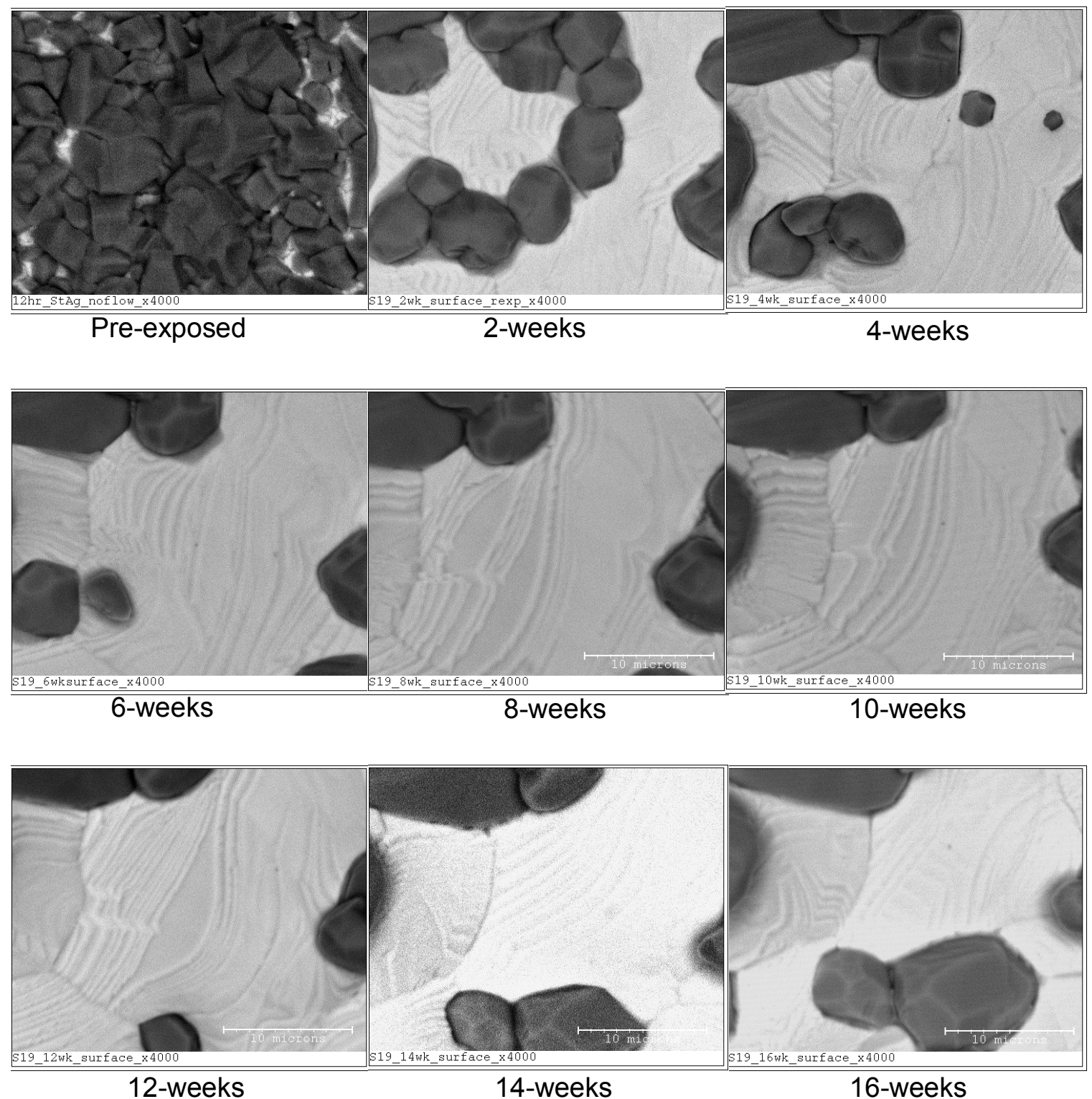

Figure 46: Time-series analysis for early stage sterling sample, High-magnification

This series of images illustrates how copper-oxide particles can disappear from some areas of the surface and be replaced by new particles. The particles initially visible 
likely disappeared due to migration and agglomeration with surrounding particles. Copper-oxide particle appear at the lower section of the observed area in later stage of the experiment. These particles appear to have been below the surface and were exposed due to evaporation of the silver above them.

It can also be seen that the faceting of the silver between the copper-oxide particles does not appear to undergo any significant changes throughout the experiment, possibly due to the presence of the copper-oxide.

\subsection{TIME-SERIES ANALYSIS: LATER STAGE STERLING SAMPLE}

A second sample was also examined using the same procedure. Instead of starting with a 12-hour oxidation, the sample used in this analysis had been exposed in the cathode environment for five weeks prior to this experiment. This allows for analysis in later stages of exposure to be examined more expediently. This analysis also includes SEM micrographs and backscatter imaging to aid in detection of discrepancies between the images. 

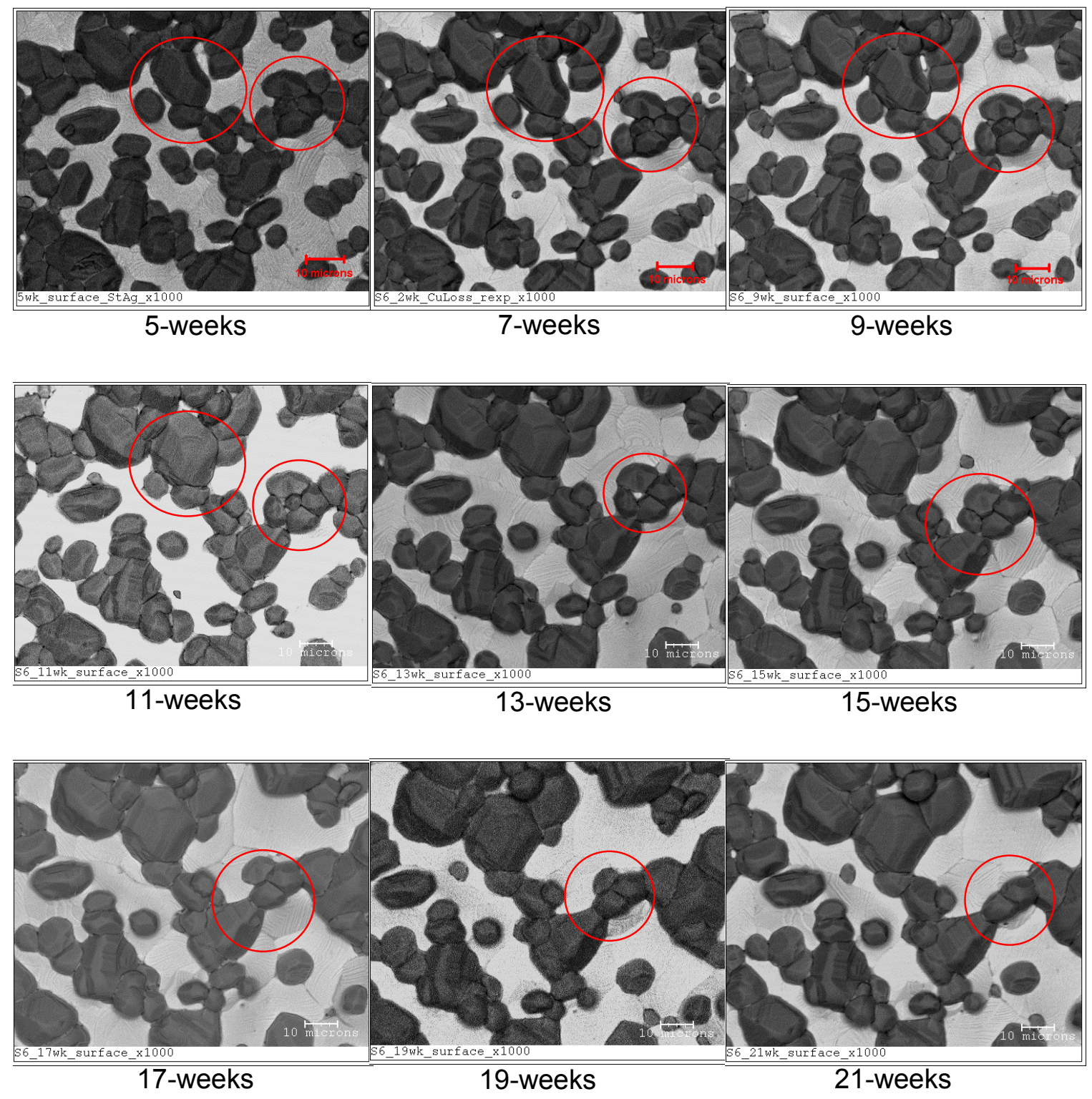

Figure 47: Time-series analysis for later sterling silver sample, low-magnification

Initially the sample is similar to other exposed samples with copper-oxide particles of varying sizes at the surface. The calculated surface copper-oxide coverage is $\sim 65 \%$, similar to the sample used in the initial sterling silver time-series analysis.

The images illustrate how silver can disappear from the edges of a copper-oxide particle given enough time in the high-temperature environment. In the area circled, the 
silver at the edge of the large copper-oxide particle can be seen to decrease in size before disappearing completely, lending credence to the idea that silver may evaporate more rapidly at the interface between the two. Other areas illustrate copper-oxide coarsening and agglomeration. Overall, the samples surface appears to be relatively stable over the coarse of high-temperature exposure, without dramatic changes on the sample surface. When calculating the copper-oxide coverage at the surface the value never changed by more that $1 \%$ in any two-week period. By week 21 the surface copper-oxide coverage is $\sim 61 \%$, a decrease over the 4-month duration of the experiment.

As in previous sample higher-magnification time-series analysis was done in order to better examine changes in silver faceting as well as the interfaces between the silver and copper-oxide particles. 

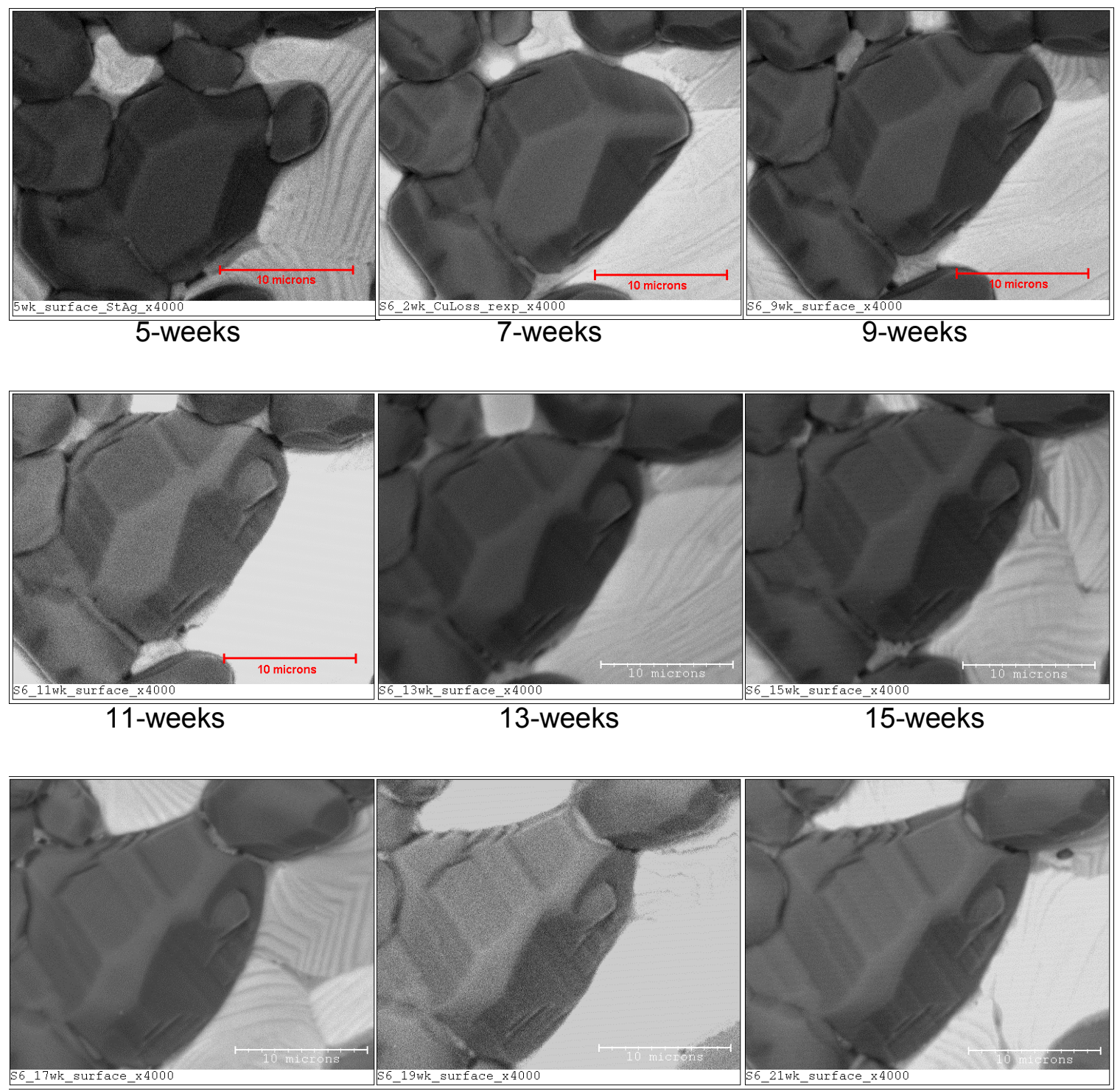

17-weeks

19-weeks

21-weeks

Figure 48: Time-series analysis for later sterling silver sample, high-magnification

From week five to week seven it can be seen that there is a dramatic change in the amount of exposure of the large copper-oxide particle at the center of the image. It appears that the silver initially surrounding the upper "nose" of the particle has receded away leaving more of the particle visible. 
Afterward, it appears that the nose of the copper-oxide particle moves into contact with the smaller copper-oxide particles above it, possible due to evaporation of the silver initially between the particles. Throughout the duration of the experiment the particles continue to move toward one another, and are appearing to agglomerate by week 21 . The silver faceting is difficult to see unfortunately due to the contrast of the images. It does appear that there is a slight difference in the faceting of the silver from the earlier stages to the later stages, however, it does not appear that the silver faceting becomes more pronounced as seen in the pure silver samples during high-temperature exposure. The images illustrate the volatility of copper-oxide under the high-temperature conditions.

\subsection{PRESSING OXIDES INTO PURE SILVER SAMPLE}

\subsection{1 $\mathrm{Ag} / \mathrm{CuO}$ Sample}

As noted earlier it is clear that the sterling silver samples are losing mass more rapidly than the pure silver samples, contrary to the original expectation. This is believed to be the result of interaction between the copper-oxide particles and the surrounding silver. In order to better examine any interactions new silver/copper-oxide samples were fabricated.

Pure silver samples were cut from a sheet of $99.9 \%$ pure silver. The samples were placed in a bench press and covered in copper-oxide powder (30-50 nm original particle size). The powder was then pressed into the surface of the silver with approximately $400 \mathrm{MPa}$ of force at room temperature. These samples were then placed in the tube furnace for high-temperature exposure. The image below illustrates the 
interface between the copper-oxide and silver in this sample. The backscatter image to the right aids in distinguishing the copper-oxide from the silver.

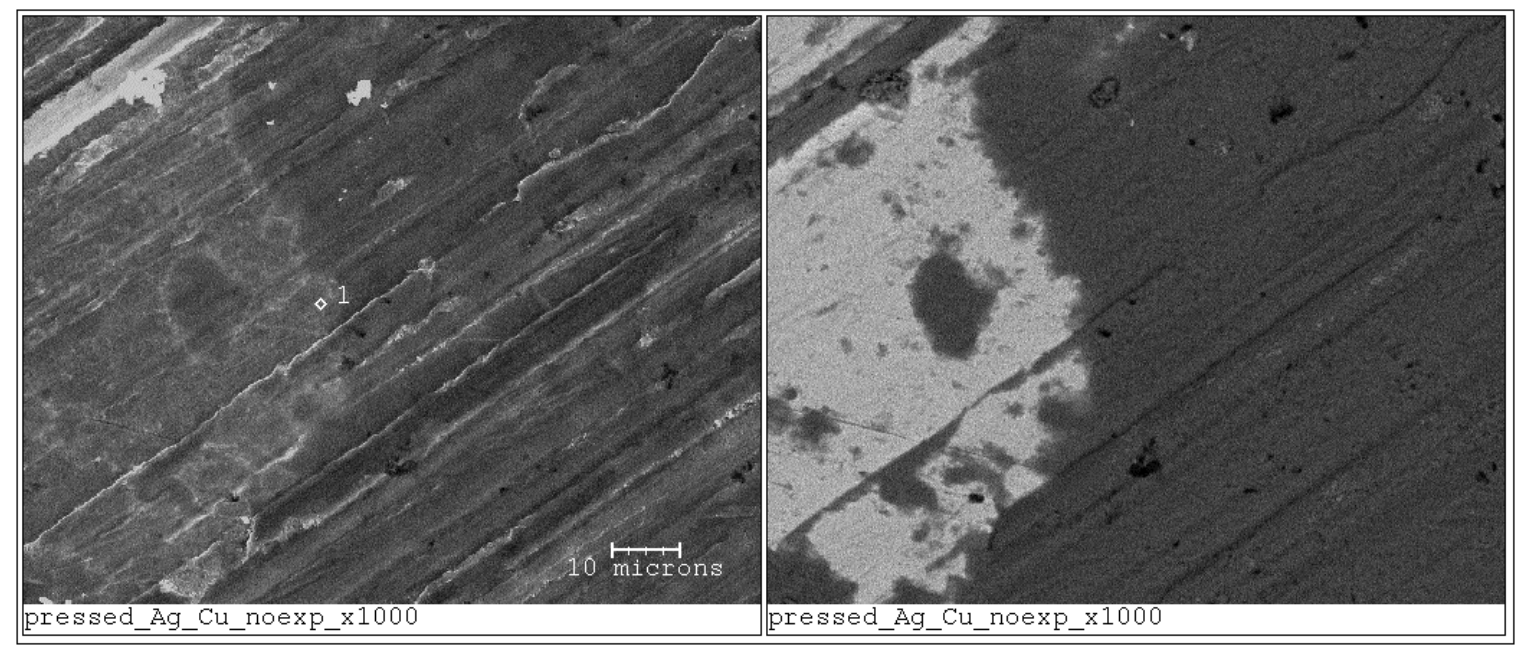

Figure 49: micrograph and backscatter of pressed $\mathrm{Ag} / \mathrm{CuO}$ sample surface
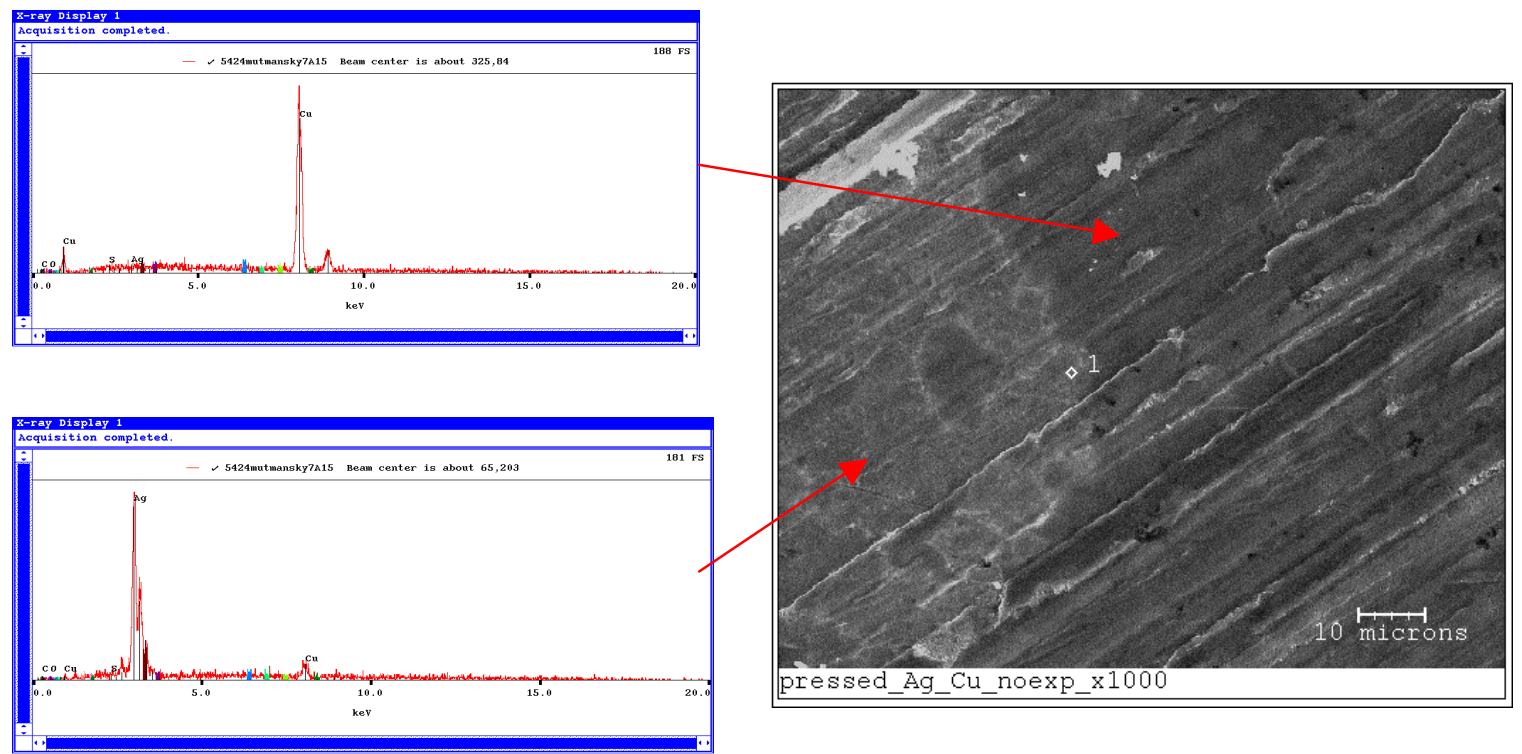

Figure 50: SEM micrograph and $\mathrm{x}$-ray spectrum of pressed $\mathrm{Ag} / \mathrm{CuO}$ sample 
The $\mathrm{x}$-ray spectrum verifies that the area to the right hand edge of the sample is copper-oxide, while the area to the left is silver. The copper-oxide initially appears as a smooth layer, however, it is actually made up of very small particles $(30-50 \mathrm{~nm})$, much like those seen at the surface of the unexposed sterling silver sample.

These samples were exposed to the same high-temperature conditions as the sterling silver samples. Every two weeks the pressed $\mathrm{CuO} / \mathrm{Ag}$ samples were removed from the furnace so that the interface between the silver and copper-oxide could be examined. The SEM backscatter images shown below illustrate the changing surface of the sample during exposure. 


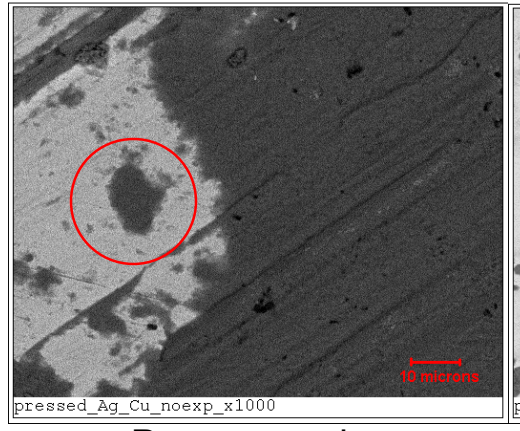

Pre-exposed

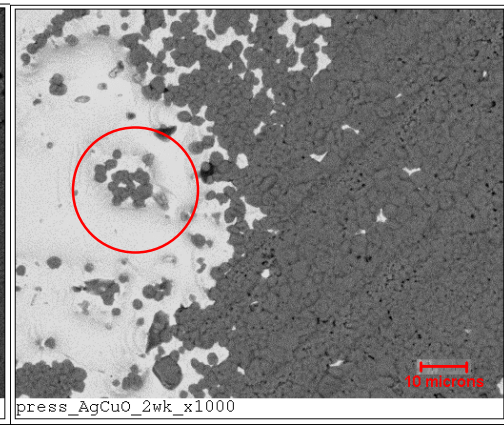

2-weeks
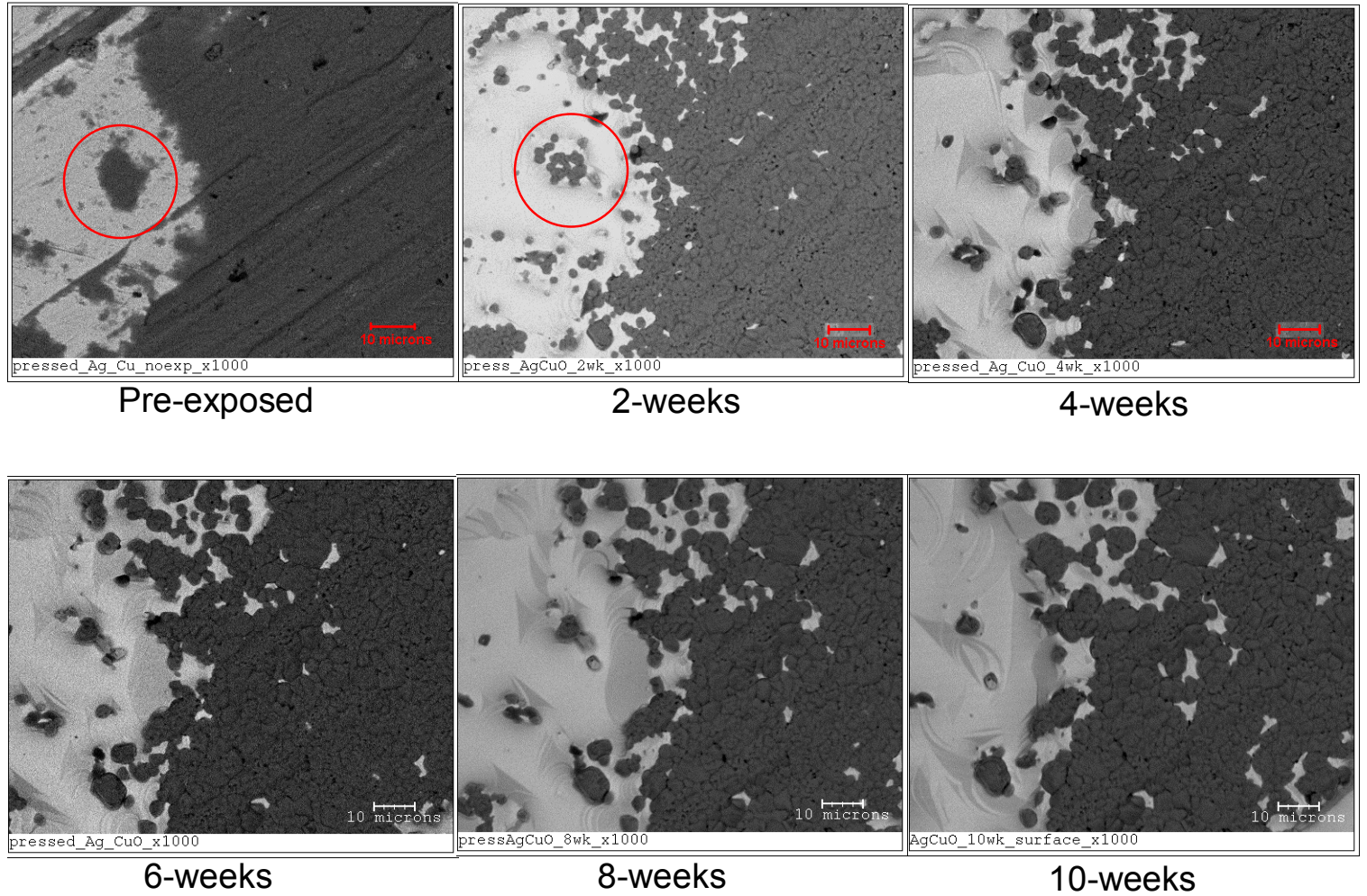

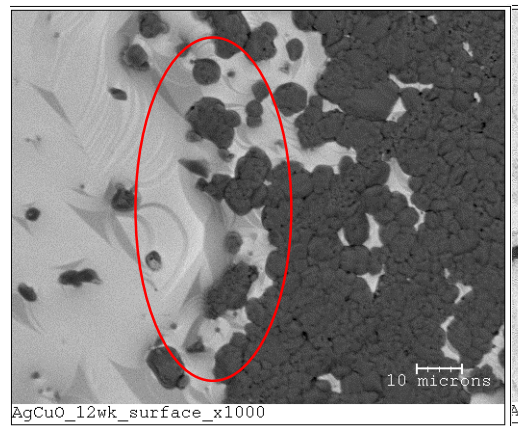

12-weeks

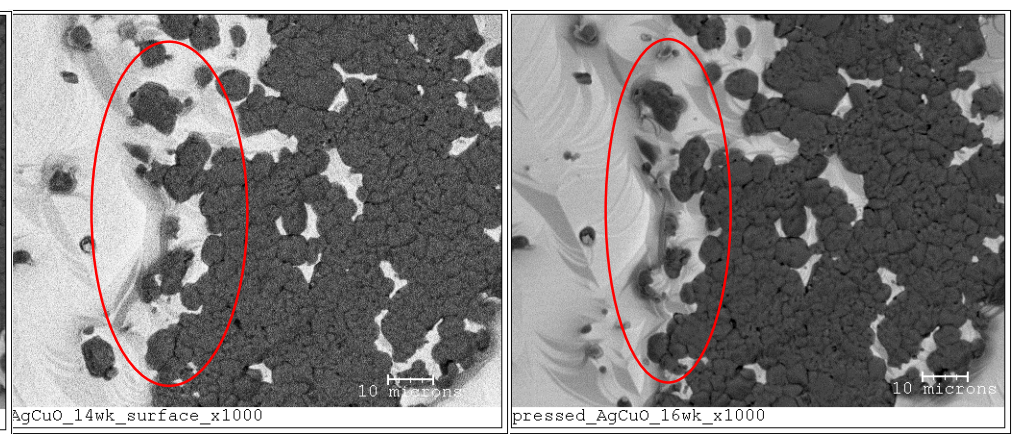

14-weeks

16-weeks

Figure 51: Time-series, pressed $\mathrm{Ag} / \mathrm{CuO}$ sample, low-magnification

It does appear from the backscatter image that silver is more volatile when in close proximity with copper-oxide particles. It appears as though the "island" (circled in red) on the left side of the image is beginning to sink into the silver as recession occurs around it, until it finally disappears by week 16. It also appears that the silver at the edge of the copper-oxide region (also circled in red) is undergoing more dramatic surface 
changes by weeks 16 , showing that silver is less stable in regions of copper-oxide particles. This may indicate that silver is indeed lost more rapidly at the interface between silver and copper-oxide, and shows how the addition of copper-oxide to pure silver could actually be detrimental to the performance of the silver/copper-oxide cermet operating in high-temperature conditions.

In order to more closely examine the interface behavior between silver and copper-oxide, higher magnification imaging of the sample was also carried out. The series of images illustrate the backscatter surface of the sample, the SEM micrographs along with the backscatter are shown in the appendix in larger format. 


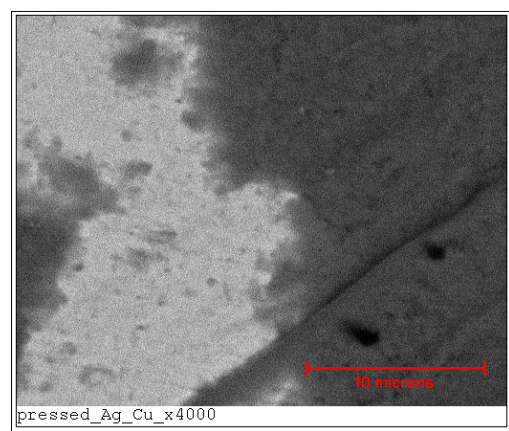

Pre-exposed

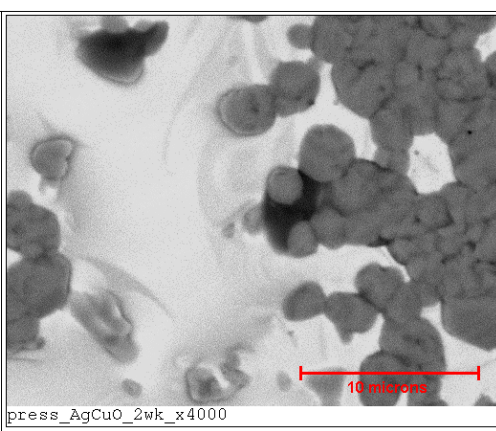

2-weeks

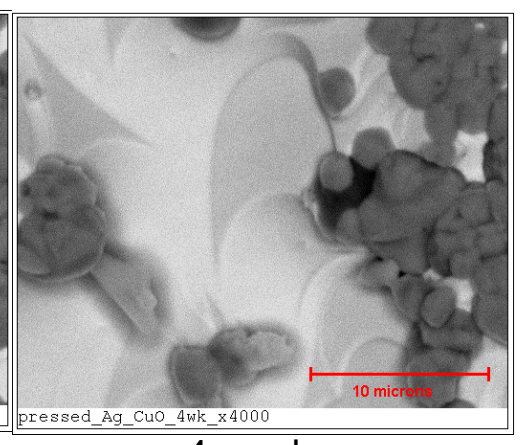

4-weeks
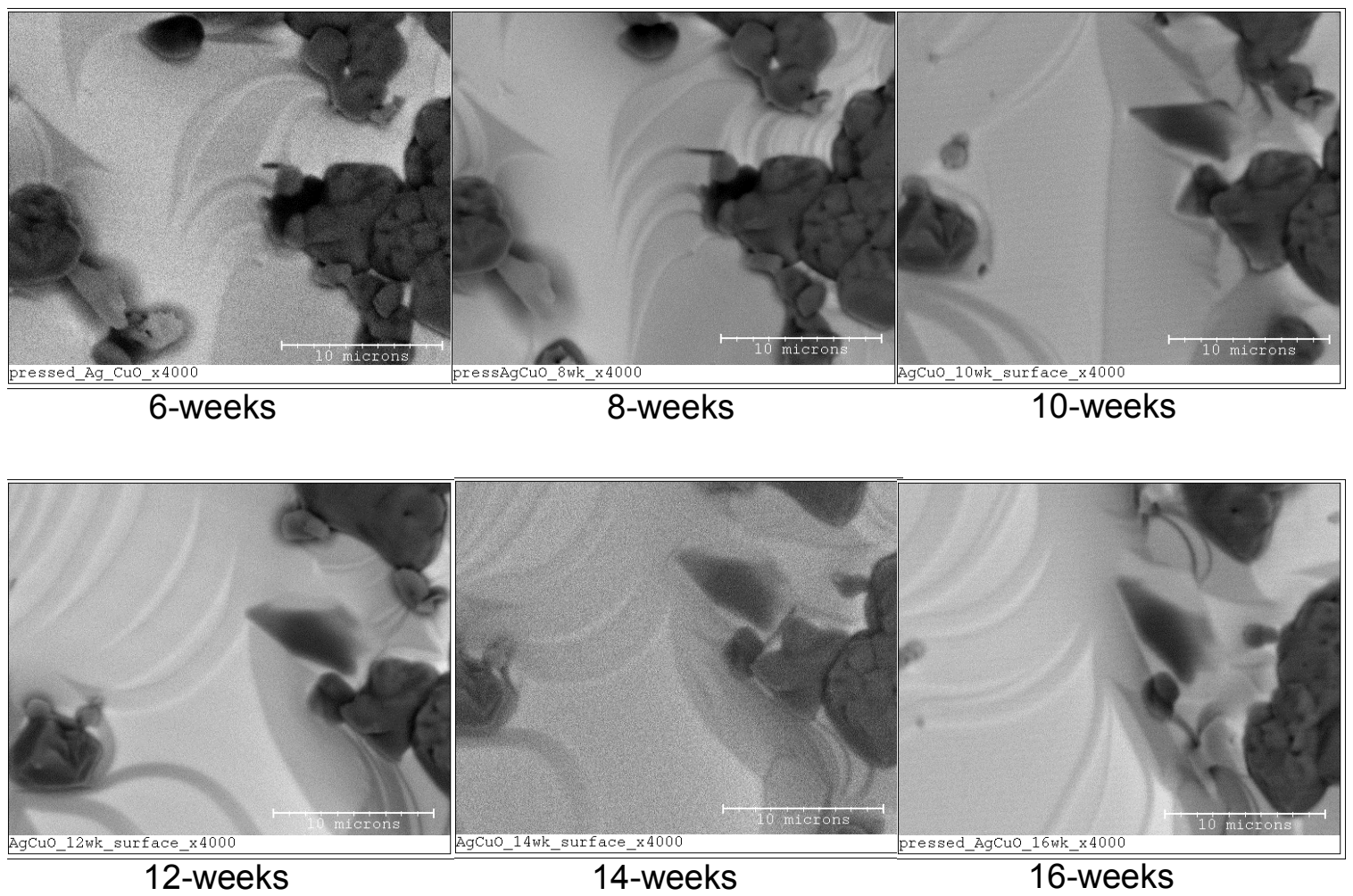

Figure 52: Time-series, pressed Ag/CuO sample, low-magnification

Copper-oxide particles can be seen to coarsen after two weeks of hightemperature exposure. Faceting is also developing on the sample surface.

As the high-temperature exposure proceeds, continued coarsening of the copperoxide particles is observed as well as continued faceting of the silver. It appears that faceting is becoming relatively pronounced between the copper-oxide particles. 
At week ten it appears that microstructure changes have taken place on the righthand side of the sample, possible exposing copper-oxide previously beneath the silver surface. Changes in faceting from previous weeks are also evident. These images illustrate a potential problem with copper-oxide with regards to stability.

\subsubsection{Ag/LSM Sample}

Due to the volatility of copper-oxide in terms of migration, agglomeration and coarsening of the particles, silver/LSM samples were fabricated by the same means as the silver/copper-oxide samples previously mentioned in order to examine the effects of high-temperature exposure on LSM particles as well as the interaction between silver and LSM. The following figure illustrates an SEM micrograph of the surface of the sample with x-ray spectrums to verify the location of LSM particles.
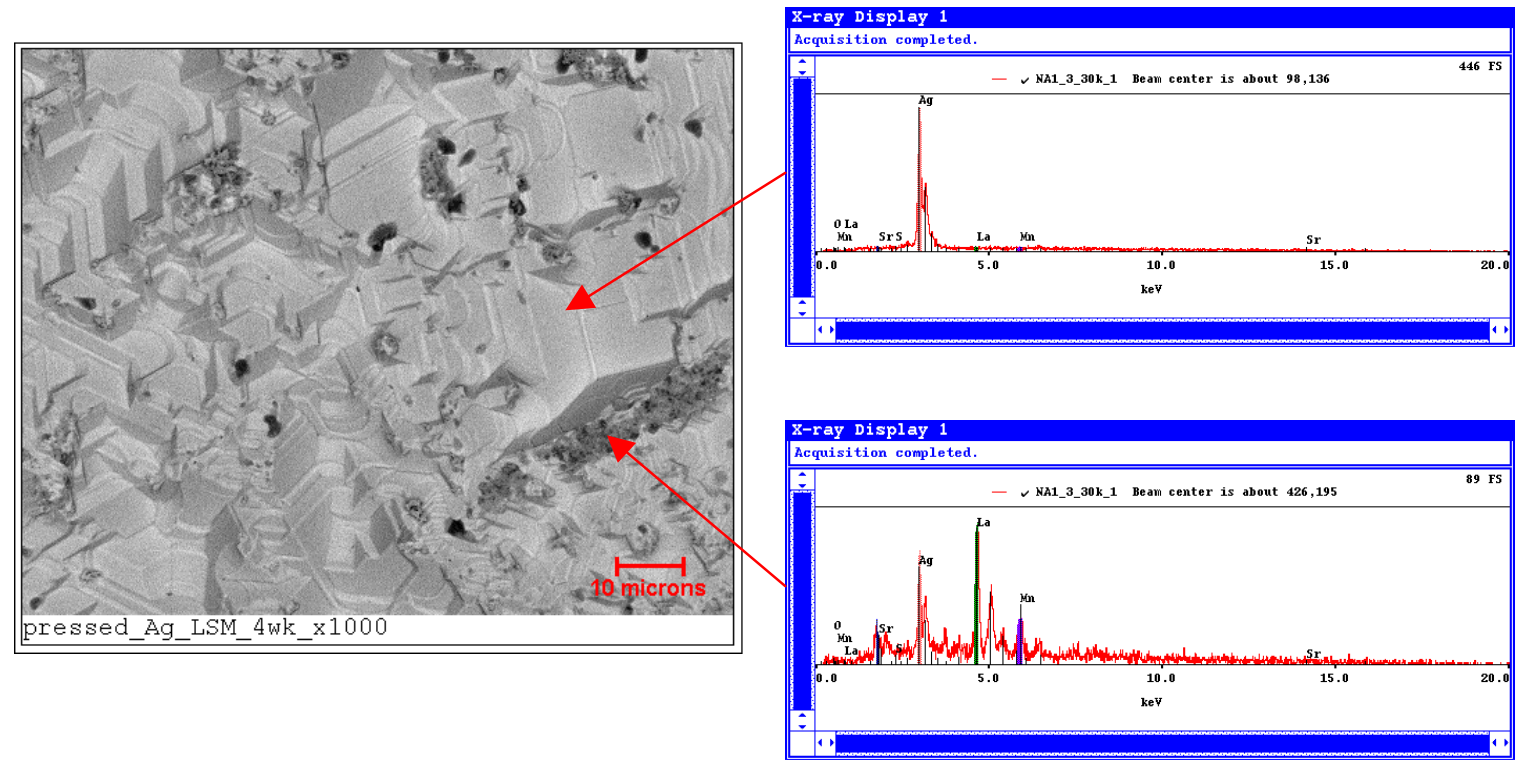

Figure 53: Ag/LSM sample surface with x-ray spectrums 
The same method of time-series analysis used on the silver/copper-oxide samples was used to examine these samples. A low-magnification time series of the silver/LSM sample is shown in the following figure.
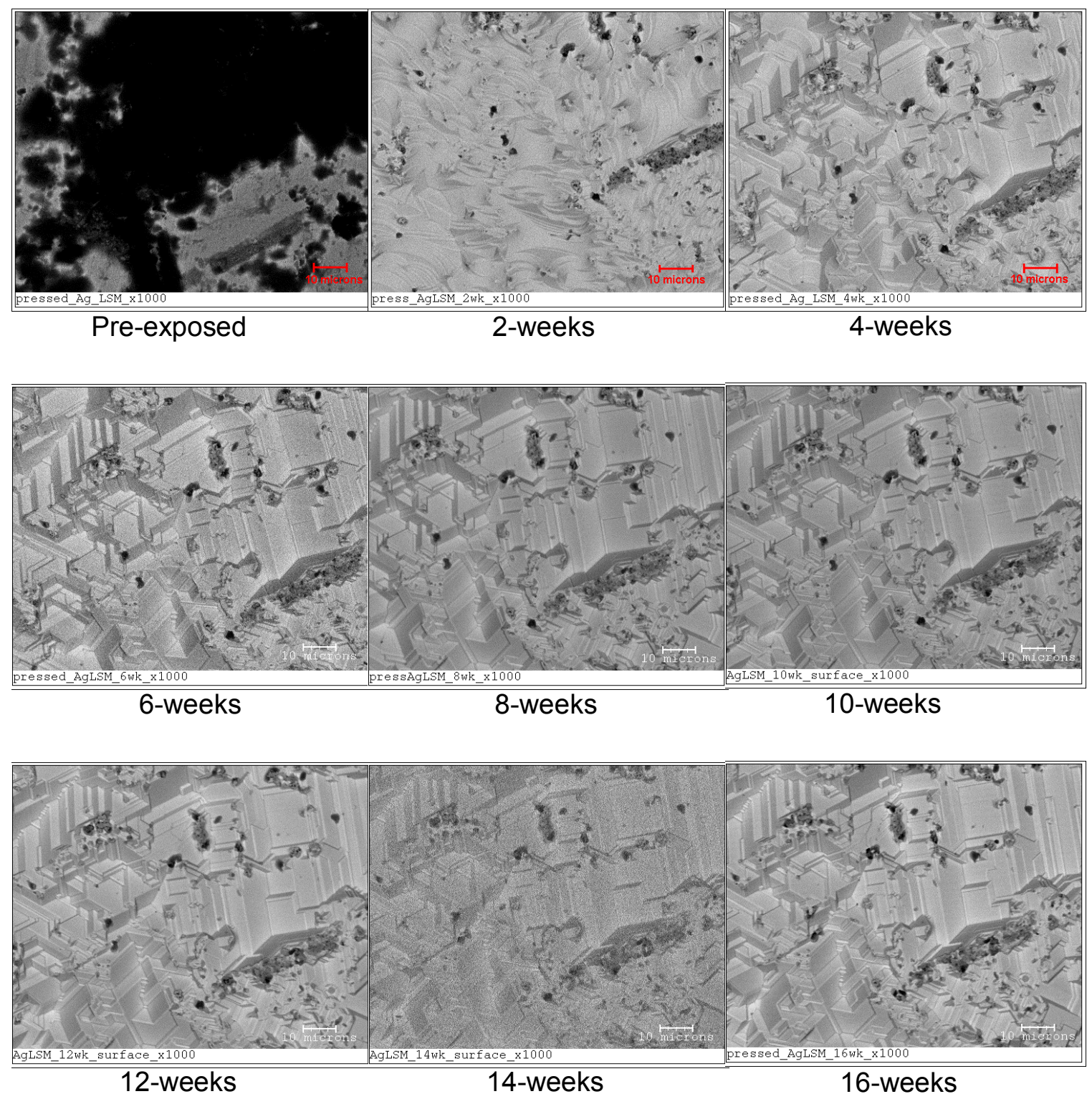

Figure 54: Time-series, pressed Ag/LSM sample, low-magnification 
The LSM particles indicated in the sample appear relatively stable in terms of migration and coarsening throughout the duration of the experiment. The silver surrounding the particles can be seen to proceed through differing stages of faceting, reaching the "stepped" orientation by week 16.

In order to better examine the interaction between silver and LSM at the surface of the sample a higher-magnification SEM time series was also performed. This imaging focused on an area exhibiting more abundant LSM to examine its stability throughout the experiment. 


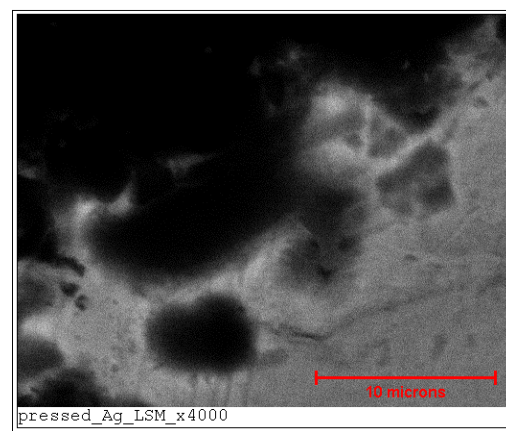

Pre-exposed

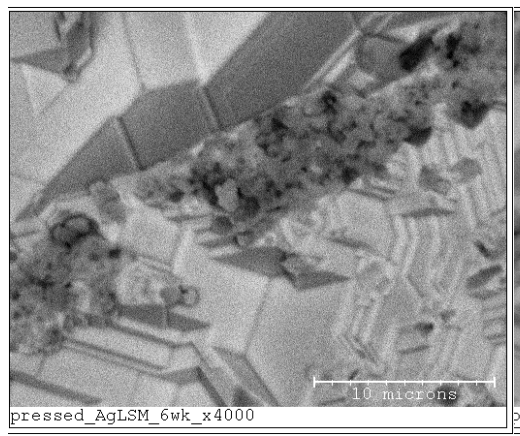

6-weeks

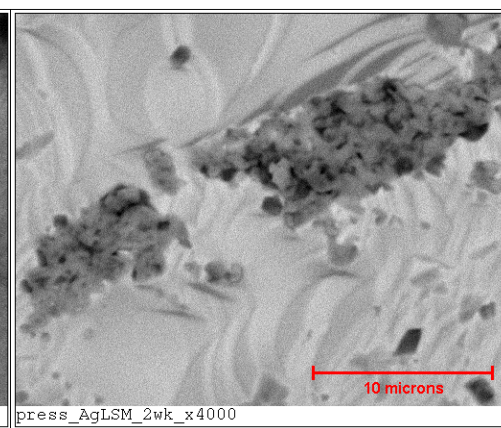

2-weeks

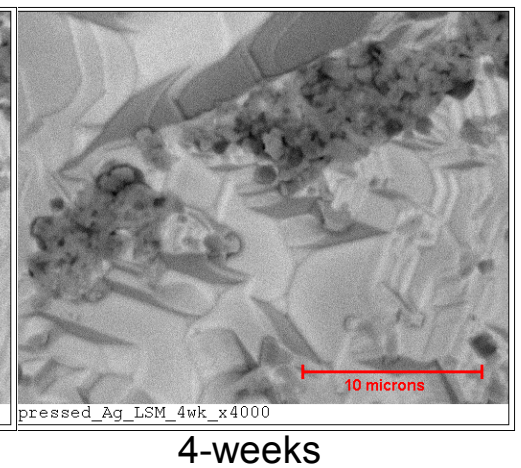

4-weeks
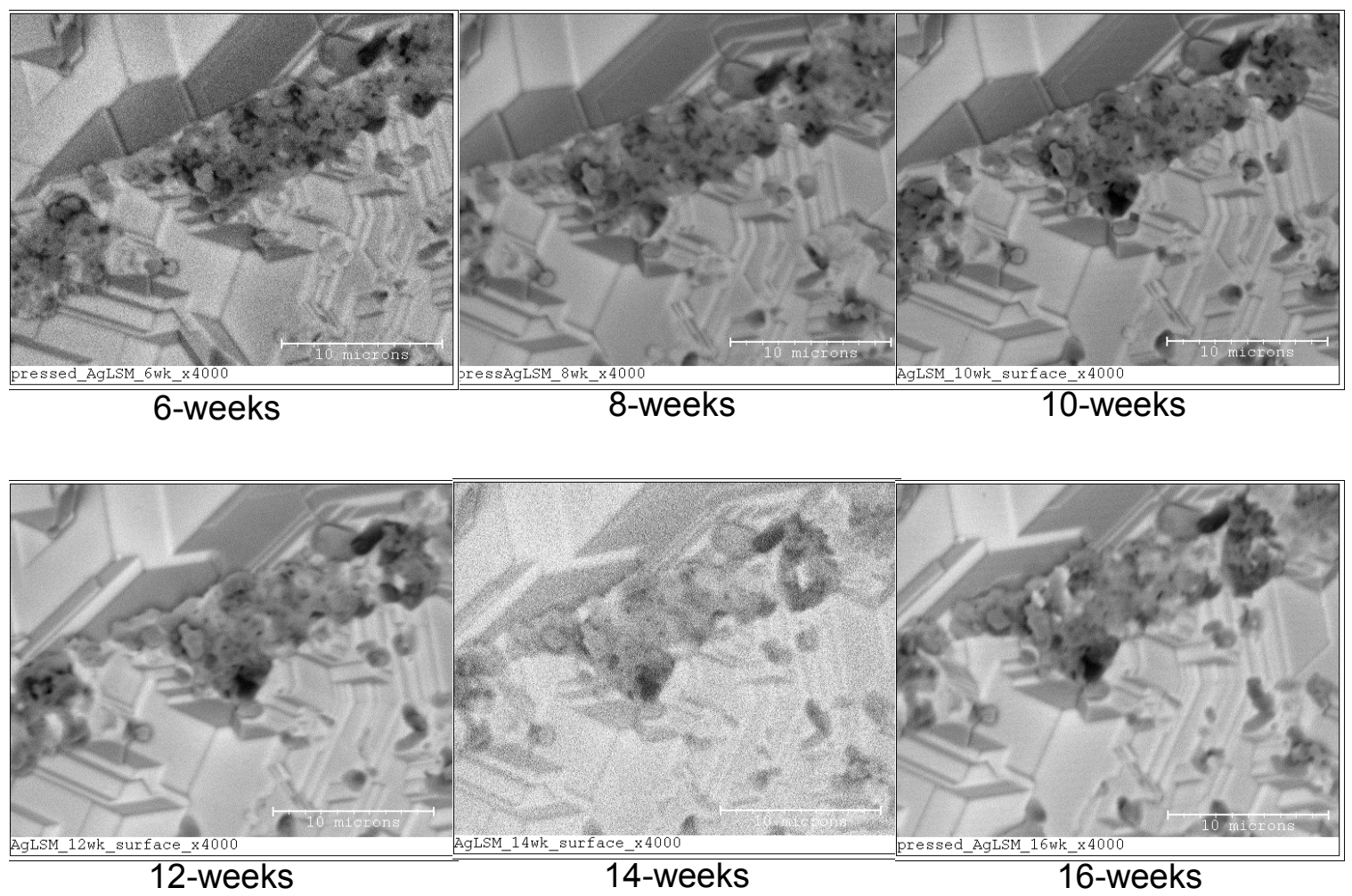

Figure 55: Time-series, pressed Ag/LSM sample, high-magnification

The LSM particles examined appear to be very stable throughout the entire duration of high-temperature exposure. The particles do not appear to coarsen or agglomerate, even after four months. It also appears that the presence of the copperoxide does not affect the faceting of the silver surrounding it, allowing it to reach the "stepped" orientation like that seen in the pure silver samples. It appears that LSM may 
be a more stable alternative to copper-oxide as a protective oxide if a suitable composite material can be fabricated.

\subsection{COMPARISON OF FACETING}

As mentioned, it is believed based on the data recorded as well as the SEM analysis of various samples that the differences in evaporation rate are related to the differing surface orientations observed throughout the high-temperature exposure. It is believed that the pure silver samples are able to reach an orientation that is less-favorable to evaporation, while the copper-oxide particles in the sterling silver act to inhibit faceting of the silver surface, not allowing the sample to reach the desired lowevaporation surface orientation. The following images compare the faceting of a sterling silver sample and a pure silver sample after two and seven weeks of exposure respectively.
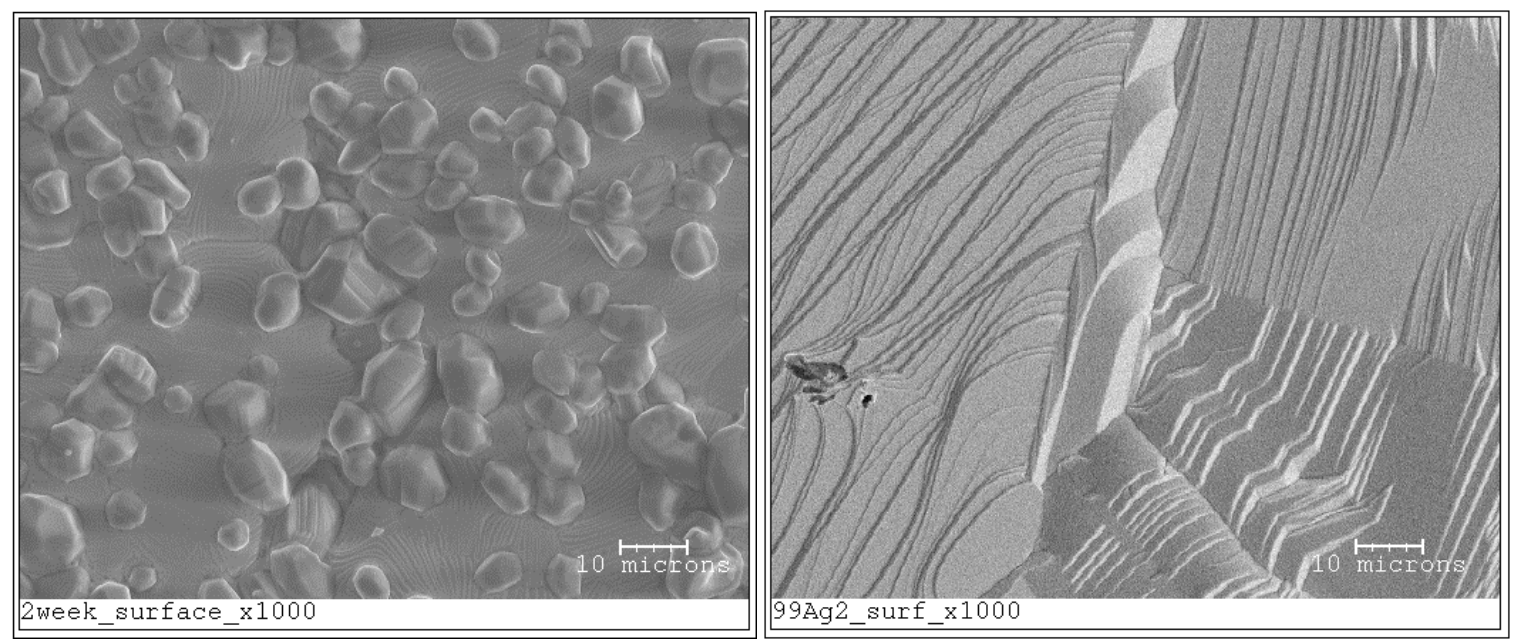

Figure 56: Comparison of faceting of sterling silver sample and pure silver sample (2-week exposure) 

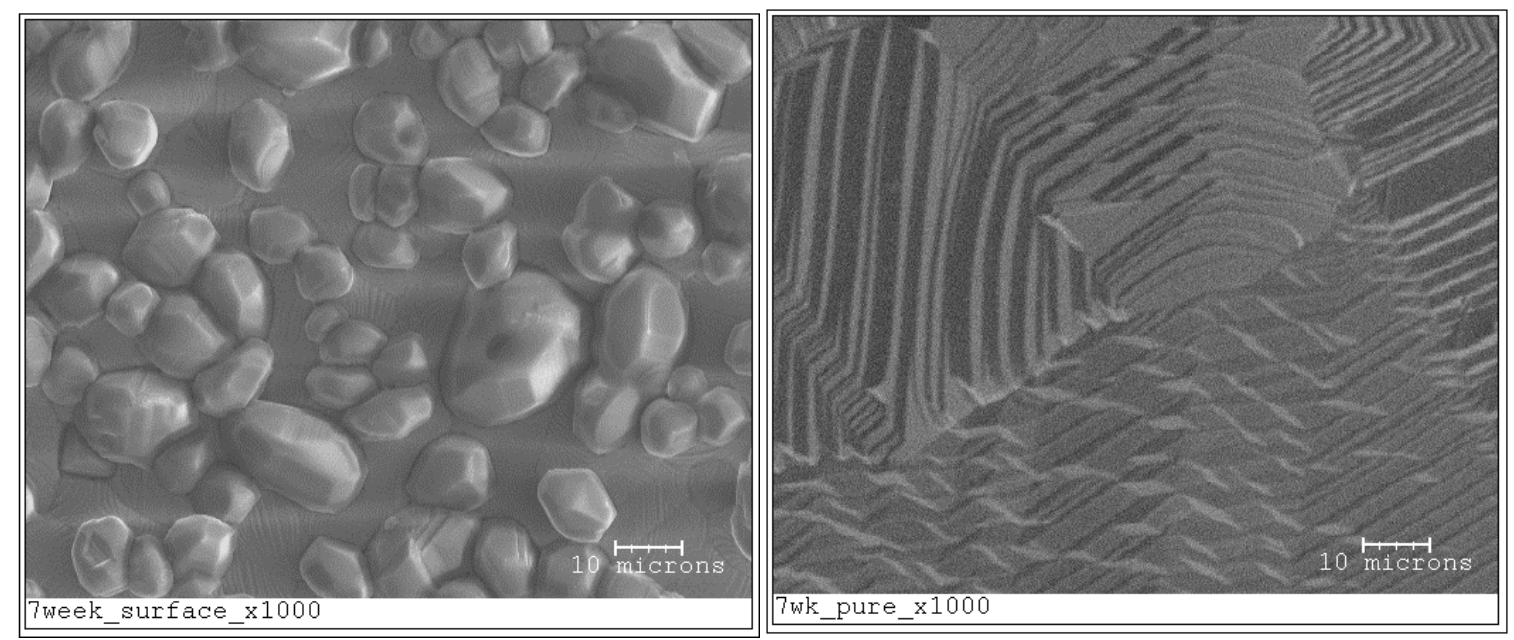

Figure 57: Comparison of faceting of sterling silver sample and pure silver sample (7-week exposure)

Faceting is evident in the sterling samples which can be seen as small striations at the surface, however the faceting is clearly not as pronounced as in the pure silver sample in the image on the right. It may be possible that the orientation of the pure silver sample is less conducive to evaporation than that of the silver on the left, therefore allowing the sterling silver sample to maintain a higher rate of mass loss than the pure silver. 
CHAPTER 6: Discussion and Conclusion

\subsection{DISCUSSION AND CONCLUSION}

The original intention of this research was to show that the addition of copperoxide to silver would be beneficial in inhibiting the loss of silver during high-temperature exposure. The data appears to suggest that in this particular case the opposite is true. It appears that the thickness reduction of the pure samples decreases over time. Meanwhile, the thickness reduction of the sterling sample remains relatively constant over time, at a rate twice that of pure silver.

This appears to be due to instability in the copper-oxide that may cause an increase in silver evaporation at the interface between the silver and copper-oxide particles as can be seen in SEM micrographs and is further evident upon comparison of the evaporation rate of the sterling silver samples and the pure silver samples. This could be due to the surface orientation of the silver in each sample. It can be seen in the sterling silver samples that the silver surrounding the copper-oxide particles does not exhibit as dramatic faceting as that seen on the surface of the pure silver samples. What could be happening is the pure silver samples are reaching a surface orientation that is not favorable to evaporation, while the copper-oxide particles at the surface of the sterling silver samples do not allow the silver to ever reach this same orientation. Microscopy of pure silver after one week of high-temperature exposure shows the least pronounced faceting in the experiment and is also the week the sample experienced its highest rate of evaporation. Evaporation in the pure silver decreased as faceting increased as seen from the collected data and SEM imaging, indicating there may be a relation between the two. 
During the first several weeks of exposure, it is likely that surface orientation favorable to evaporation are still present, allowing this higher rate of silver evaporation. Afterward, the remaining surface orientations are no longer favorable for silver evaporation and the thickness reduction of the samples is seen to stabilize.

This is contrasted by the sterling silver samples where the data shows that after initial oxidation of the sample, the evaporation rate is consistent from weeks to week. Imaging using SEM shows that the surface faceting of the sterling silver is much less pronounced than that of the pure silver samples and shows little change throughout the duration of the experiment, which likely means that the surface orientations favorable to evaporation remain after several weeks of high-temperature exposure. This could be the cause of the sterling silver losing silver faster than the pure silver.

\subsection{CONCLUSION}

The data collected indicates that the sterling silver evaporated roughly twice as fast as its pure silver counterparts. While it was hoped that copper-oxide could act as a suitable protective oxide for the silver cermet, long-term analysis under high-temperature exposure conditions indicates that it may have more detrimental than beneficial attributes.

Analysis of sterling silver samples and samples made by pressing copper-oxide into pure silver indicate that copper-oxide may be too volatile to use with silver under SOFC operating conditions. Copper-oxide particles appeared to coarsen throughout the duration of the experiment and SEM imaging of copper-oxide pressed into silver showed differing behavior of silver in close proximity to copper-oxide particles compared to 
silver at a distance from the particles, indicating that there me be issues regarding copperoxides reactivity with silver under high-temperature conditions.

In contrast, time-series analysis of LSM pressed into silver showed that LSM did not coarsen when exposed to high-temperature conditions and silver in its proximity still exhibited the high degree of faceting seen in the pure silver samples. This indicates that LSM may act as a better protective oxide for SOFC applications and likely should be the focus of future research efforts. 


\section{APPENDIX 1}

SCANNING ELECTRON MICROSCOPY x1000 MAGNIFICATION -- TIME-

SERIES ANAYLSIS OF EARLY STAGE STERLING SILVER SAMPLE
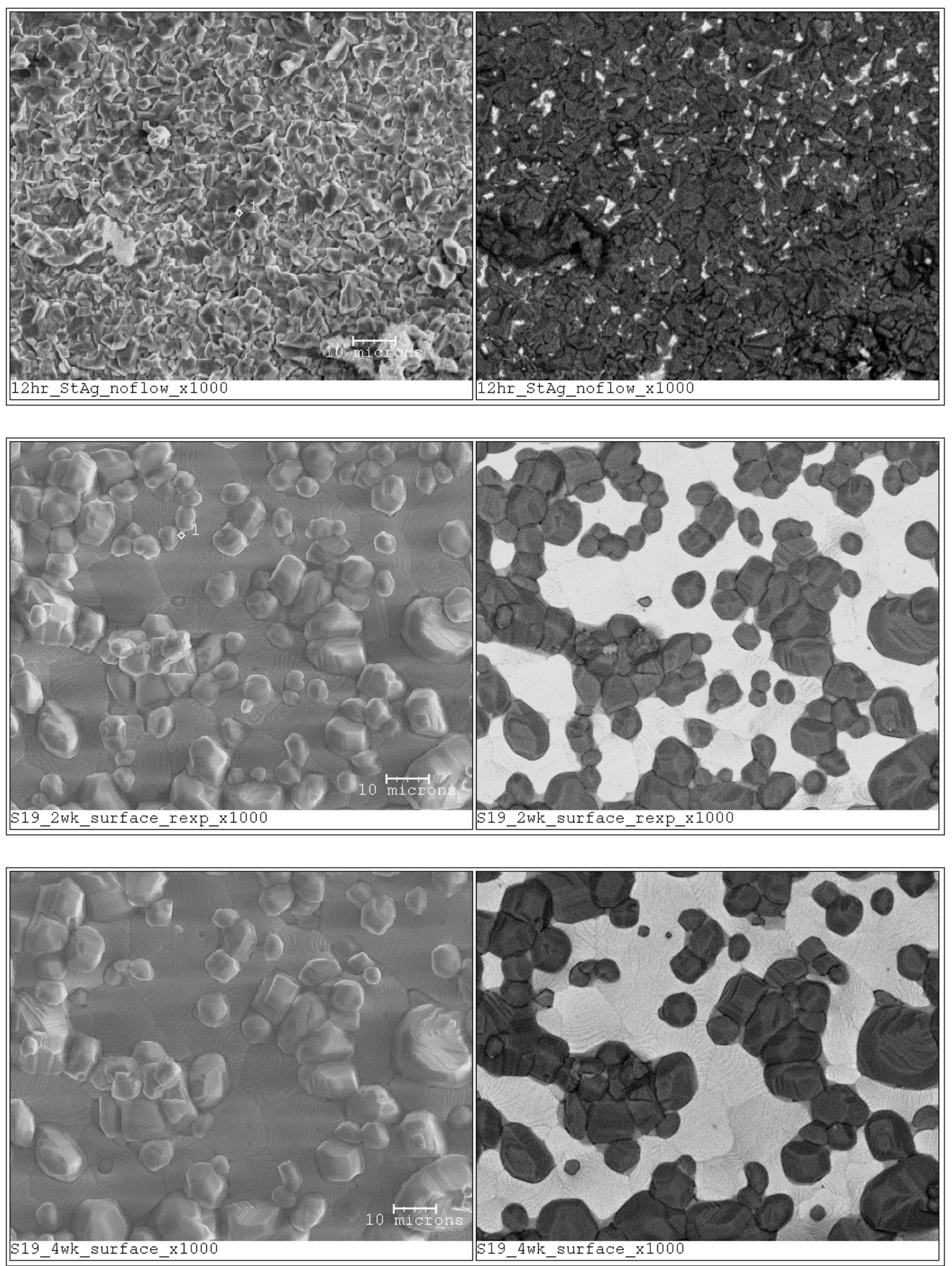

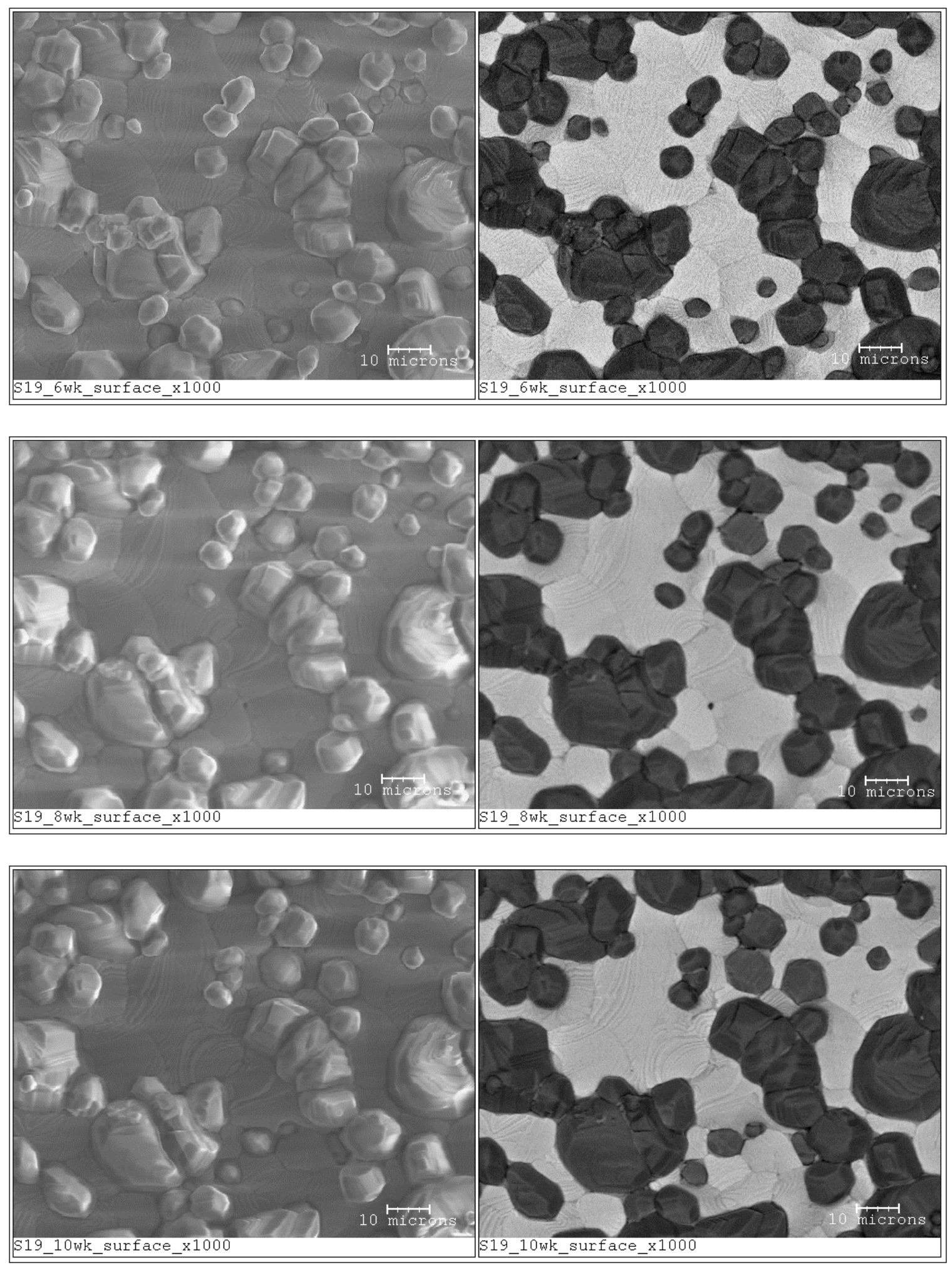

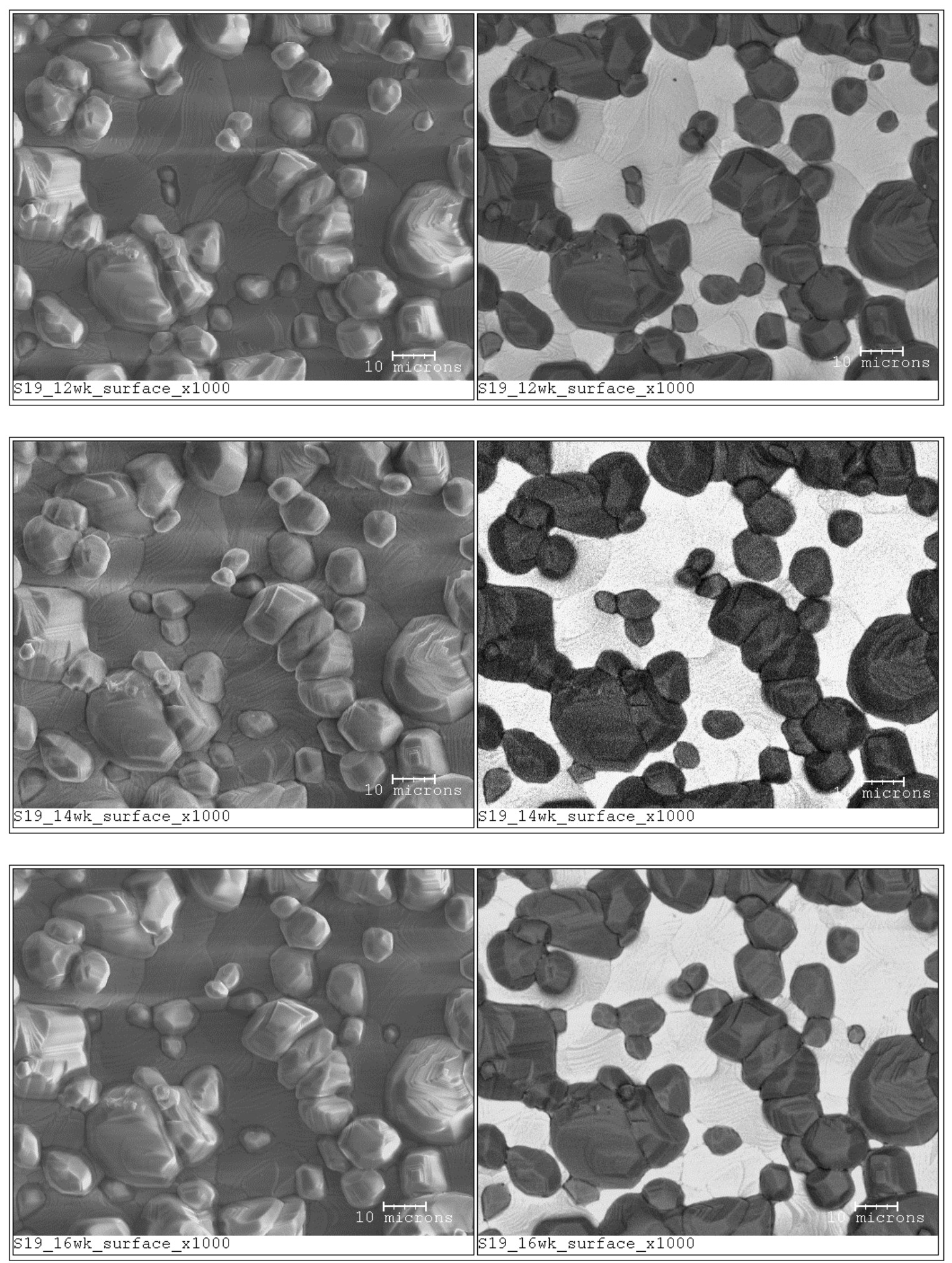
APPENDIX 2

SCANNING ELECTRON MICROSCOPY x4000 MAGNIFICATION -- TIME-

SERIES ANAYLSIS OF EARLY STAGE STERLING SILVER SAMPLE
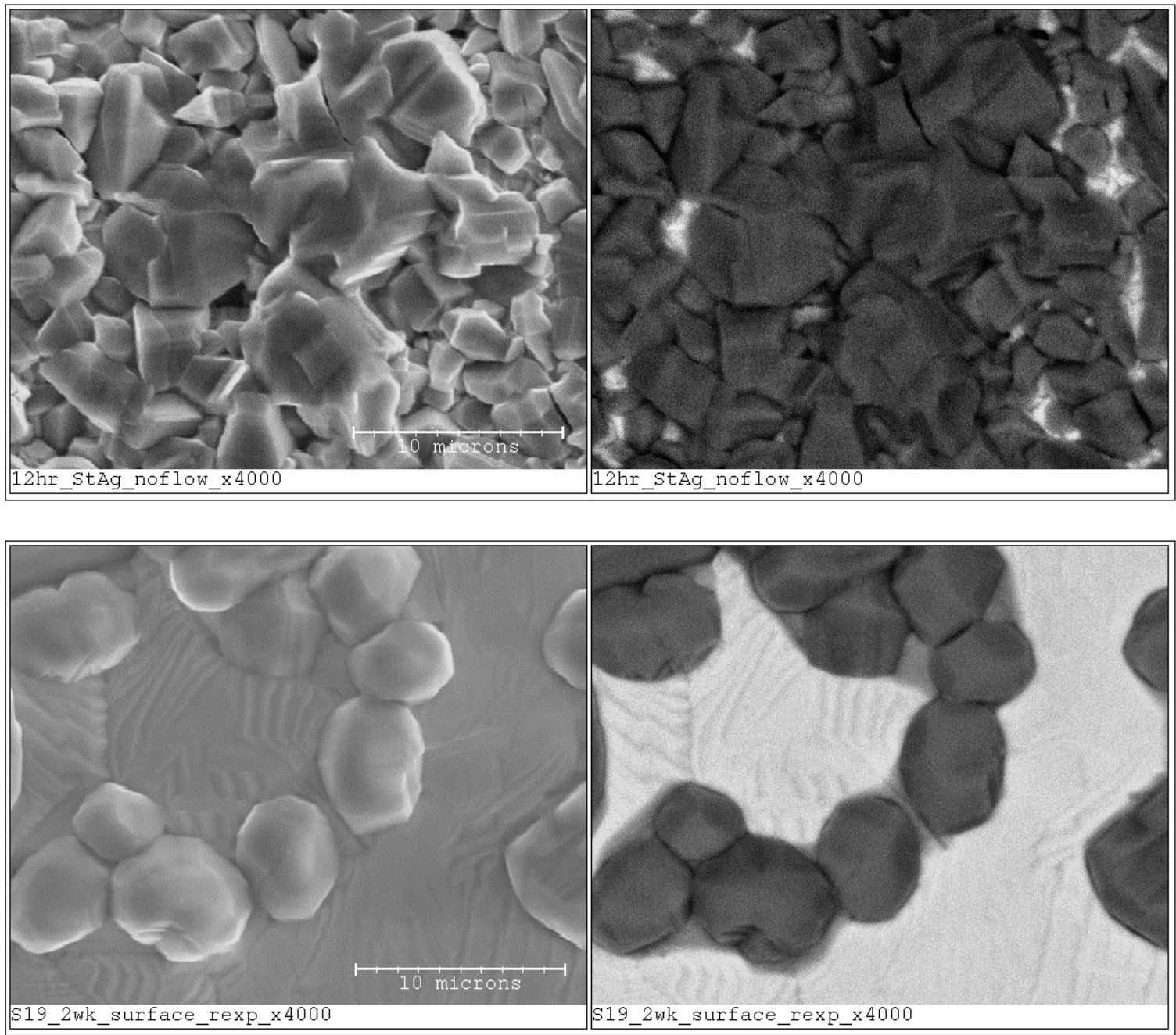

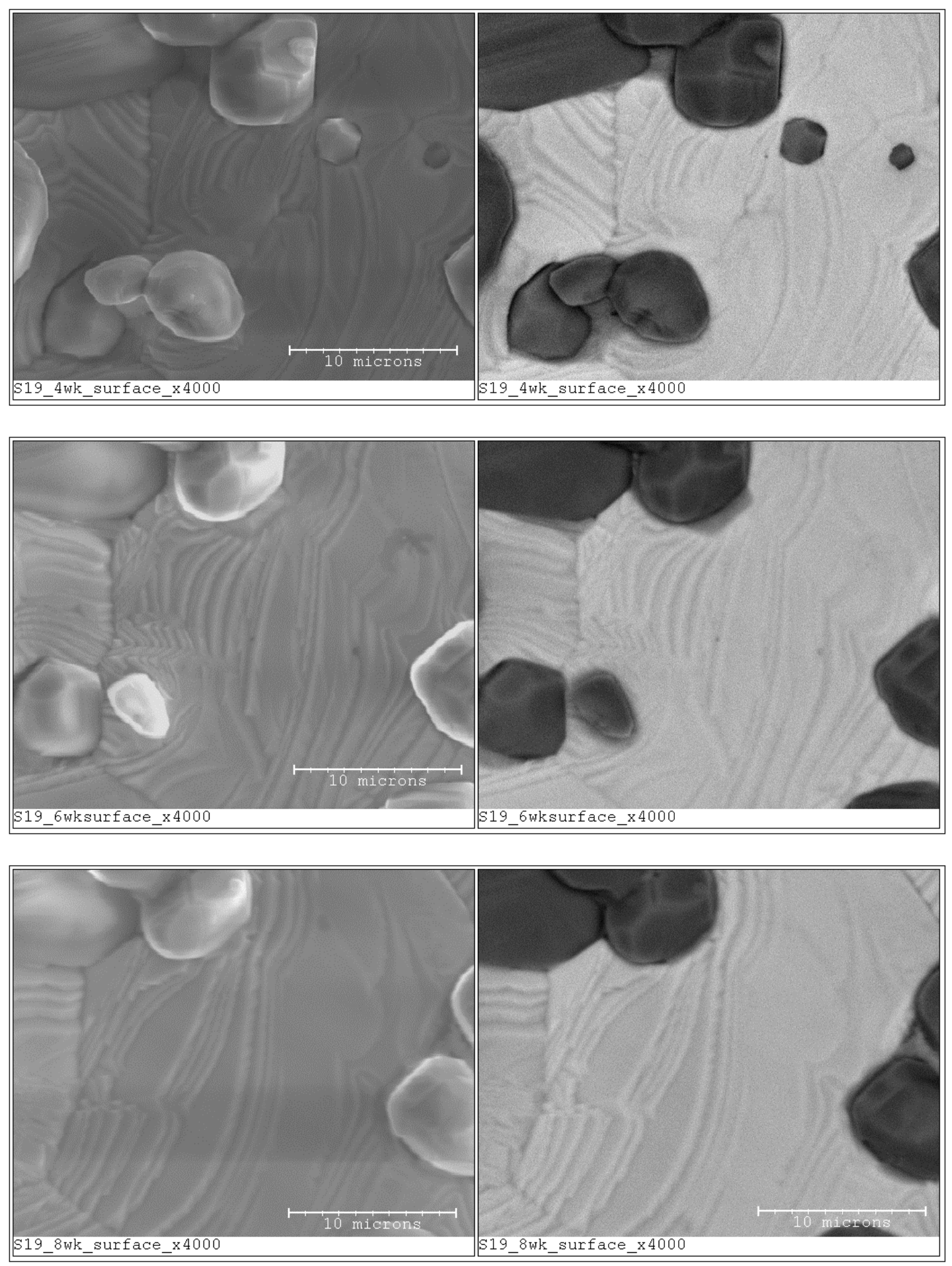

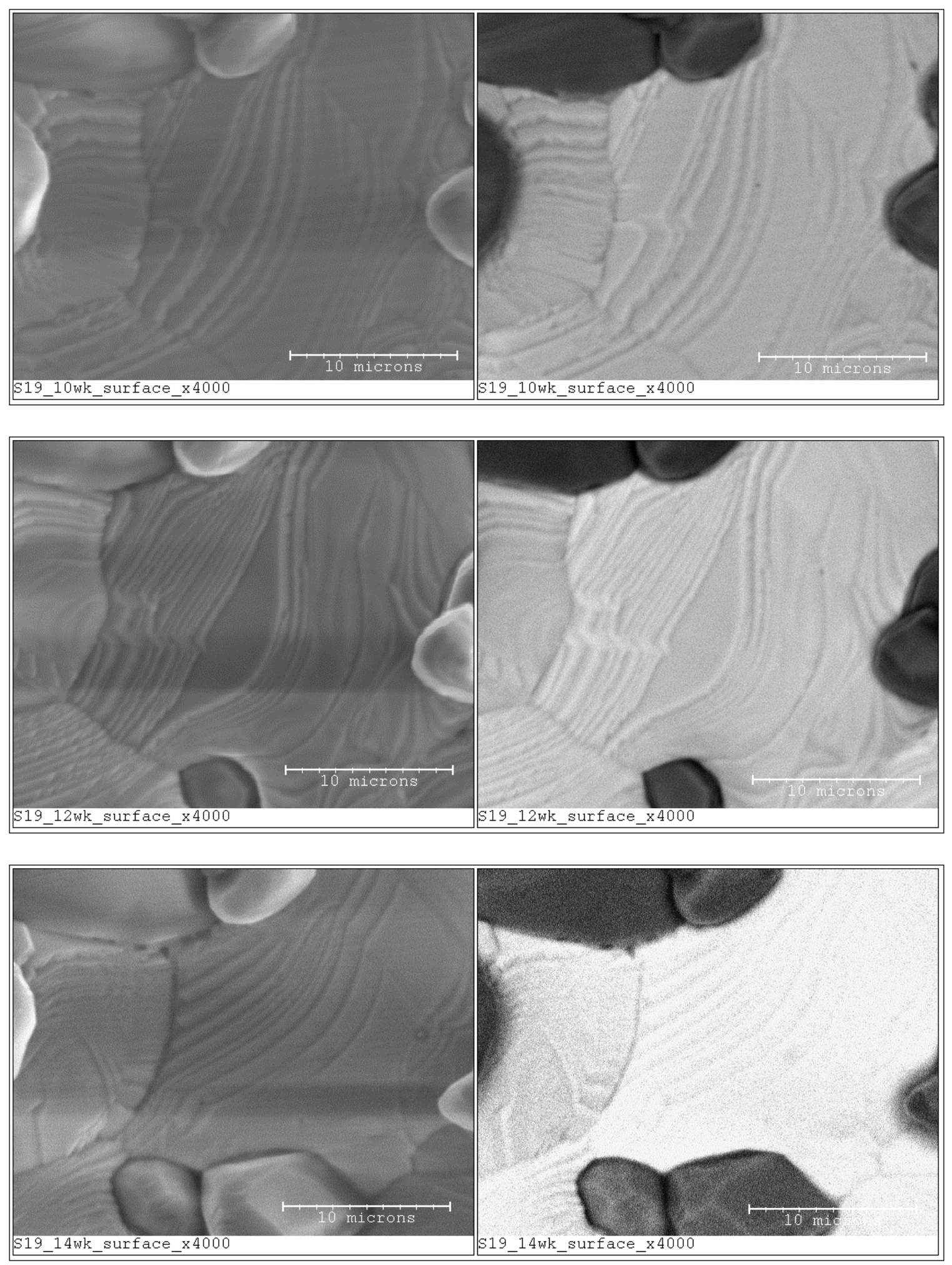


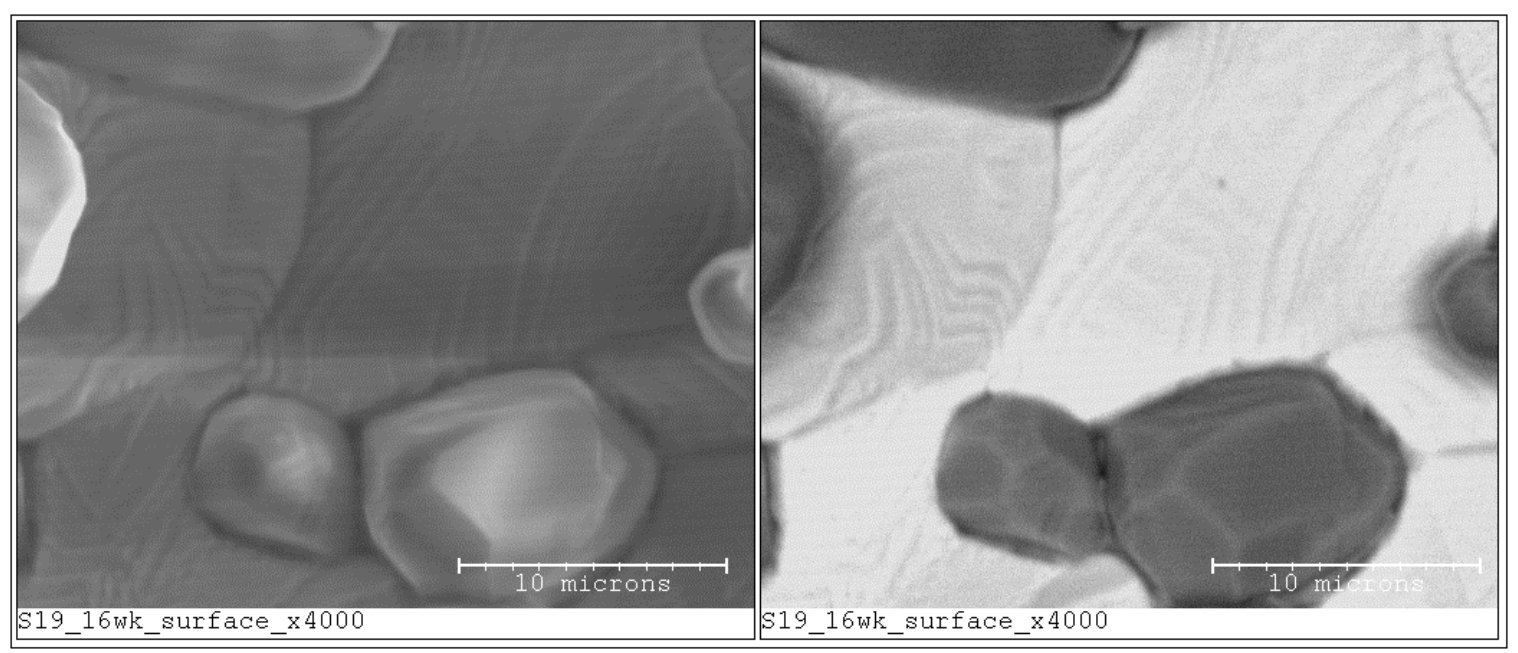


APPENDIX 3

SCANNING ELECTRON MICROSCOPY x1000 MAGNIFICATION -- TIME-

SERIES ANAYLSIS OF LATER STAGE STERLING SILVER SAMPLE
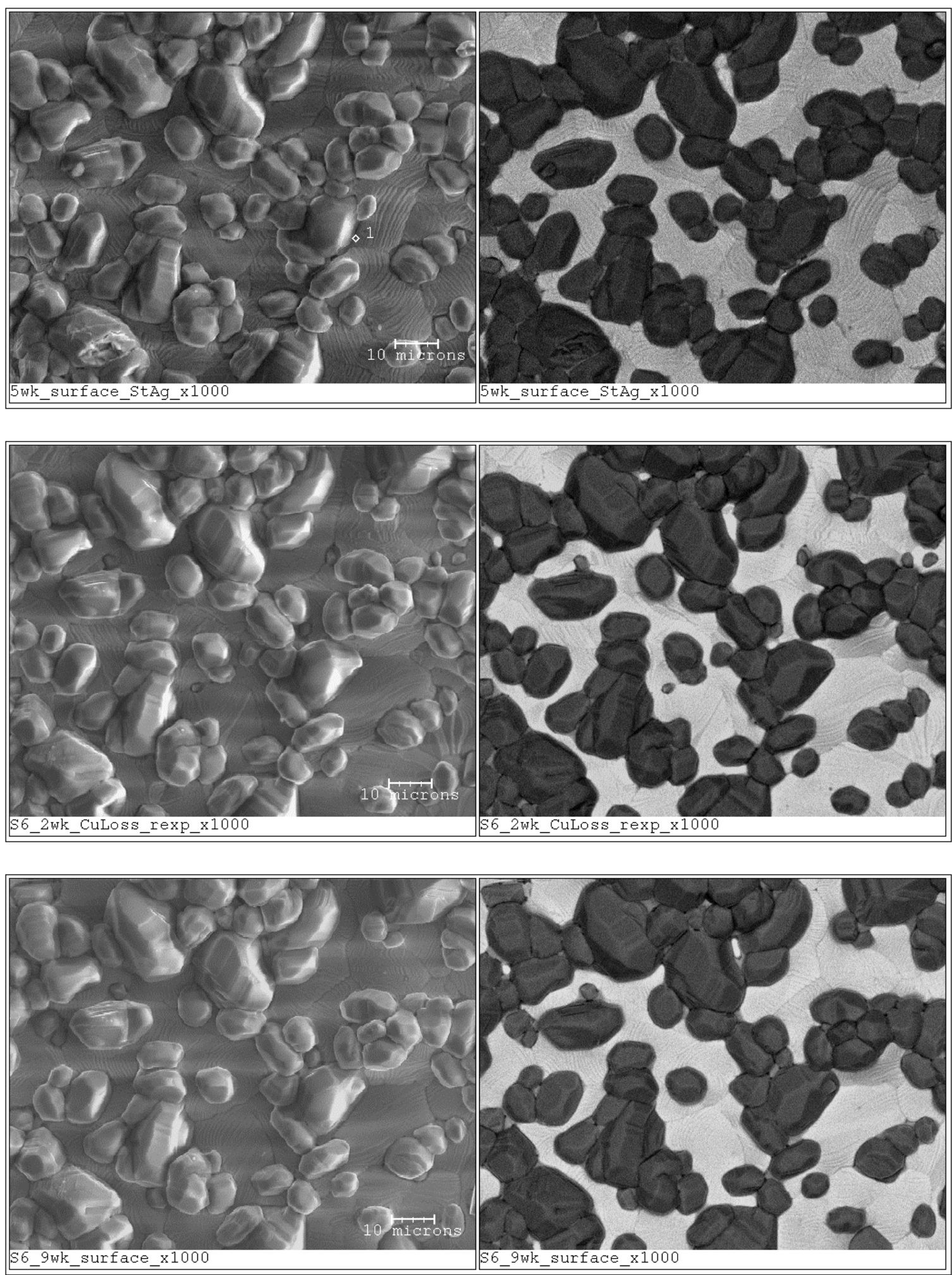

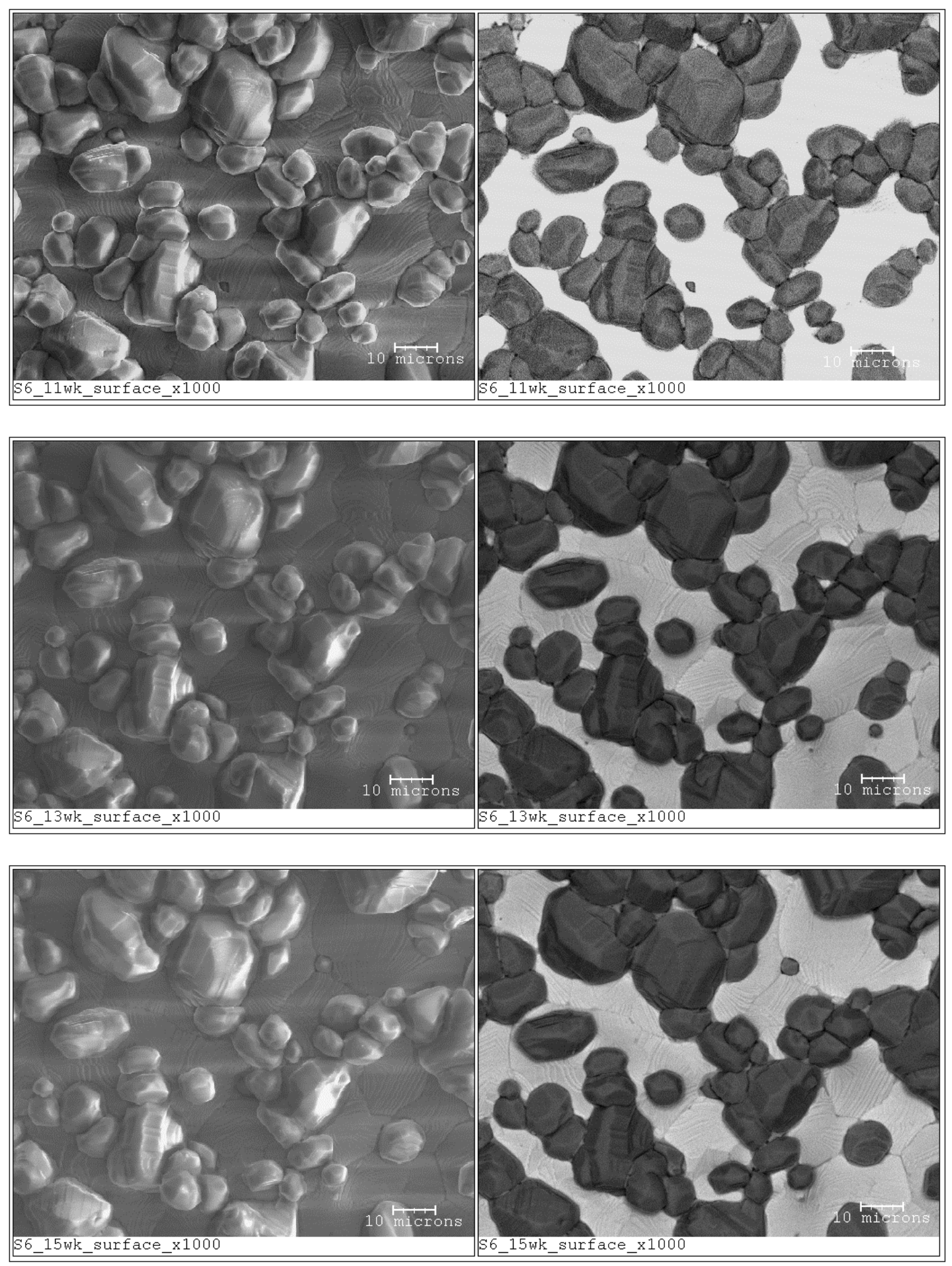

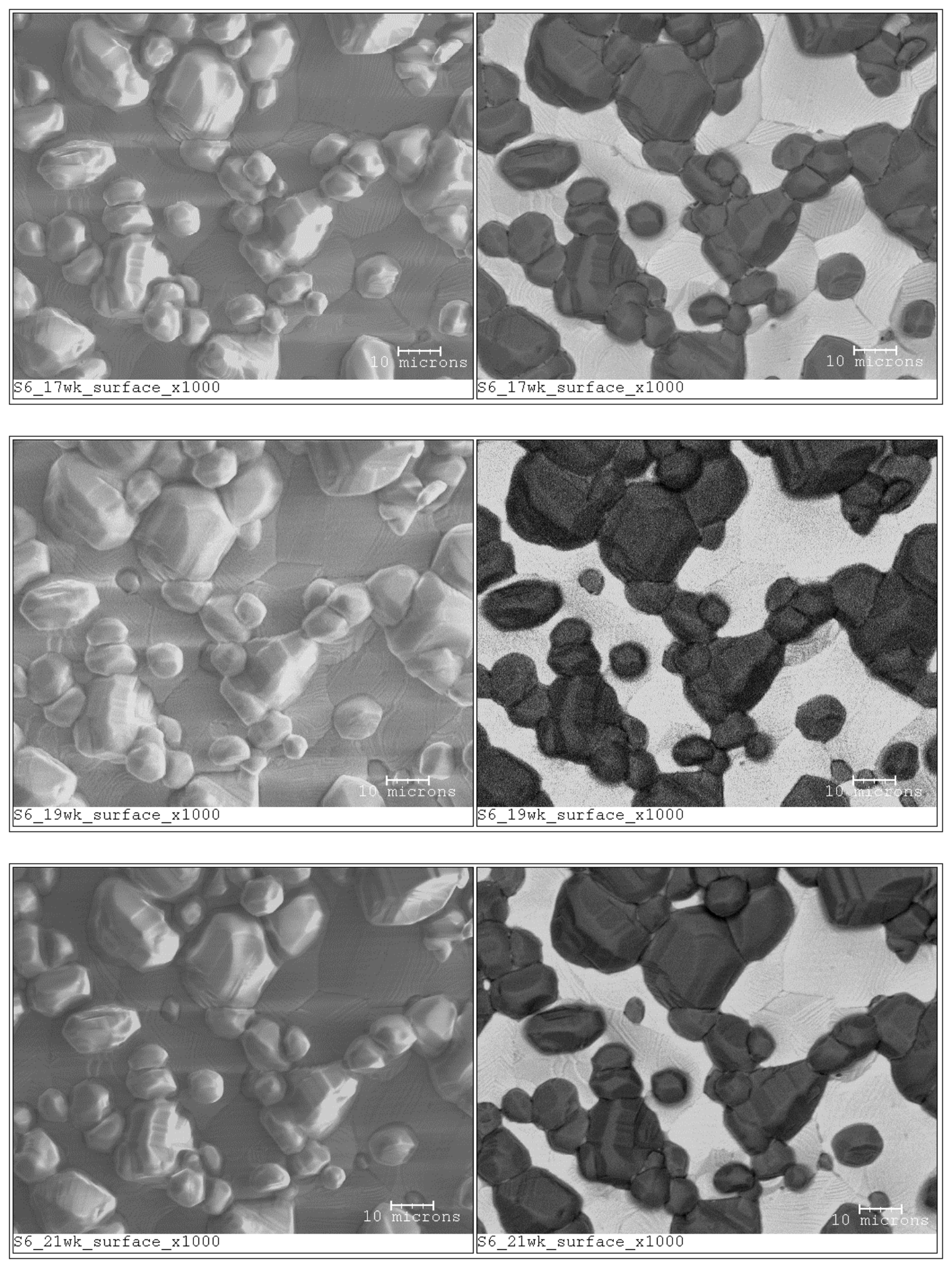
APPENDIX 4

SCANNING ELECTRON MICROSCOPY x4000 MAGNIFICATION -- TIME-

SERIES ANAYLSIS OF LATER STAGE STERLING SILVER SAMPLE
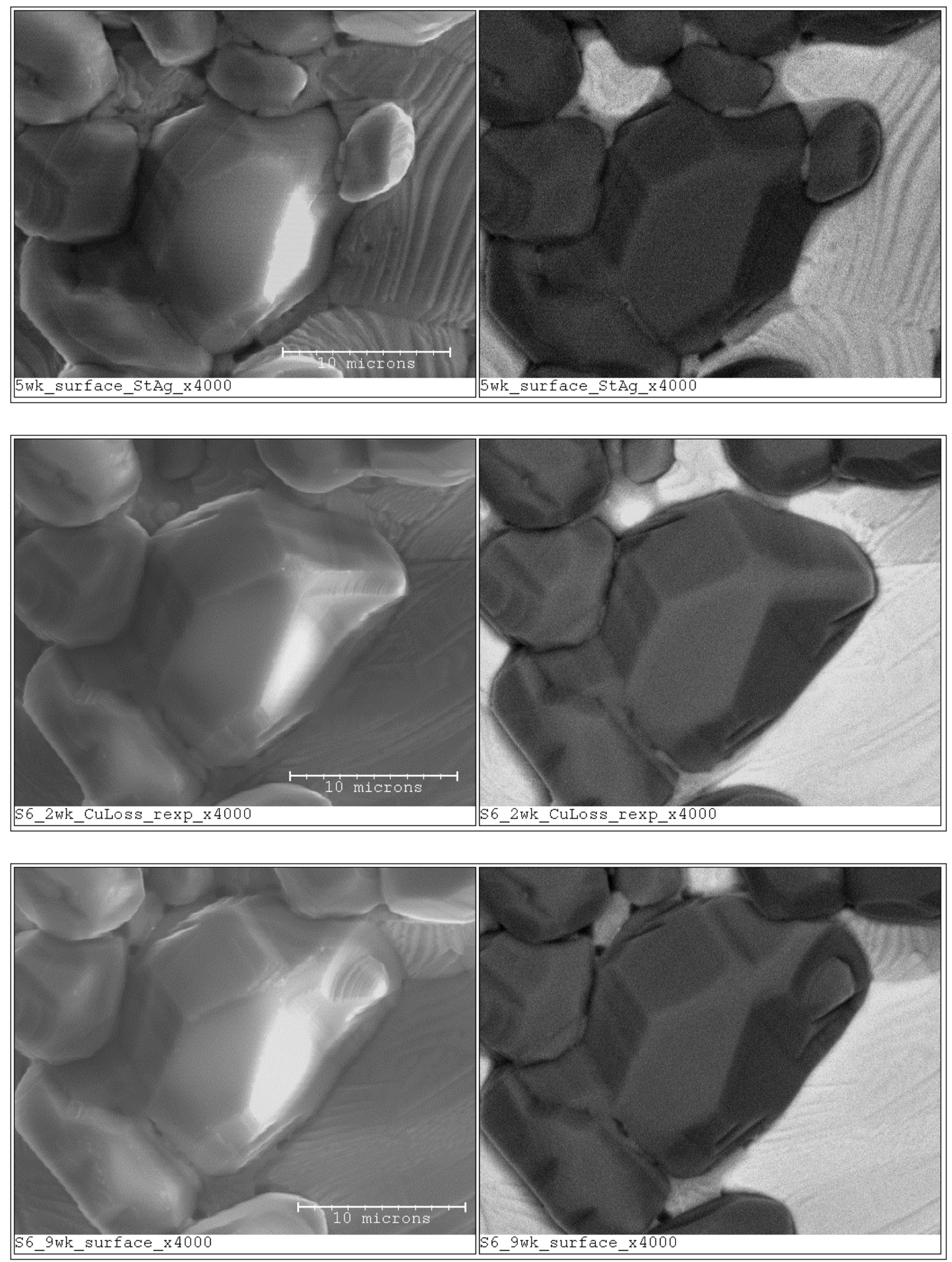

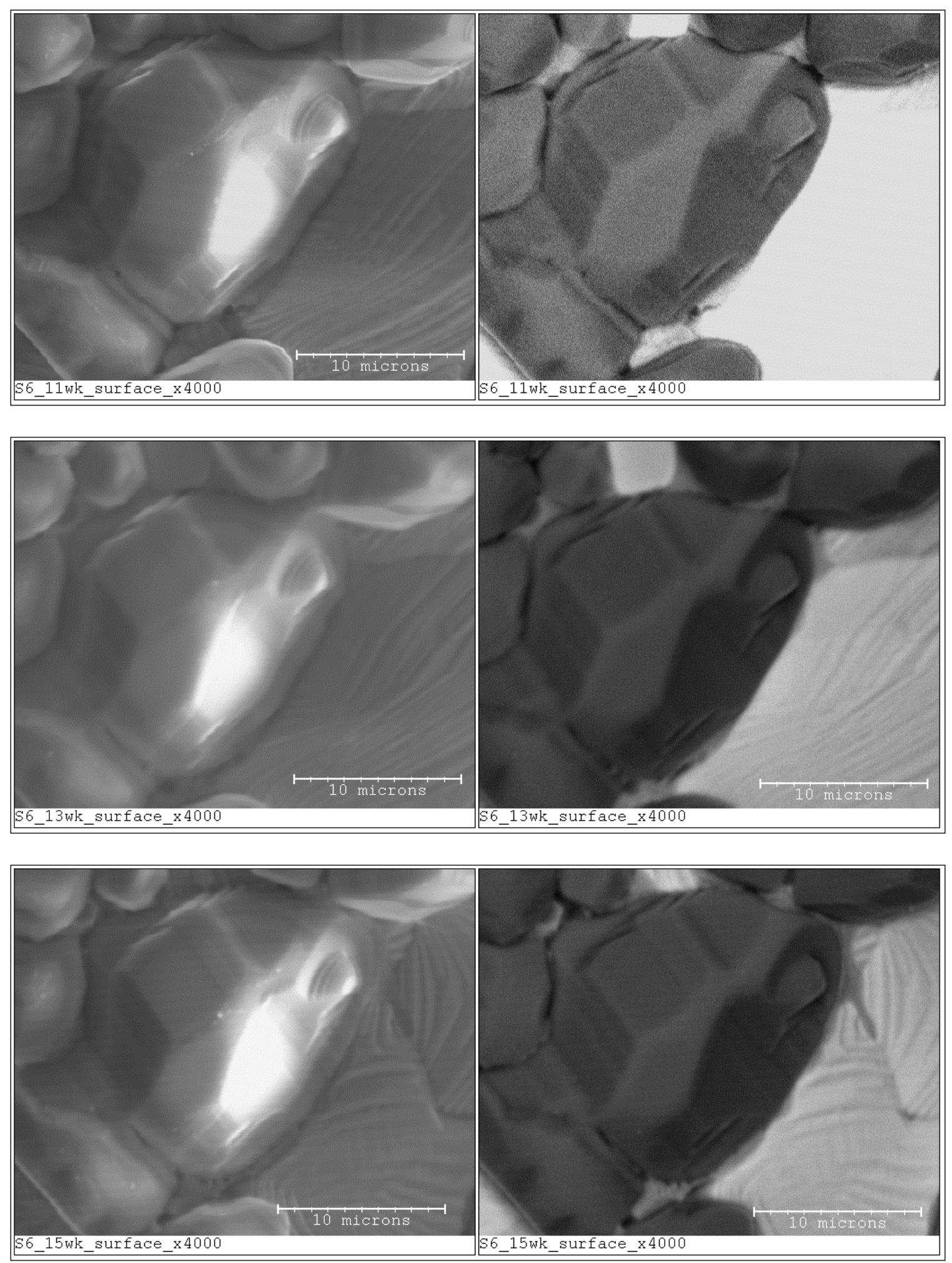

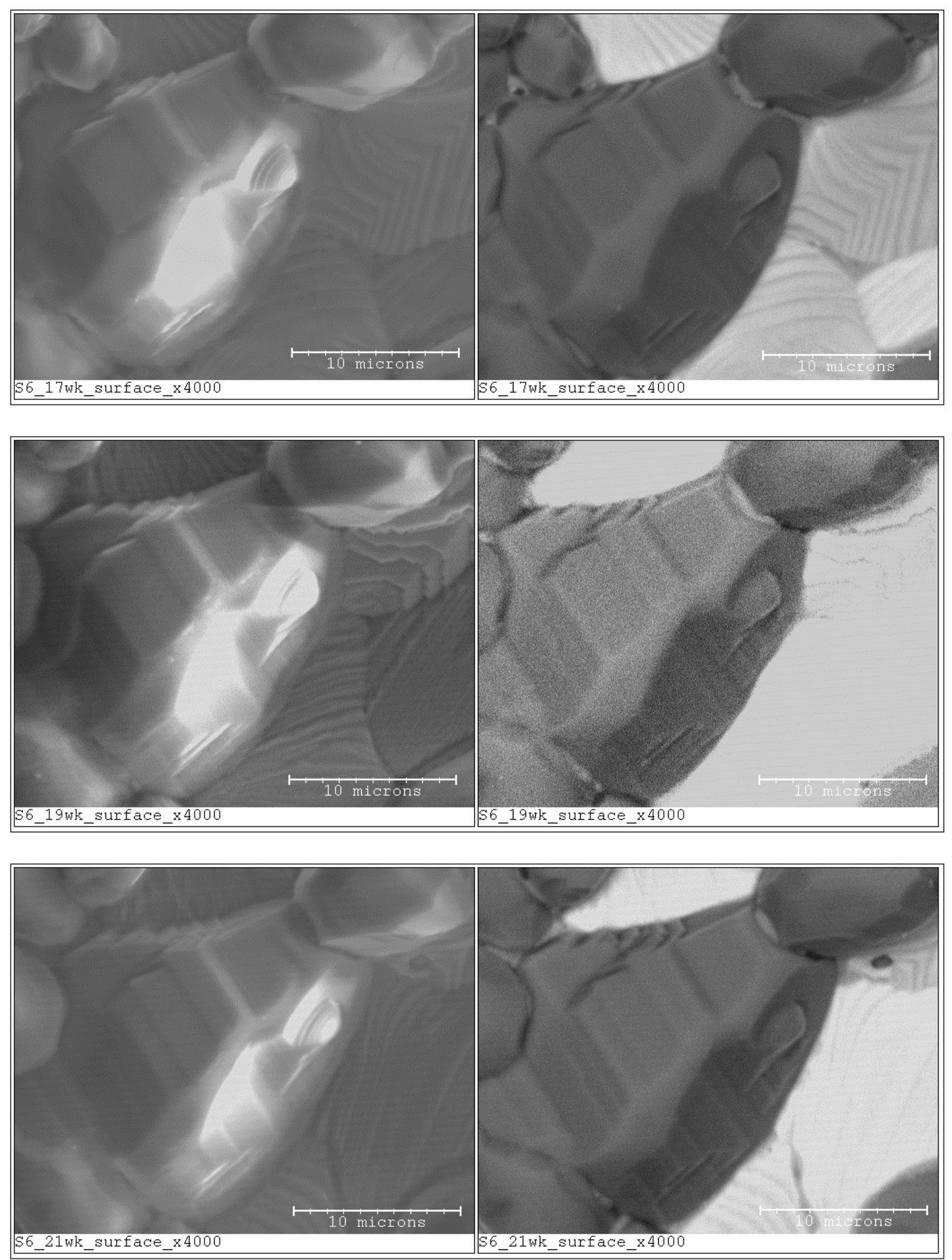
APPENDIX 5

SCANNING ELECTRON MICROSCOPY x1000 MAGNIFICATION -- TIME-

SERIES ANAYLSIS OF PRESSED SILVER/COPPER-OXIDE SAMPLE
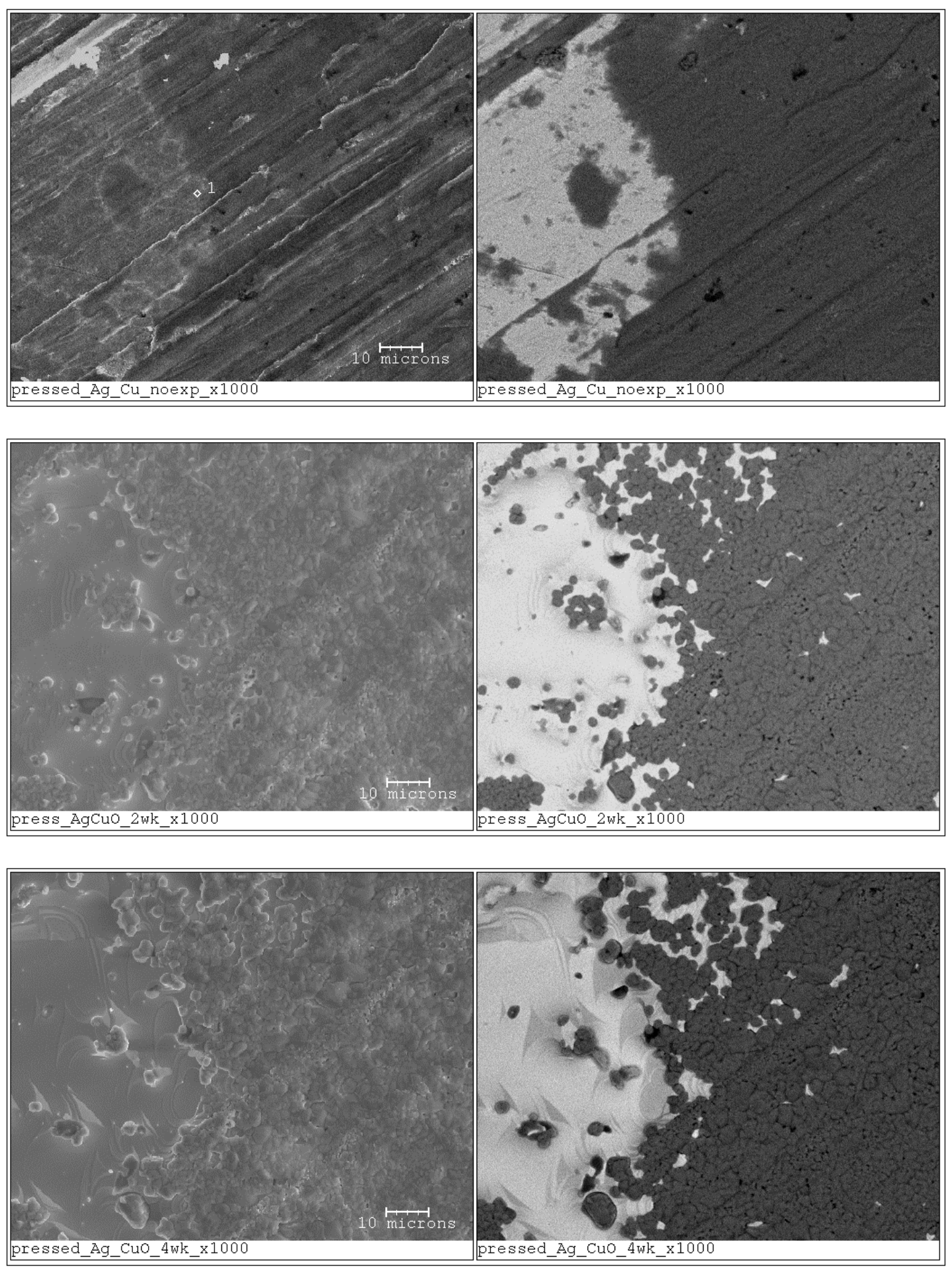

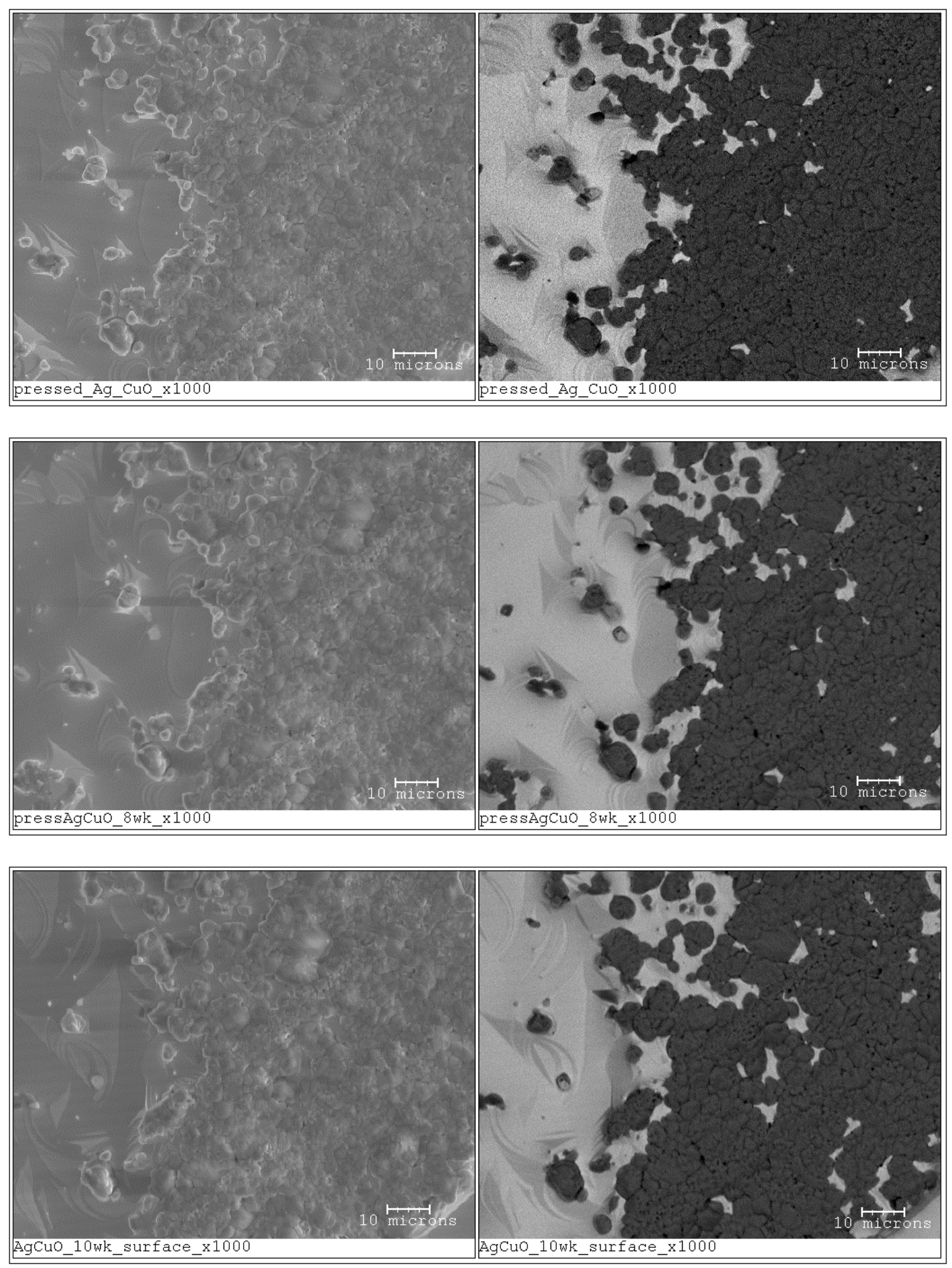

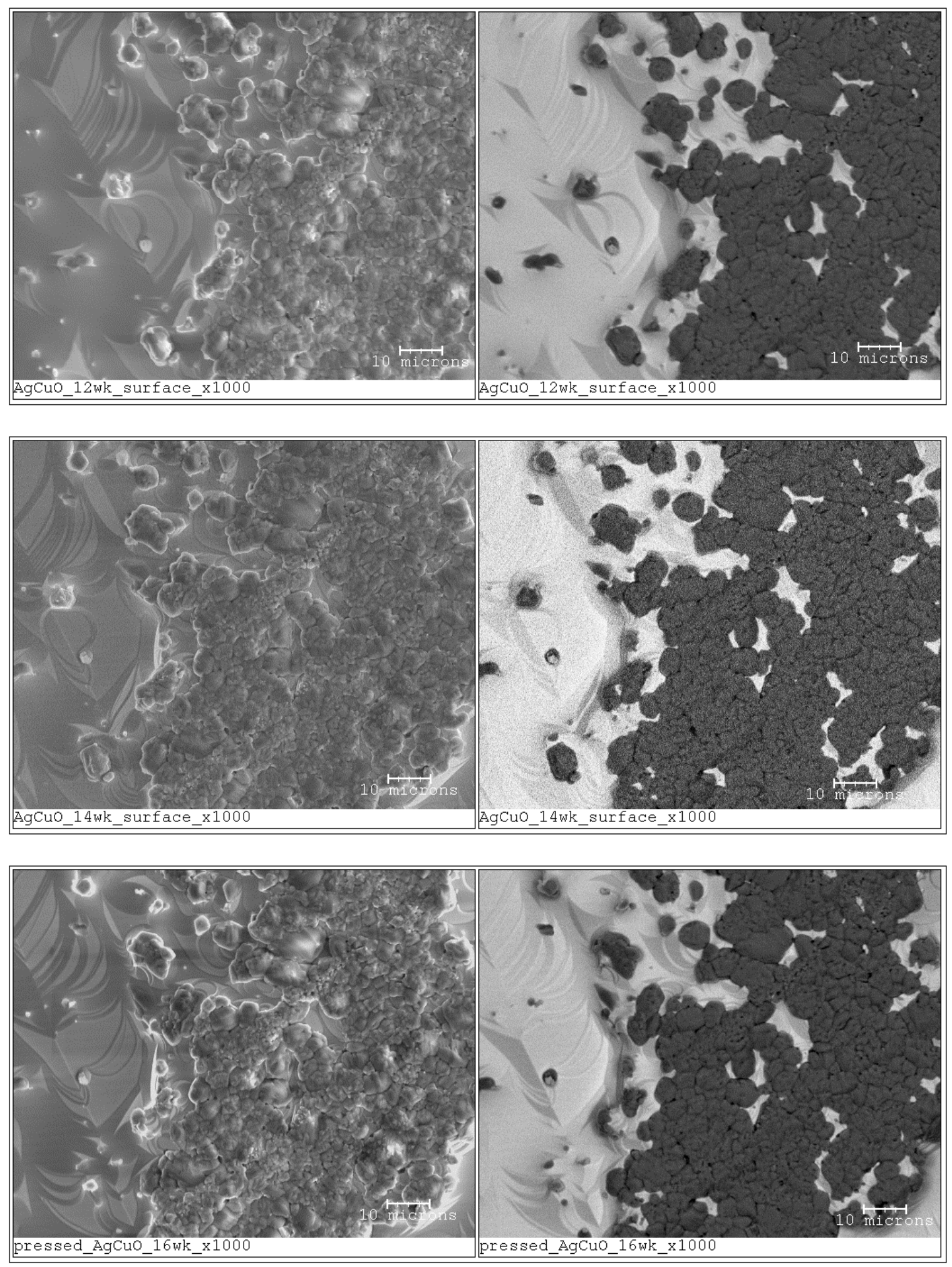


\section{APPENDIX 6}

SCANNING ELECTRON MICROSCOPY x4000 MAGNIFICATION -- TIME-

SERIES ANAYLSIS OF PRESSED SILVER/COPPER-OXIDE SAMPLE
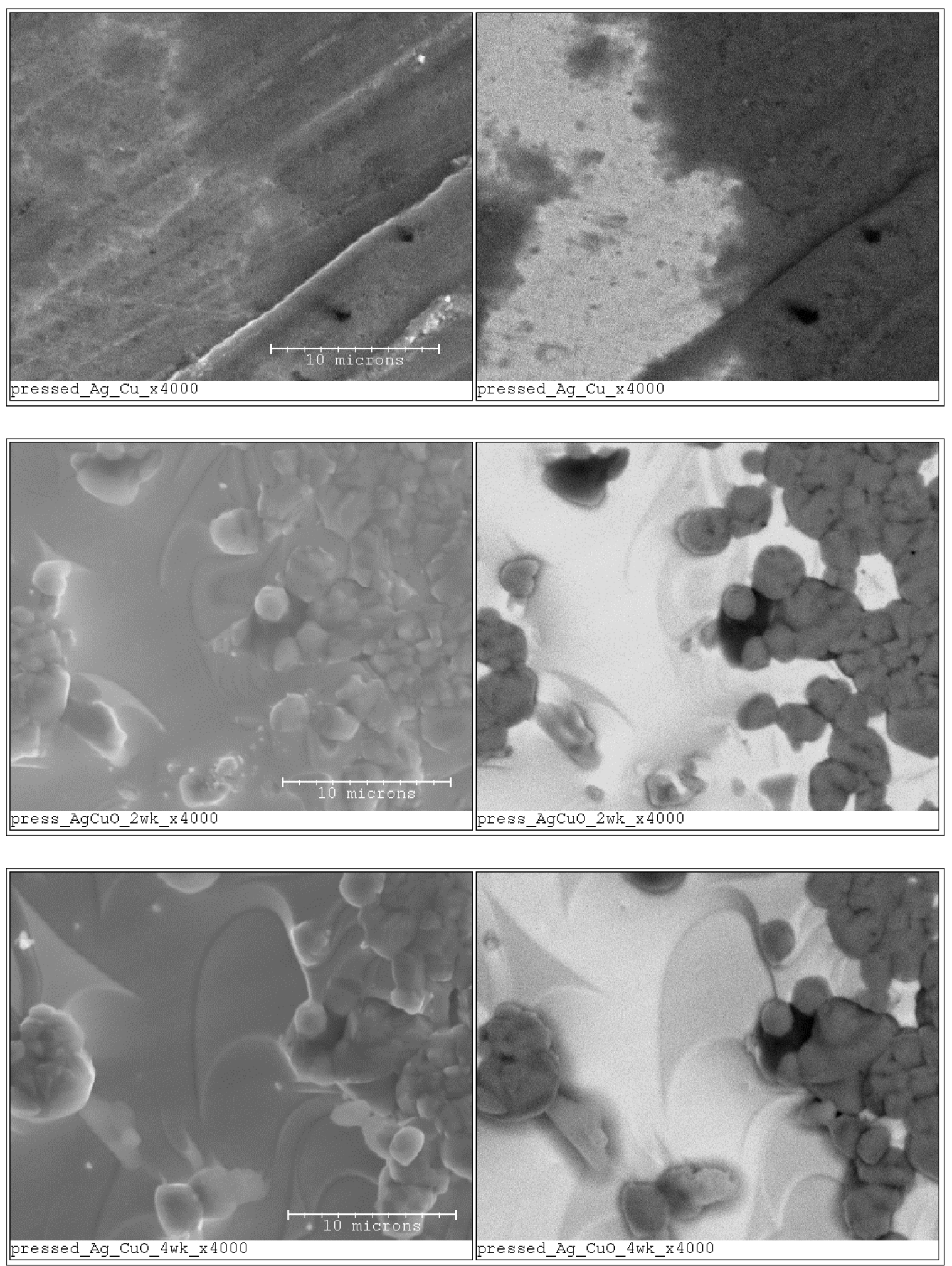

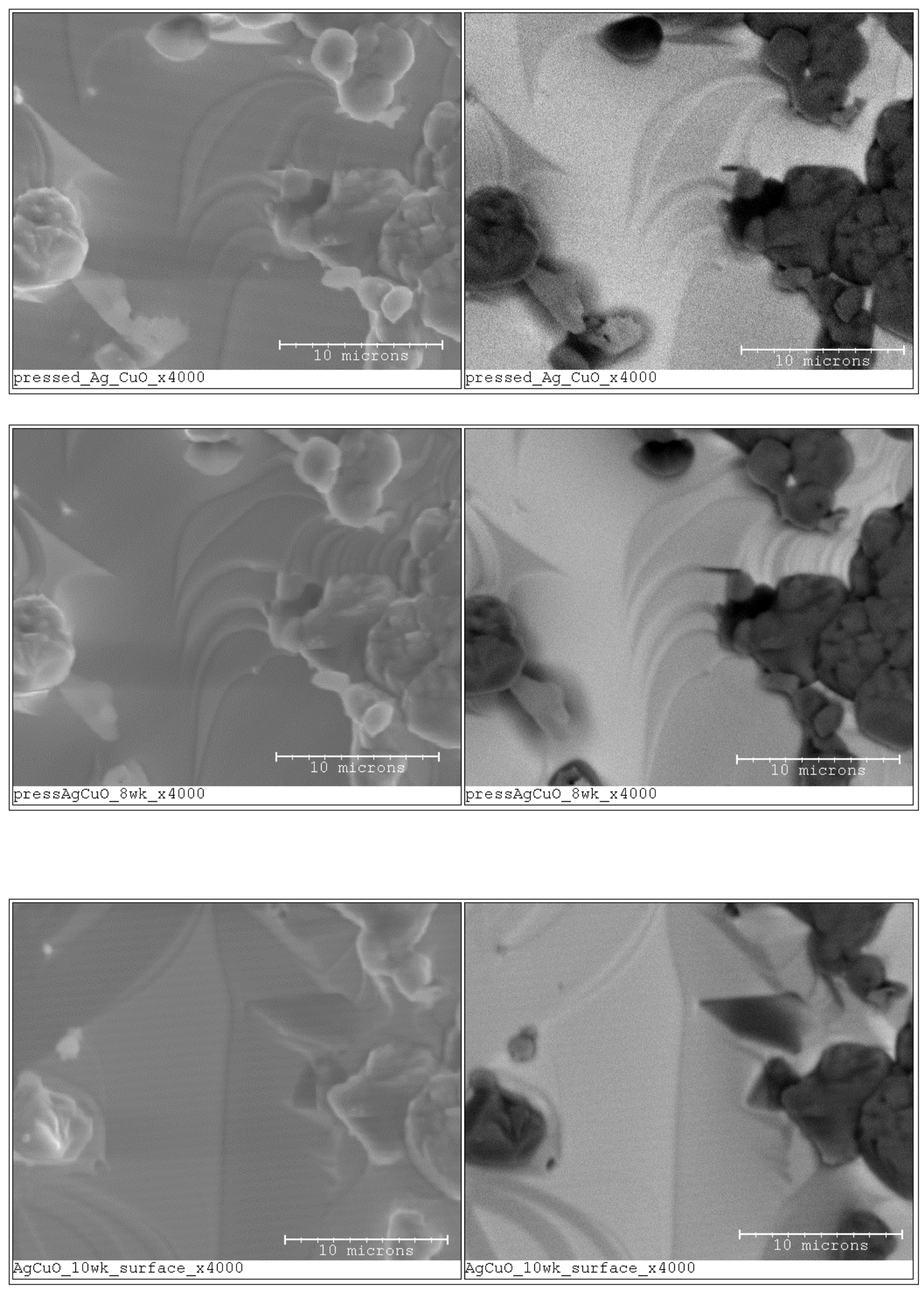

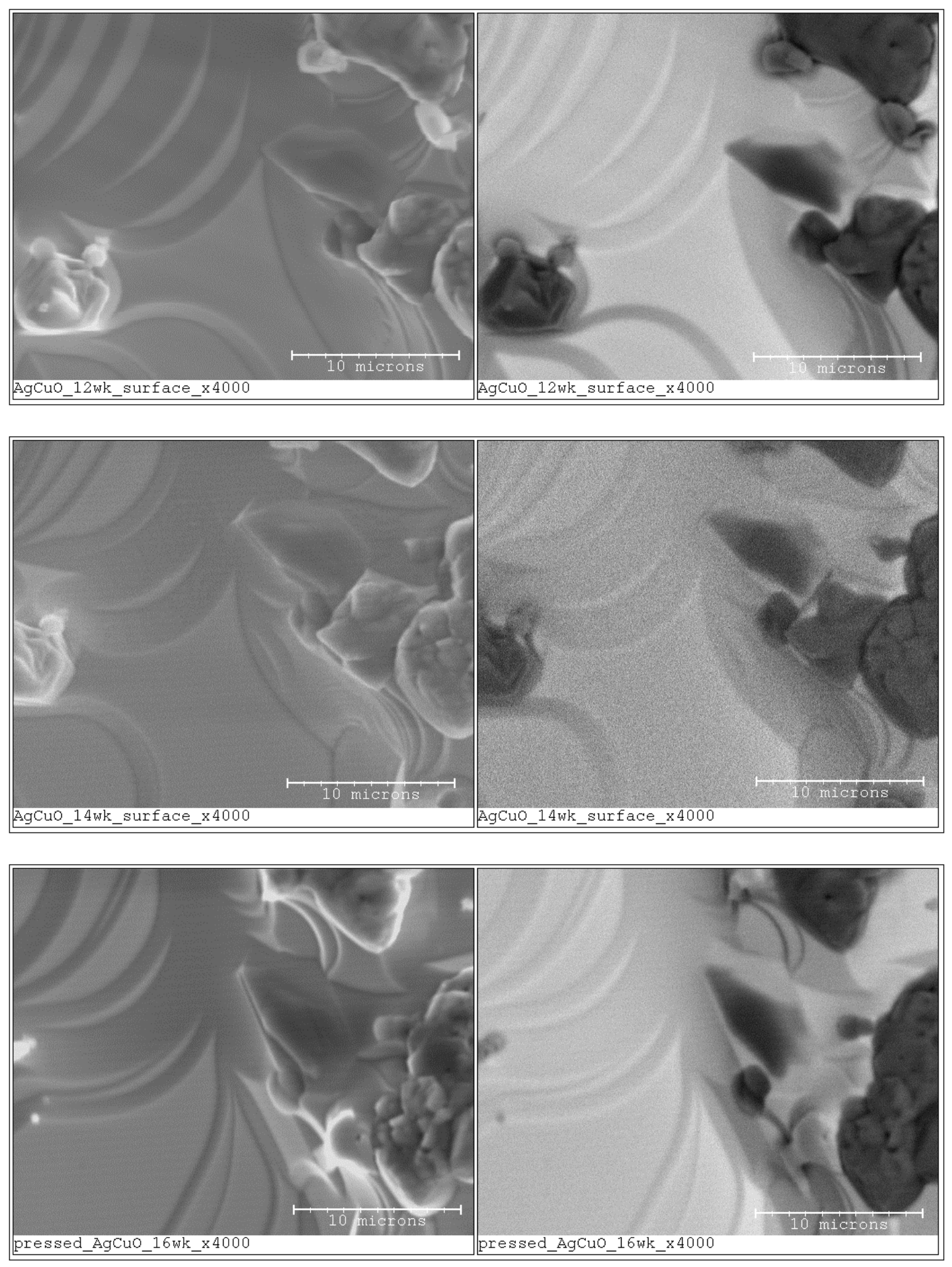
APPENDIX 7

SCANNING ELECTRON MICROSCOPY x1000 MAGNIFICATION -- TIME-

SERIES ANAYLSIS OF PRESSED SILVER/LSM SAMPLE
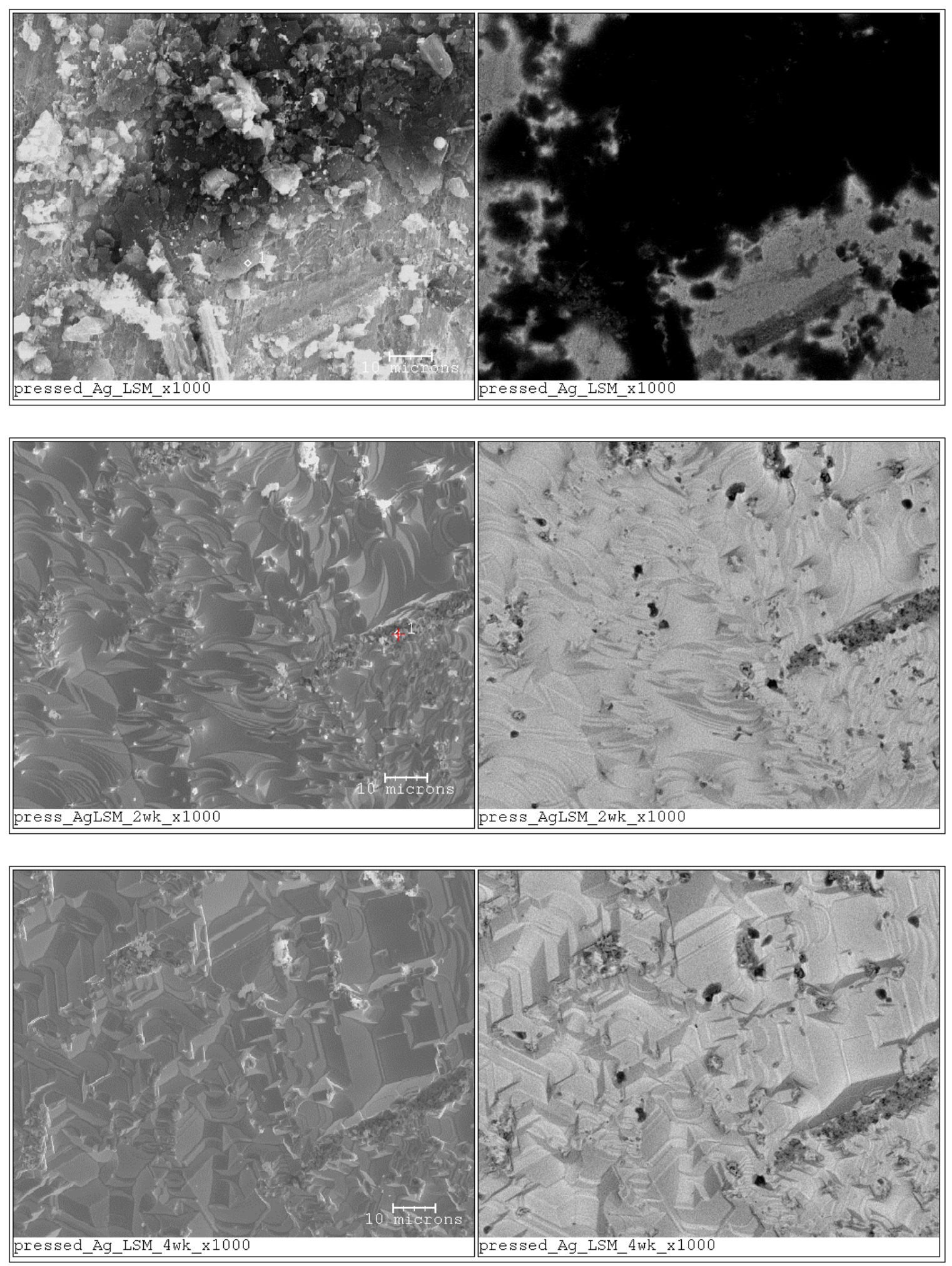

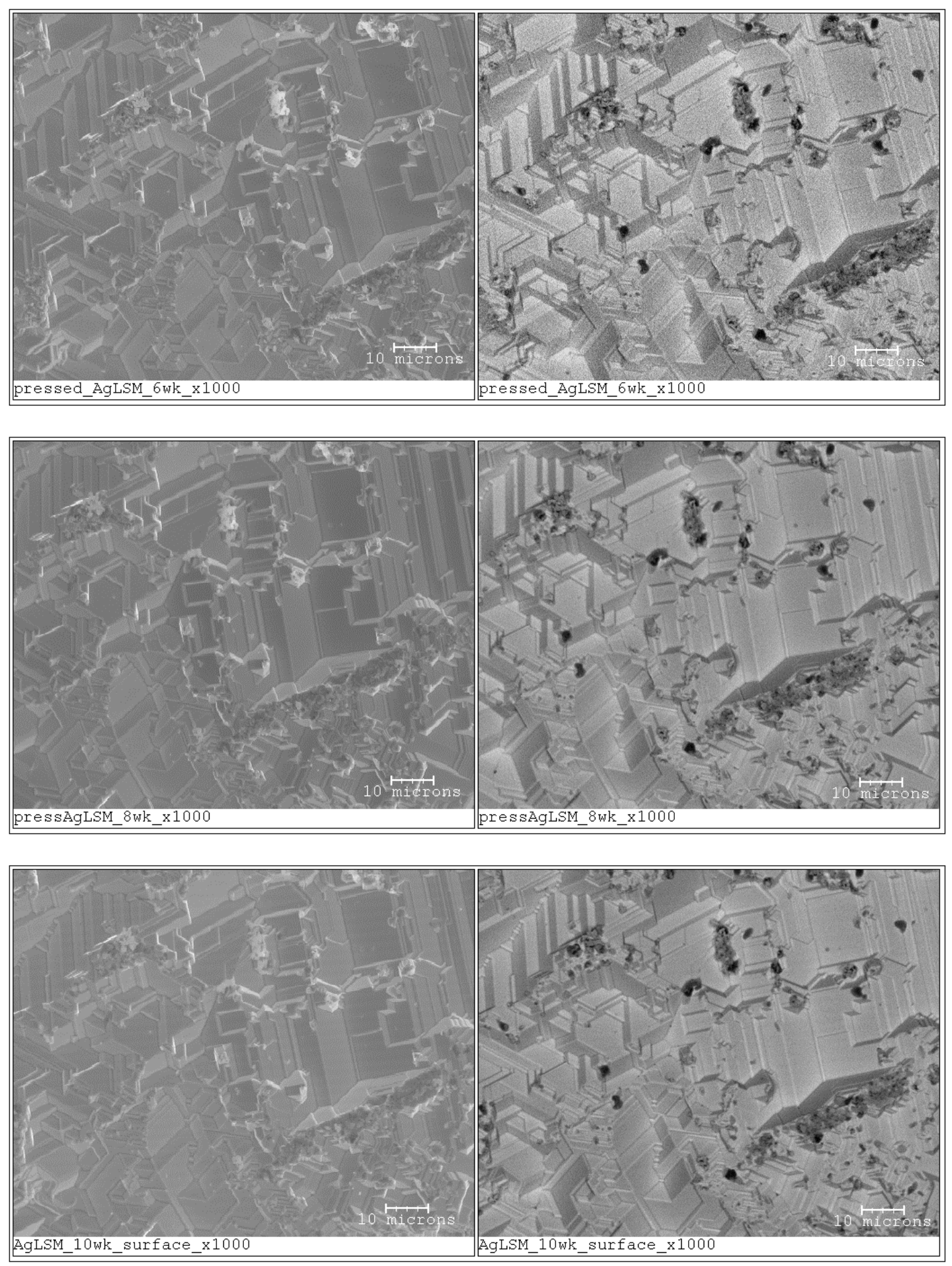

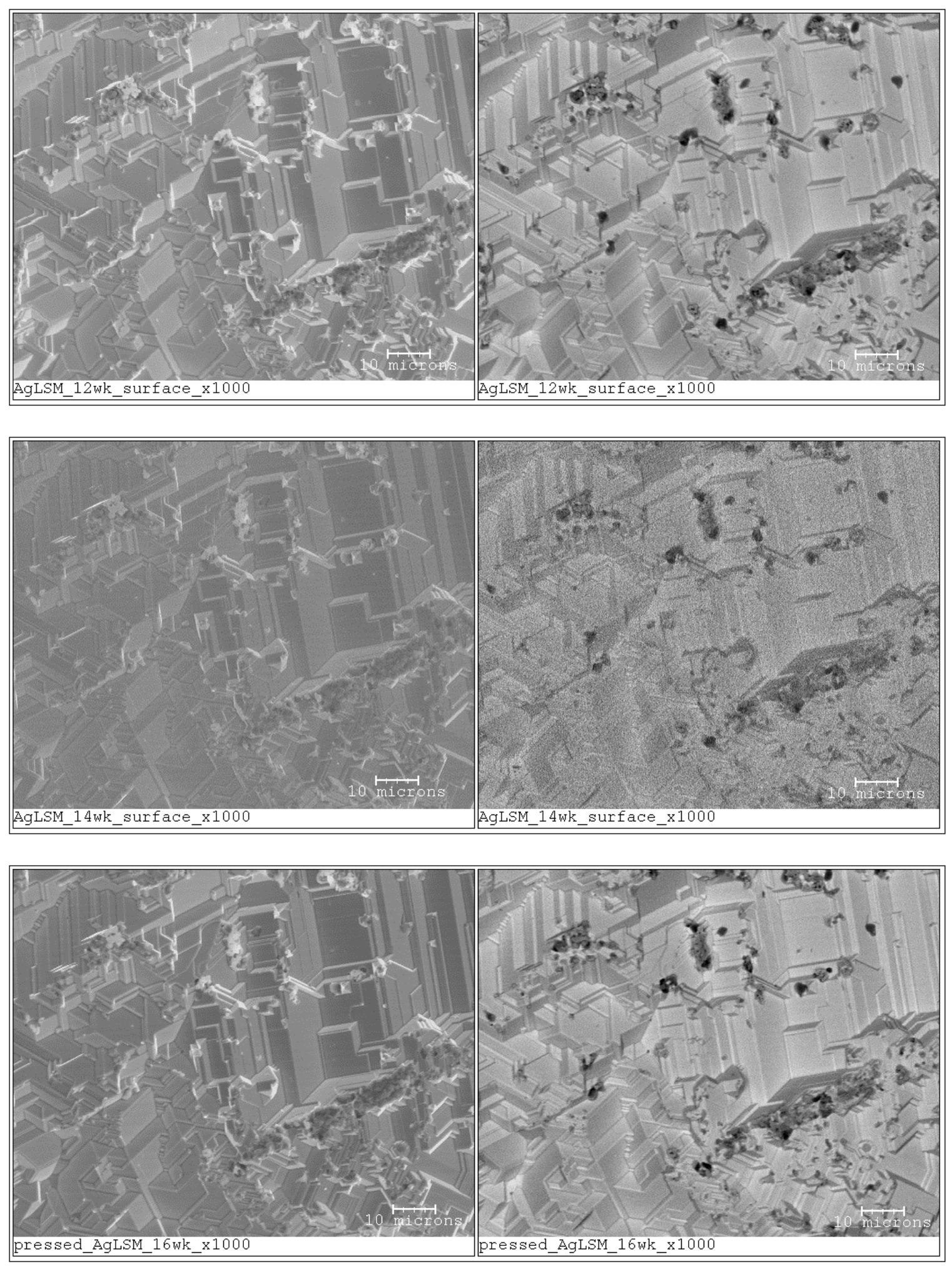
APPENDIX 8

SCANNING ELECTRON MICROSCOPY x4000 MAGNIFICATION -- TIME-

SERIES ANAYLSIS OF PRESSED SILVER/LSM SAMPLE
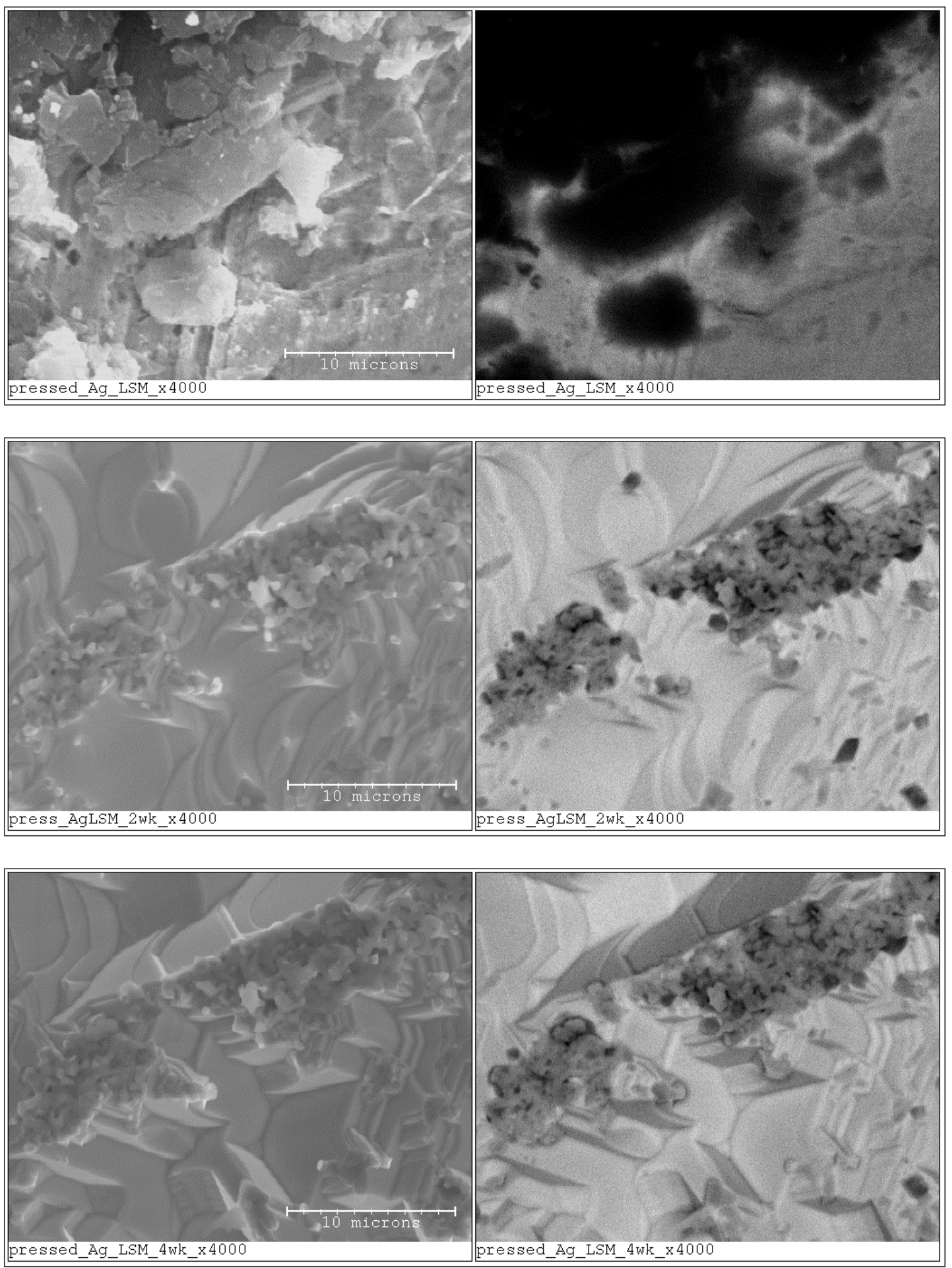

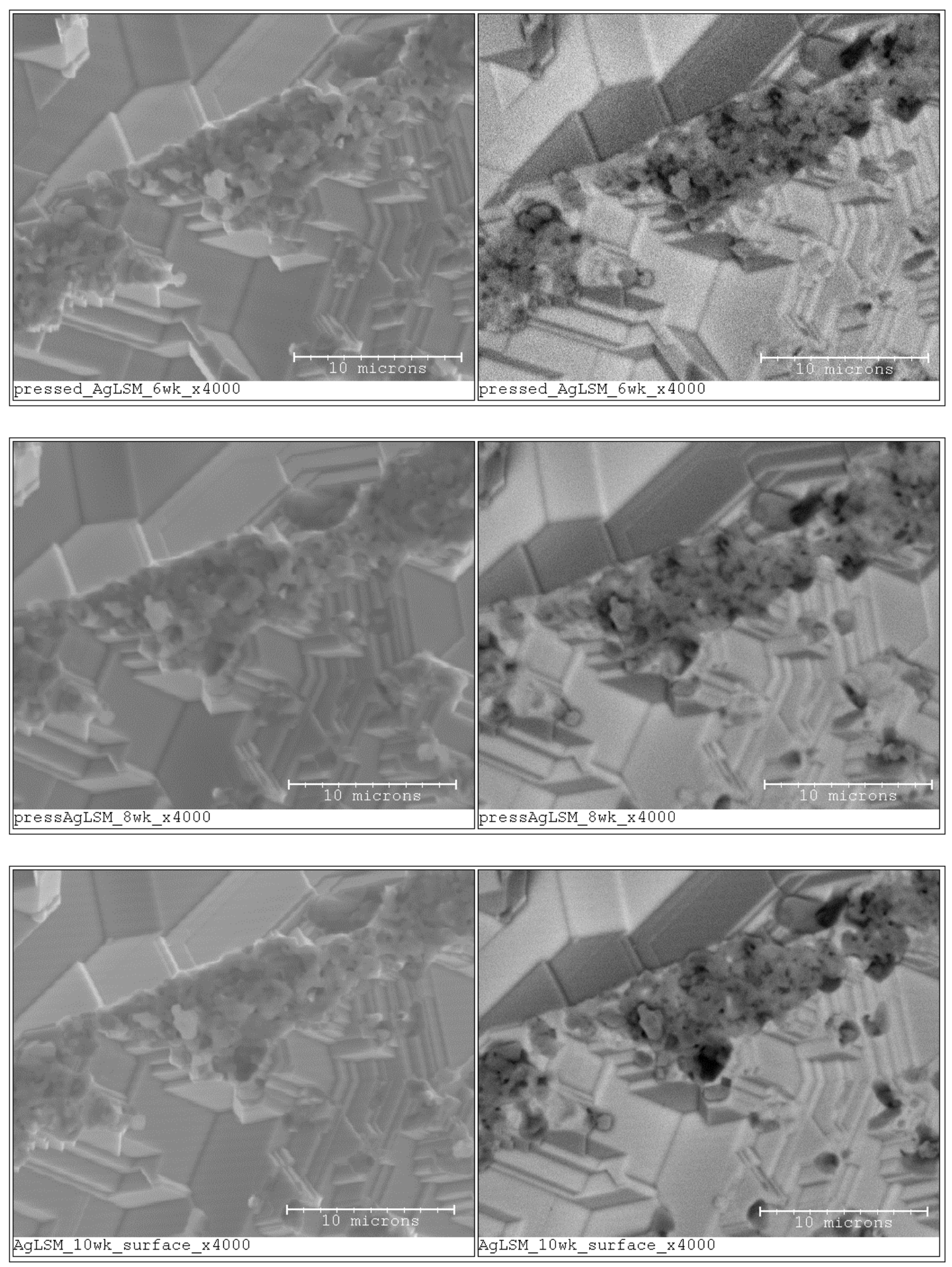

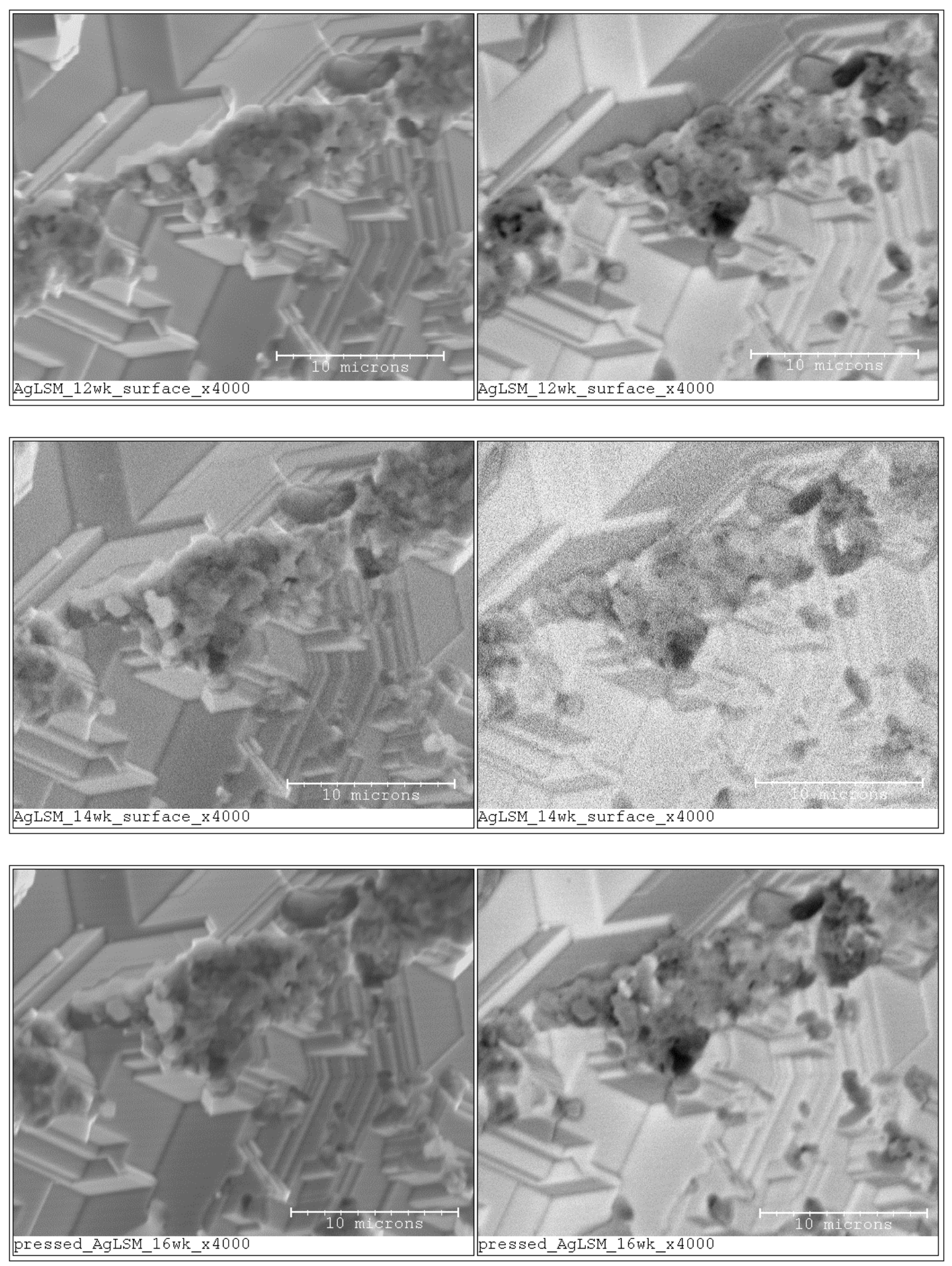


\section{APPENDIX 9}

SCANNING ELECTRON MICROSCOPY x1000 MAGNIFICATION - PURE

\section{SILVER SAMPLE SURFACE}
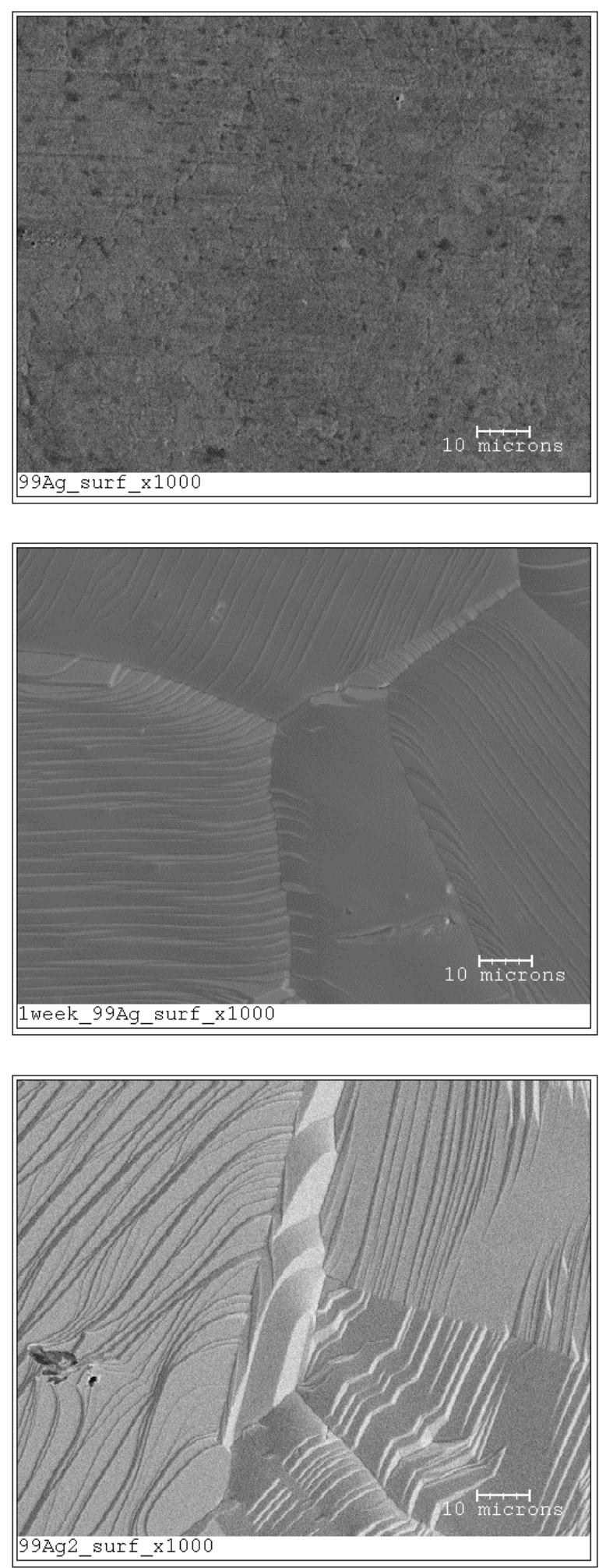

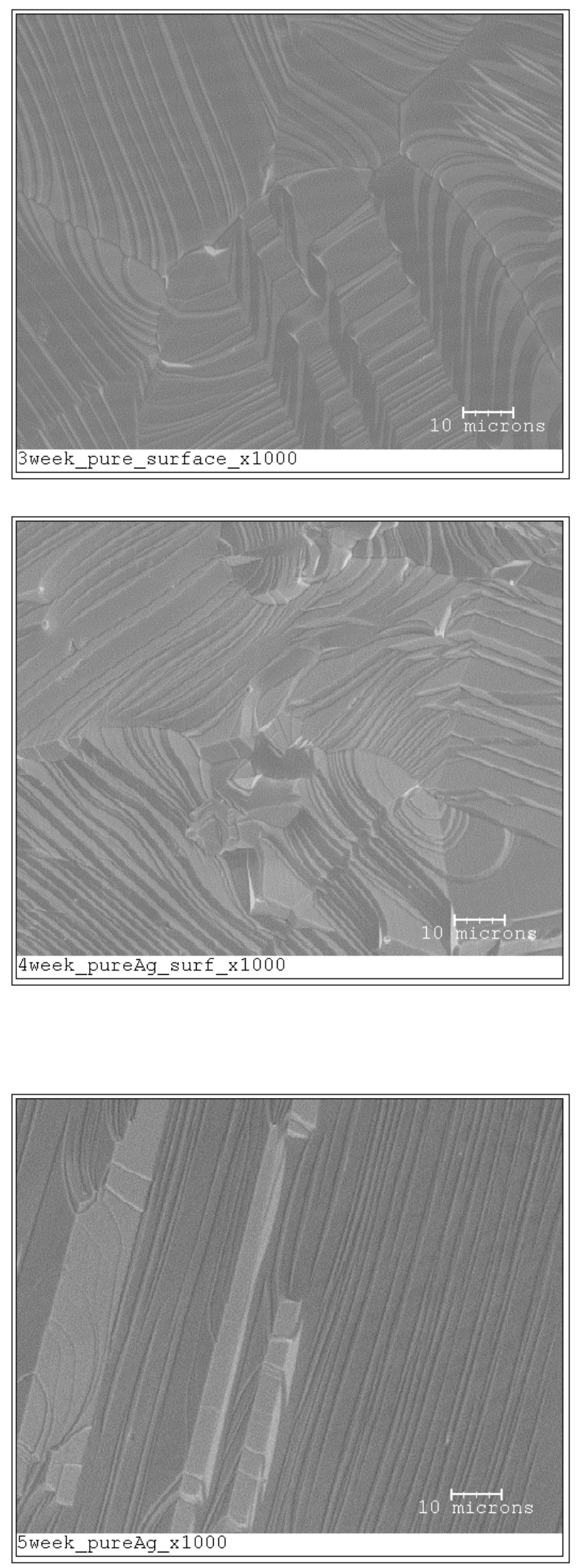

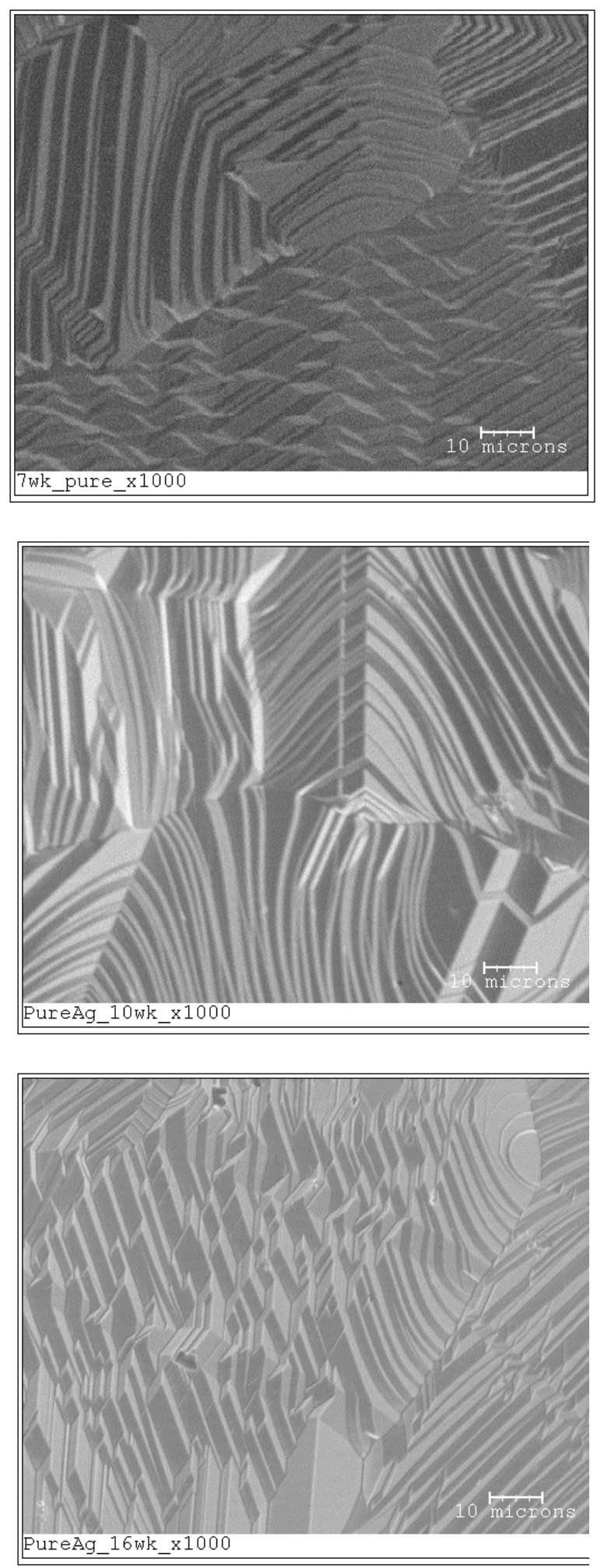


\section{REFERENCES}

1. James Larminie and Andrew Dicks; Fuel Cell Systems Explained, $2^{\text {nd }}$ Edition.

2. S.C. Singhal, Science and Technology of Solid-Oxide Fuel Cells.

3. islnotes.cse.msu.edu/trp/rtm/wet_what.html

4. Y.Niu, F. Gesmundo, F. Viani, W.Wu; The Air Oxidation of Two-Phase Cu-Ag Alloys at 650-750C; April 25, 1996; Oxidation of Metals, Vol 47, Nos. 1/2 1997.

5. B. Chalmers, R. King, R. Shuttleworth; The Thermal Etching of Silver; Proceedings of the Royal Society of London. Series A, Mathematical and Physical Sciences, Vol. 193, No. 1035. (July 21, 1948), pp. 465-483.

6. Chung-Liang Cheng, Cuo-Ciang Hsu, Ta-Jen Huang; Cathode Performance and Oxygen-ion Transport Mechanism of Copper-Oxide for Solid-Oxide Fuel Cells; Solid-State Electrochem, June 3, 2002.

7. Guangwen Zhou, Judith C. Yang; Temperature Effect on the $\mathrm{Cu}_{2} \mathrm{O}$ Oxide Morphology Created by Oxidation of $\mathrm{Cu}(001)$ as Investigated by In Situ UHV TEM; Dept. of Material Science and Engineering, University of Pittsburgh, January 27, 2003.

8. D. Majumdar, H.D. Glicksman, T.T. Kodas; Generation and Sintering Characteristics of Ag-CuO Composite Powders Made by Spray Pyrolysis;

Photopolymers and Electronic Materials, DuPont, 14 Alexander Drive, Research Triangle Park, NC 27709; Powder Technology 110(2000) 76-81.

9. Zhanguo Yang, Guanguang Xia, Jeffrey Stevenson; Electrical Contacts Between $\underline{\text { Cathodes and Metallic Interconnects in Solid-Oxide Fuel Cells; }}$ Pacific Northwest National Laboratory, 902 Battelle Blvd., Richland, WA 99352. 
10. W.L. Winterbottom; Metallurgy Department; Vaporization of Solid Silver; Ford Motor Company, Dearborn, Michigan 48121; May 7, 1969.

11. W.A. Meulenberg, O. Teller, U. Flesch, H.P. Buchkremer, D. Stover; Improved contacting by the use of silver in solid-oxide fuel cells up to an operating temperature of 800C; Journal of Material Sciences 36 (2001) 3189-3195.

12. Rahul Manepalli, Frank Stepniak, Sue Ann Bidstrup-Allen, Paul A. Kohl; $\underline{\text { Silver }}$ metallization for advanced interconnects; IEEE Transactions on Advanced Packaging, Vol. 22, No. 1, February 1999.

13. M.L. Zheludkevich, A.G. Gusakov, A.G. Vorapaev, A.A. Vecher, E.N. Kozyrski, S.A. Raspopov; Oxidation of silver by atomic oxygen; Oxidation of Metals, Vol. 61, Nos. 1/2, February 2004.

14. Geoffrey I.N. Waterhouse, Graham A. Bowmaker, James B. Metson; The thermal decomposition of silver-oxide, a combined XRD, FT-IR, and Raman spectroscopy study; Dept. of Chemistry, University of Auckland; Physical Chemistry, Chemical Physics, June 19, 2001.

15. San Ping Jiang, Siew Hwa Chan; $\underline{\text { A review of anode materials development in }}$ solid-oxide fuel cells; Fuel Cells Strategic Research Program, School of Mechanical and Production Engineering, Nanyang Technological University; Journal of Material Sciences 39 (2004) 4405-4439

16. Giovanni Dotelli, Isabella Natali Sora; Ceramic materials for SOFC: status and future prospects; Dept. of Chemistry, Material and Chemical Engineering, INSTM and Dept. of Mechanical Engineering, University of Brescia. 
17. W.Z. Zhu, S.C. Deevi; Development of interconnect materials for solid-oxide fuel cells; Research and Development Center, Chrysalis Technologies Incorportated; Material Science and Engineering; September 23, 2002; Material Science and Engineering A348(2003) 227-243.

18. W.J. Quadakkers, J. Piron-Abellan, V. Shemet, L. Singheiser; $\underline{\text { Metallic }}$ interconnectors for solid-oxide fuel cells - a review; Forschungszentrum, Julich, Germany; July 14, 2003.

19. Michael Krumpelt, James Ralph, Terry Cruse, and Joong-Myeon Bae; Materials for low-temperature solid-oxide fuel cells; Argonne National Laboratory, University of Chicago, July 5, 2002.

20. A.J. McEvoy; Materials for high-temperature oxygen reduction in solid-oxide fuel cells; Laboratory for Photonics and Interfaces, Dept. of Chemistry, Ecole Polytechnique Federale de Lausanne, Switzerland; Journal of Material Sciences 36(2001) 1087-1091.

21. Emilia Satoshi Miyamaru Seo, Walter Kenji Yoshito, Valter Ussui, Dolores Ribeiro Ricci Lazar, Sonia Regina Homem de Mello Castanho, Jose Octavio Armani Paschoal; Influence of the starting materials on performance of hightemperature oxide fuel cell devices; IPEN of San Paulo; Materials Research, Vol. 7, No. 1, 215-220, 2004. 